\begin{tabular}{|l|l} 
NCCN & $\begin{array}{l}\text { National } \\
\text { Comprehensive } \\
\text { Cancer } \\
\text { Network }^{\circledR}\end{array}$ \\
\end{tabular}

NCCN Clinical Practice Guidelines in Oncology ${ }^{\mathrm{TM}}$

\title{
Head and Neck Cancers
}

V.2.2008

Continue

www.nccn.org 


\section{NCCN Head and Neck Cancers Panel Members}

* Arlene A. Forastiere, MD/Chair †

The Sidney Kimmel Comprehensive

Cancer Center at Johns Hopkins

Kie-Kian Ang, MD, PhD §

The University of Texas M. D. Anderson

Cancer Center

David Brizel, MD §

Duke Comprehensive Cancer Center

Bruce E. Brockstein, MD † P

Robert H. Lurie Comprehensive Cancer

Center of Northwestern University

Barbara A. Burtness, MD †

Fox Chase Cancer Center

Anthony J. Cmelak, MD §

Vanderbilt-Ingram Cancer Center

Alexander D. Colevas, MD †

Stanford Comprehensive Cancer Center

Frank Dunphy, MD †

Duke Comprehensive Cancer Center

David W. Eisele, MD I

UCSF Helen Diller Family Comprehensive

Cancer Center

Helmuth Goepfert, MD $\llbracket \zeta$

The University of Texas M. D. Anderson

Cancer Center

Wesley L. Hicks, Jr., MD đ

Roswell Park Cancer Institute

Merrill S. Kies, MD †

The University of Texas M. D. Anderson

Cancer Center
William M. Lydiatt, MD $\ \zeta$

UNMC Eppley Cancer Center at The Nebraska

Medical Center

Ellie Maghami, MD \ $\zeta$

City of Hope

Renato Martins, MD, MPH †

Fred Hutchinson Cancer Research Centerl

Seattle Cancer Care Alliance

Thomas McCaffrey, MD, PhD $\zeta$

H. Lee Moffitt Cancer Center \& Research Institute

Bharat B. Mittal, MD $\S$

Robert H. Lurie Comprehensive Cancer Center of Northwestern University

David G. Pfister, MD † P

Memorial Sloan-Kettering Cancer Center

Harlan A. Pinto, MD † P

Stanford Comprehensive Cancer Center

Marshall R. Posner, MD † $P$

Dana-Farber/Brigham and Women's Cancer

Center | Massachusetts General Hospital Cancer

Center

John A. Ridge, MD, PhD ๆ

Fox Chase Cancer Center

Sandeep Samant, MD I

St. Jude Children's Research Hospital/University of Tennessee Cancer Institute

\section{Continue}

David E. Schuller, MD I

Arthur G. James Cancer Hospital \&

Richard J. Solove Research Institute at

The Ohio State University

Jatin P. Shah, MD ף

Memorial Sloan-Kettering Cancer Center

Sharon Spencer, MD §

University of Alabama at Birmingham

Comprehensive Cancer Center

Andy Trotti, III, MD §

H. Lee Moffitt Cancer Center \& Research Institute

Randal S. Weber, MD

The University of Texas M. D. Anderson

Cancer Center

Gregory T. Wolf, MD \ $\zeta$

University of Michigan Comprehensive

Cancer Center

Frank Worden, MD I †

University of Michigan Comprehensive

Cancer Center

† Medical Oncology

Tा Surgery/Surgical oncology

$\S$ Radiation oncology/Radiotherapy

$\zeta$ Otolaryngology

P Internal medicine

* Writing Committee Member 


\section{Table of Contents}

NCCN Head and Neck Cancers Panel Members

Summary of Guidelines Updates

- Multidisciplinary Team Approach (TEAM-1)

- Support Modalities (TEAM-1)

- Ethmoid Sinus Tumors (ETHM-1)

- Maxillary Sinus Tumors (MAXI-1)

- Salivary Gland Tumors (SALI-1)

- Cancer of the Lip (LIP-1)

- Cancer of the Oral Cavity (OR-1)

- Cancer of the Oropharynx (ORPH-1)

- Cancer of the Hypopharynx (HYPO-1)

- Occult Primary (OCC-1)

- Cancer of the Glottic Larynx (GLOT-1)

- Cancer of the Supraglottic Larynx (N0) (SUPRA-1)

- Cancer of the Nasopharynx (NASO-1)

- Unresectable Head and Neck Cancer (ADV-1)

- Recurrent Head and Neck Cancer (ADV-2)

- Principles of Systemic Therapy (CHEM-A)

For help using these

documents, please click here

Staging

Manuscript

References

Clinical Trials: The NCCN

believes that the best management for any cancer patient is in a clinical trial. Participation in clinical trials is especially encouraged.

To find clinical trials online at NCCN member institutions, click here: nccn.org/clinical_trials/physician.html

NCCN Categories of Evidence and Consensus: All recommendations are Category $2 \mathrm{~A}$ unless otherwise specified.

See NCCN Categories of Evidence and Consensus

Guidelines Index

Print the Head and Neck Cancers Guideline

These guidelines are a statement of evidence and consensus of the authors regarding their views of currently accepted approaches to treatment. Any clinician seeking to apply or consult these guidelines is expected to use independent medical judgment in the context of individual clinical circumstances to determine any patient's care or treatment. The National Comprehensive Cancer Network makes no representations or warranties of any kind, regarding their content use or application and disclaims any responsibility for their application or use in any way. These guidelines are copyrighted by National Comprehensive Cancer Network. All rights reserved. These guidelines and the illustrations herein may not be reproduced in any form without the express written permission of NCCN. C2008. 


\section{Summary of the Guidelines updates}

The Guidelines were updated to version 2.2008 to represent the addition of the manuscript correspondent to the changes in the algorithm.

Summary of changes in the 1.2008 version of the Head and Neck Cancer guidelines from the 1.2007 version include:

Global Changes

- In the workup sections, chest $\mathrm{x}$-ray and/or chest CT was changed to chest imaging.

- The indication for postoperative chemoradiation was changed from "one or both major risk features or $\geq 2$ minor risk features" to

"extracapsular spread and/or positive margins".

- In the follow-up sections "Smoking cessation counseling" was added. In sections that include "Speech and swallowing evaluation",

"hearing" was also added. "Dental evaluation" was added to some of the sites. For advanced disease "Post treatment baseline imaging of the primary and neck was added with the additional recommendation to "reimage as indicated based upon signs/symptoms on physical exam."

TEAM-1

- Multidisciplinary Team

Audiology was added to Adjunctive Services.

- Support and Services

The following were added: General medical care, Smoking and "alcohol" cessation, Wound management, Depression assessment and management.

Ethmoid Sinus Tumors

ETHM-1

- Small cell neuroendocrine was added to the malignant pathology listing for Ethmoid Sinus tumors.

- Footnote 'a' is new to the page emphasizing systemic therapy for some histologic subtypes.

ETHM-2

- Footnote ' $c$ ' - perineural invasion was changed to intracranial extension.

Maxillary Sinus Tumors

MAXI-1

- Footnote ' $b$ ' is new to the page emphasizing systemic therapy for some histologic subtypes.

Salivary Gland Tumors

SALI-3

- For tumors that are completely excised, adenoid cystic was given its own treatment pathway with the recommendation of "adjuvant RT" and a category $2 \mathrm{~B}$ recommendation. 


\section{Summary of the Guidelines updates}

Summary of changes in the 1.2008 version of the Head and Neck Cancer guidelines from the 1.2007 version include:

Cancer of the Lip

LIP-2

- The recommendation for chemo/RT was removed as an option in adjuvant treatment.

ㄴIP-3

- Chemo/RT was added as a primary treatment option.

Cancer of the Oral Cavity

OR-2 and OR-3

- The recommendation to consider chemo/RT was added as an option in adjuvant treatment for patient with other risk features.

Cancer of the Oropharynx

ORPH-1

- HPV testing was added to the workup section.

ORPH-2 and ORPH-3

- The recommendation to consider chemo/RT was added as an option in adjuvant treatment for patient with other risk features.

Cancer of the Hypopharynx

HYPO-2, HYPO-3 and HYPO-4

- The recommendation to consider chemo/RT was added as an option in adjuvant treatment for patient with other risk features.

Occult Primary

OCC-1

- HPV, EBV testing was added to the workup section for squamous cell or undifferentiated histology.

OCC-2

- Adenocarcinoma levels IV and V has its own pathway with the recommendation of selective neck dissection and then evaluate for infraclavicular primary.

- Induction chemotherapy followed by chemo/RT or RT was added as a primary treatment option with a category 3 designation.

Cancer of the Glottic Larynx

GLOT-3

- The recommendation to consider chemo/RT was added as an option in adjuvant treatment for patient with other risk features.

Cancer of the Supraglottic Larynx

SUPRA-3, SUPRA-6 and SUPRA-7

- The recommendation to consider chemo/RT was added as an option in adjuvant treatment for patient with other risk features.

Recurrent Head and Neck Cancer

ADV-2

- The recommendation for chemo/RT was added as a treatment option for resectable patients with locoregional recurrence and no prior RT. 


\begin{tabular}{|c|c|}
\hline \multicolumn{2}{|c|}{ MULTIDISCIPLINARY TEAM } \\
\hline \multicolumn{2}{|c|}{$\begin{array}{l}\text { The management of patients with head and neck cancers is complex. All patients need } \\
\text { access to the full range of specialists and support services with expertise in the } \\
\text { management of patients with head and neck cancer for optimal treatment and follow-up }\end{array}$} \\
\hline $\begin{array}{l}\text { - Head and neck surgery } \\
\text { - Radiation oncology } \\
\text { - Medical oncology } \\
\text { - Plastic and reconstructive surgery } \\
\text { - Specialized nursing care } \\
\text { - Dentistry/prosthodontics } \\
\text { - Physical medicine and } \\
\text { rehabilitation } \\
\text { - Speech and swallowing therapy }\end{array}$ & $\begin{array}{l}\text { - Clinical Social work } \\
\text { - Nutrition support } \\
\text { - Pathology } \\
\text { - Diagnostic radiology } \\
\text { - Adjunctive services } \\
\text { - Neurosurgery } \\
\text { - Ophthalmology } \\
\text { - Psychiatry } \\
\text { - Addiction Services } \\
\text { - Audiology }\end{array}$ \\
\hline
\end{tabular}

\section{SUPPORT AND SERVICES}

Follow-up should be performed by a physician with expertise in the management and prevention of treatment sequelae. It should include a comprehensive head and neck exam. The management of head and neck cancer patients may involve the following:

- General medical care

- Pain and symptom management

- Nutritional support

- Enteral feeding

- Oral supplements

- Dental care for RT effects

- Xerostomia management

- Smoking and alcohol cessation
- Tracheotomy care

- Wound management

- Depression assessment and management

- Social work and Case management

- Supportive Care (See NCCN Palliative Care Guideline) 


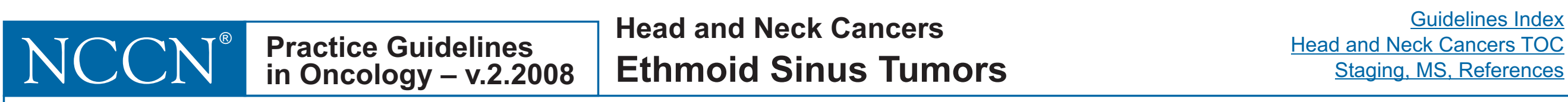

\section{WORKUP}

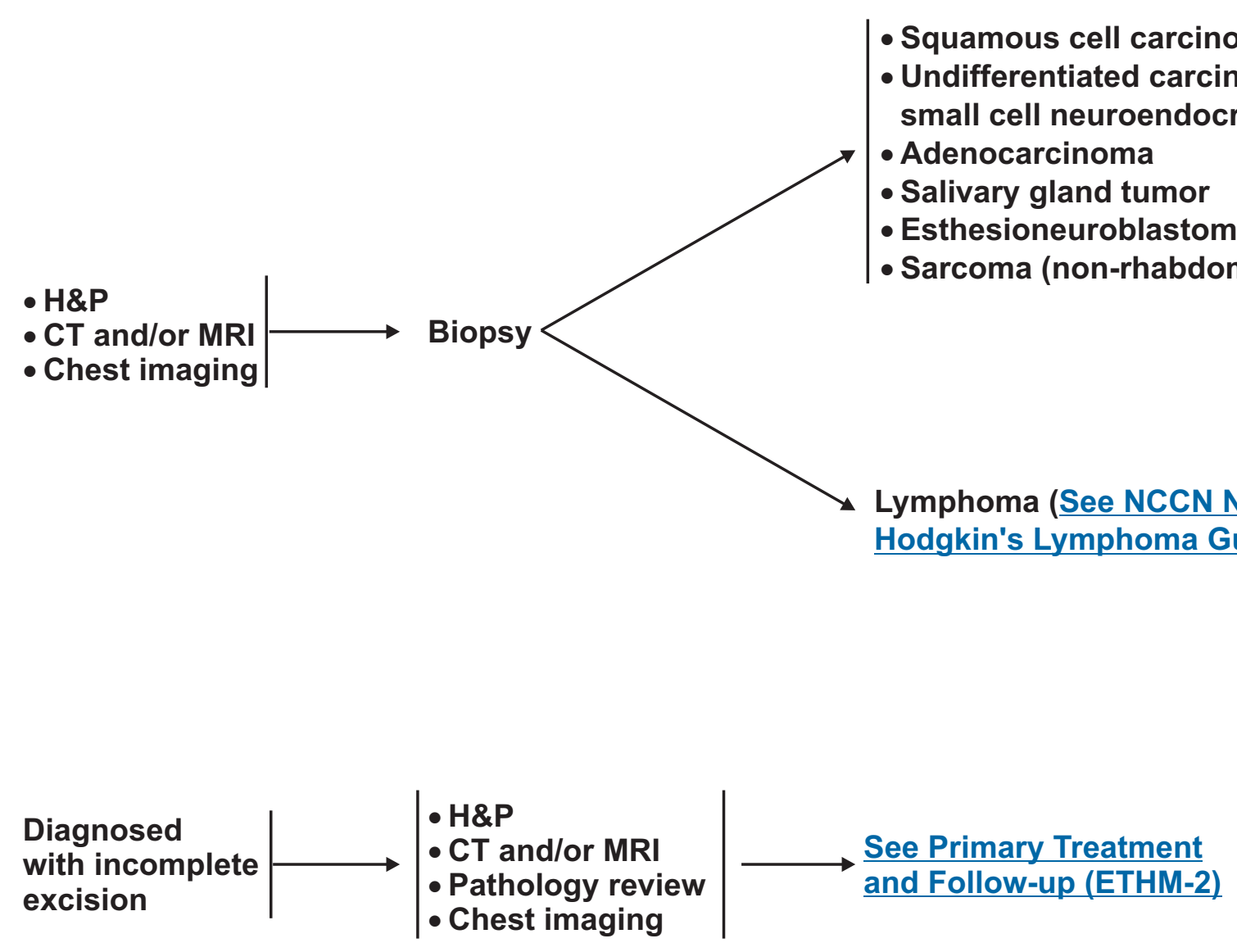

${ }^{a}$ For sinonasal undifferentiated carcinomas (SNUC), esthesioneuroblastoma, and small cell neuroendocrine histologies, systemic therapy should be a part of the overall treatment.

Note: All recommendations are category $2 \mathrm{~A}$ unless otherwise indicated.

Clinical Trials: NCCN believes that the best management of any cancer patient is in a clinical trial. Participation in clinical trials is especially encouraged. 


\section{CLINICAL PRESENTATION}

Newly diagnosed; T1, T2

Newly diagnosed; T3, T4a resectable

$\longrightarrow\left|\begin{array}{l}\text { Complete surgical } \\ \text { resection (preferred) }\end{array}\right|$

Complete

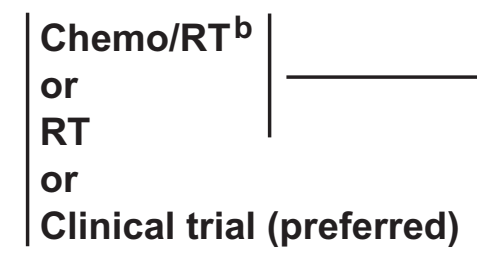

Newly diagnosed, unresectable

$\longrightarrow$

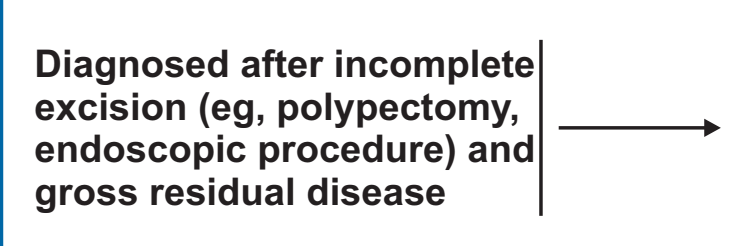

Diagnosed after incomplete exision (eg, polypectomy, endoscopic procedure) and no disease on physical exam, imaging, and/or endoscopy

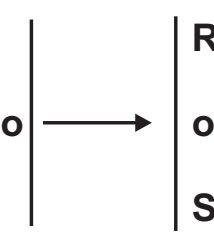
surgical resection

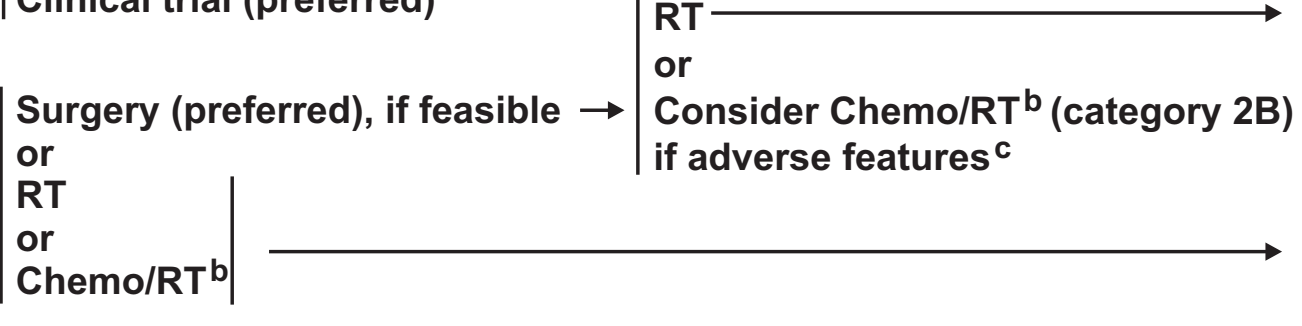

\section{ADJUVANT TREATMENT}

RT

Consider Chemo/RT ${ }^{\text {b }}$ (category 2B) if adverse features ${ }^{c}$

or

Consider Chemo/RT ${ }^{\text {b }}$ (category 2B) if adverse features ${ }^{c}$

Chemo/RT
or
RT
or

Clinical trial (preferred)

or

Surgery, if feasible

\section{FOLLOW-UP}

b See Principles of Systemic Therapy (CHEM-A).

${ }^{\mathrm{c}}$ Adverse features include positive margins and intracranial extension.

Note: All recommendations are category $2 \mathrm{~A}$ unless otherwise indicated.

Clinical Trials: NCCN believes that the best management of any cancer patient is in a clinical trial. Participation in clinical trials is especially encouraged.

- Physical exam:

Year 2,

$>5$ years,

every 6-12 mo

neck irradiated

- CT scan/MRI- baseline

(category 2B) 


\section{$\mathrm{NCCN}^{\oplus}$ Practice Guidelines

\section{WORKUP}
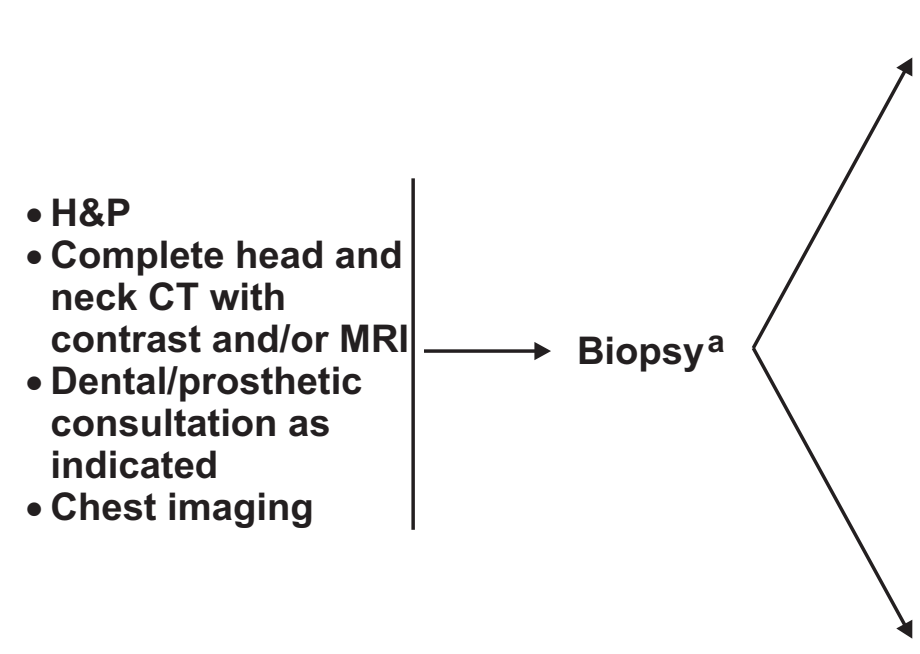

Lymphoma

\section{PATHOLOGY}

- Squamous cell carcinoma

- Undifferentiated carcinoma

(SNUC, small cell

neuroendocrine) $)^{b}$

- Adenocarcinoma

- Salivary gland tumor

- Esthesioneuroblastoma ${ }^{b}$

- Sarcoma (non-

rhabdomyosarcoma)
See NCCN Non-Hodgkin's

Lymphoma Guidelines

aBiopsy:

- Preferred route is transnasal.

- Needle biopsy may be acceptable.

- Avoid canine fossa puncture or Caldwell-Luc approach.

${ }^{b}$ For sinonasal undifferentiated carcinomas (SNUC), esthesioneuroblastoma, and small cell neuroendocrine histologies, systemic therapy should be a part of the overall treatment.

Note: All recommendations are category $2 \mathrm{~A}$ unless otherwise indicated.

Clinical Trials: NCCN believes that the best management of any cancer patient is in a clinical trial. Participation in clinical trials is especially encouraged. 


\section{NCCN ${ }^{\oplus}$ Practice Guidelines in Oncology - v.2.2008 \\ Head and Neck Cancers \\ Maxillary Sinus Tumors}

\section{STAGING}

PRIMARY TREATMENT

ADJUVANT TREATMENT

\section{FOLLOW-UP}

T1-2, N0 All histologies except adenoid cystic

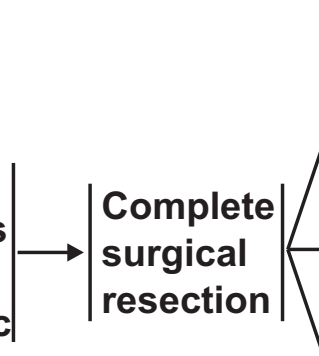
Margin negative negative Perineural invasion $\rightarrow$ Consider chemo/RT

Margin $\longrightarrow$ Surgical reresection positive $\longrightarrow$ if possible

\section{Consider $\mathrm{RT}^{\mathrm{C}}$}

or

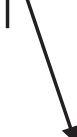

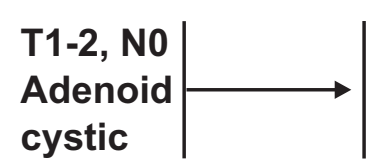

Complete surgical resection (category 2B)

$$
\text { if possible }
$$

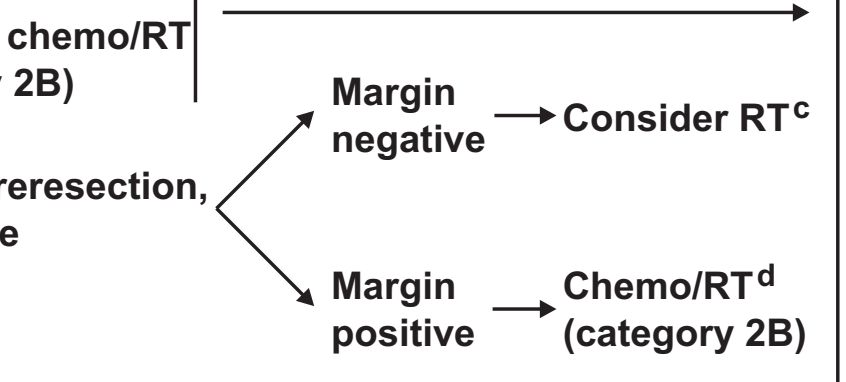

$R^{c}$

\author{
- Physical exam: \\ - Year 1, \\ every 1-3 mo \\ - Year 2, \\ every 2-4 mo \\ - Years 3-5, \\ every 4-6 mo \\ $>5$ years, \\ every 6-12 mo \\ - Chest imaging as clinically \\ indicated \\ - TSH every 6-12 mo, if neck \\ irradiated \\ - CT/MRI- baseline (category 2B)
}




\section{$\mathrm{NCCN}^{\oplus}$ Practice Guidelines in Oncology - v.2.2008 \\ Head and Neck Cancers \\ Maxillary Sinus Tumors}

\section{STAGING}

PRIMARY TREATMENT

ADJUVANT TREATMENT

FOLLOW-UP

'See Principles of Radiation Therapy (MAXI-A).

d See Principles of Systemic Therapy (CHEM-A).

eAdverse features include positive margins or extracapsular nodal spread.

Note: All recommendations are category $2 \mathrm{~A}$ unless otherwise indicated.

Clinical Trials: NCCN believes that the best management of any cancer patient is in a clinical trial. Participation in clinical trials is especially encouraged. 


\section{$\mathrm{NCCN}^{\oplus}$ Practice Guidelines

\section{PRINCIPLES OF RADIATION THERAPY}

\section{Definitive RT}

- Primary and gross adenopathy:

$\geq 66$ Gy (2.0 Gy/day)

- Neck

Uninvolved nodal stations:

$\geq 50$ Gy (2.0 Gy/day)

Postoperative RT

- Primary: $\geq 60$ Gy (2.0 Gy/day)

- Neck

- Involved nodal stations:

$\geq 60$ Gy (2.0 Gy/day)

Uninvolved nodal stations:

$\geq 50$ Gy (2.0 Gy/day) 


\section{$\mathrm{NCCN}{ }^{\oplus}$ Practice Guidelines in Oncology - v.2.2008

WORKUP

See Workup and Primary

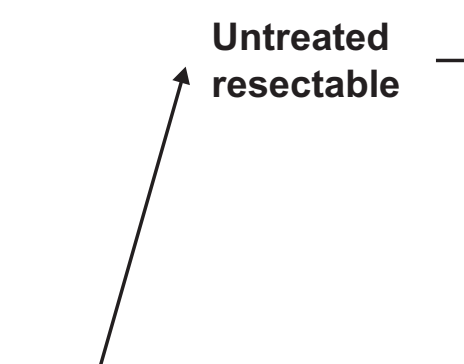

Salivary gland mass

- Parotid

- Submaxillary

- Minor salivary

gland $^{\mathrm{a}}$

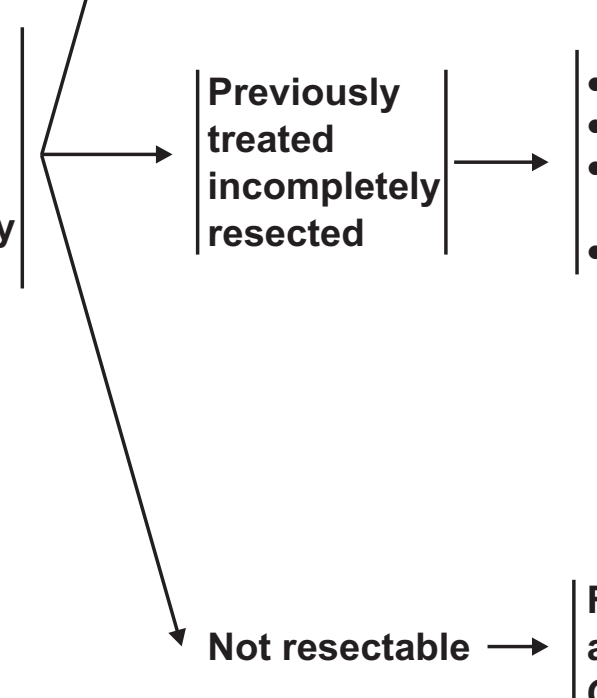

$\left|\begin{array}{l}\text { Fine-needle } \\ \text { aspiration or } \\ \text { Open biopsy }\end{array}\right|$
TREATMENT

a Site and stage determine therapeutic approaches.

b See Principles of Radiation Therapy (SALI-A).

Note: All recommendations are category $2 \mathrm{~A}$ unless otherwise indicated.

Clinical Trials: NCCN believes that the best management of any cancer patient is in a clinical trial. Participation in clinical trials is especially encouraged. 


\section{NCCN ${ }^{\oplus}$ Practice Guidelines in Oncology - v.2.2008

WORKUP

Untreated resectable,
clinically benign, ${ }^{\mathrm{c}}$
$<4 \mathrm{~cm}(\mathrm{~T} 1, \mathrm{~T} 2)$

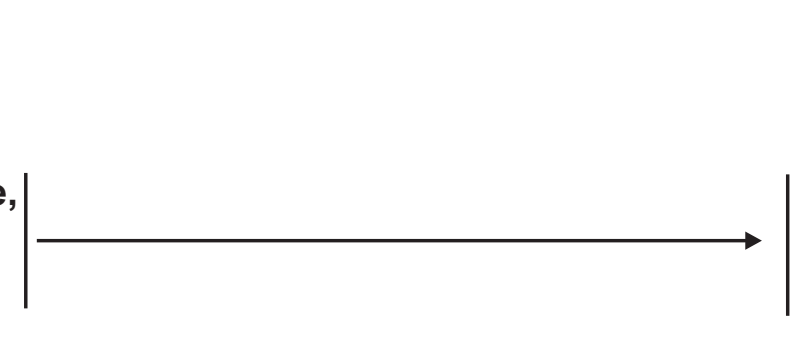

\section{PRIMARY TREATMENT}

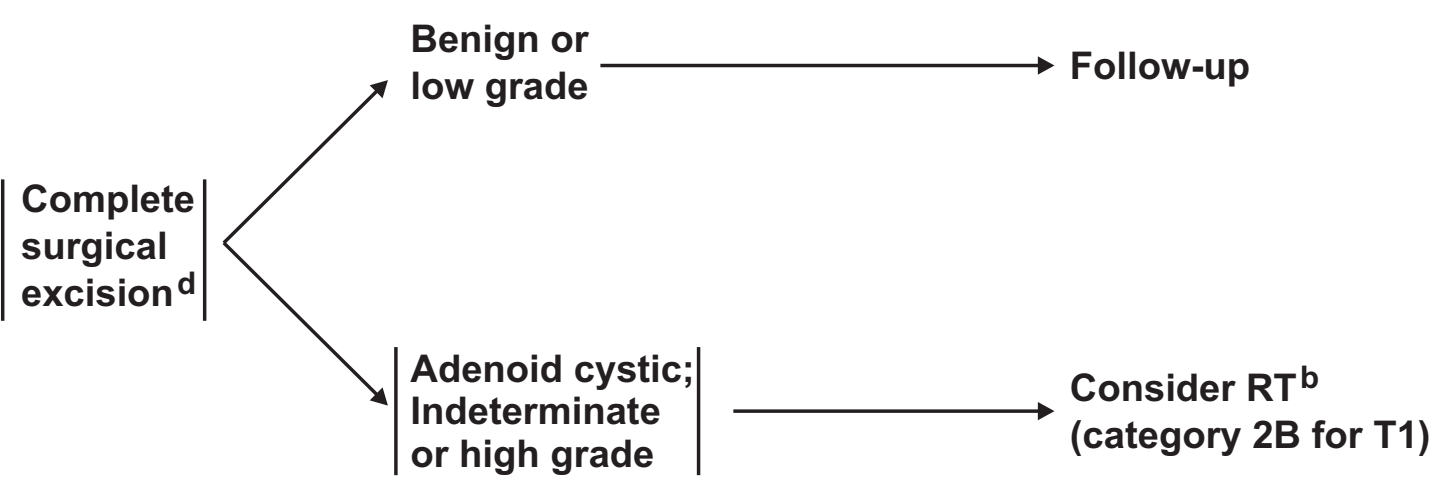

\section{Untreated}

resectable, clinically

suspicious for

cancer,

$>4 \mathrm{~cm}$ or deep lobe

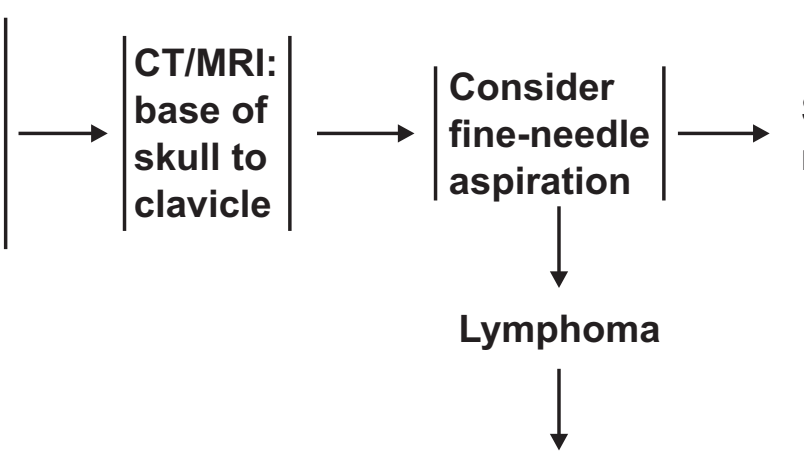

See NCCN Non-Hodgkin's

Lymphoma Guidelines

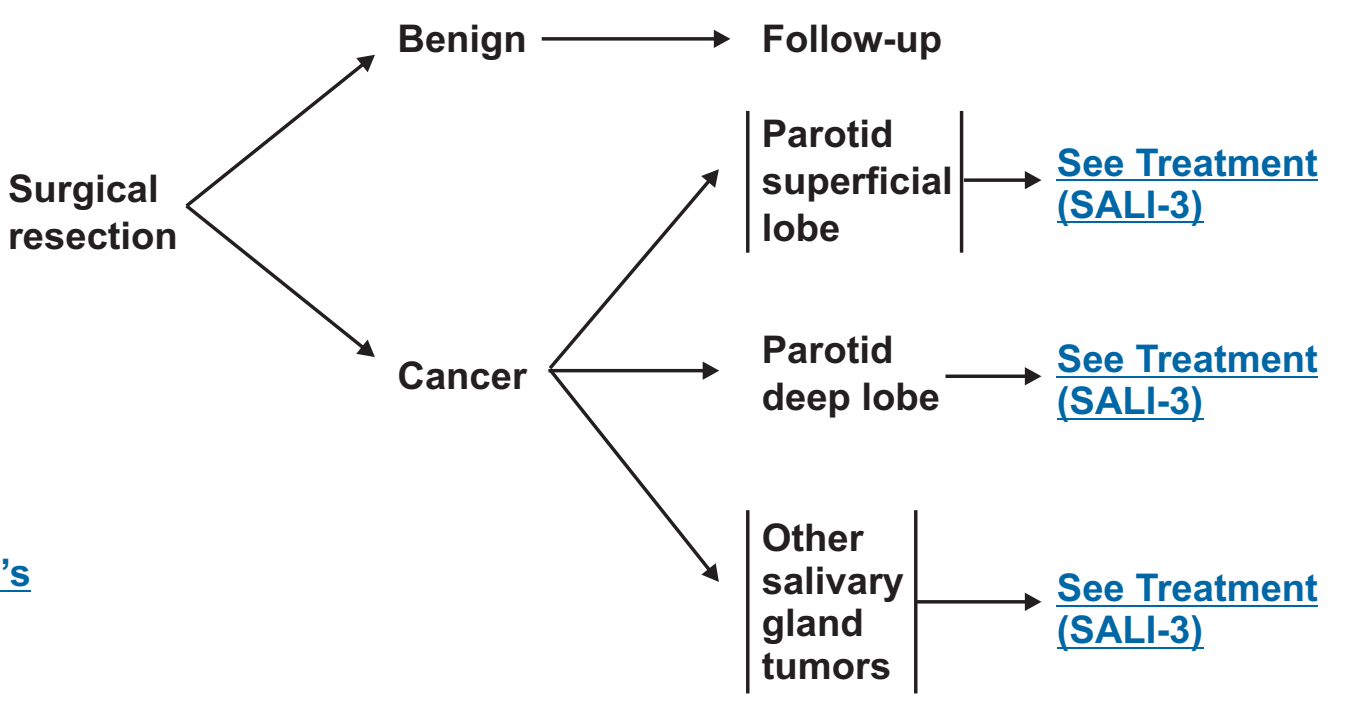

${ }^{b}$ See Principles of Radiation Therapy (SALI-A).

${ }^{\mathrm{C}}$ Characteristics of benign tumor include mobile superficial lobe, slow growth, painless, VII intact, and no neck nodes.

dSurgical excision of clinically benign tumor: no enucleation of lateral lobe, intraoperative communication with pathologist if indicated.

Note: All recommendations are category $2 \mathrm{~A}$ unless otherwise indicated.

Clinical Trials: NCCN believes that the best management of any cancer patient is in a clinical trial. Participation in clinical trials is especially encouraged. 


\section{NCCN ${ }^{\oplus}$ Practice Guidelines

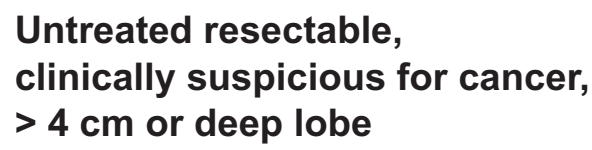

Untreated resectable, clinically suspicious for cancer, $>4 \mathrm{~cm}$ or deep lobe

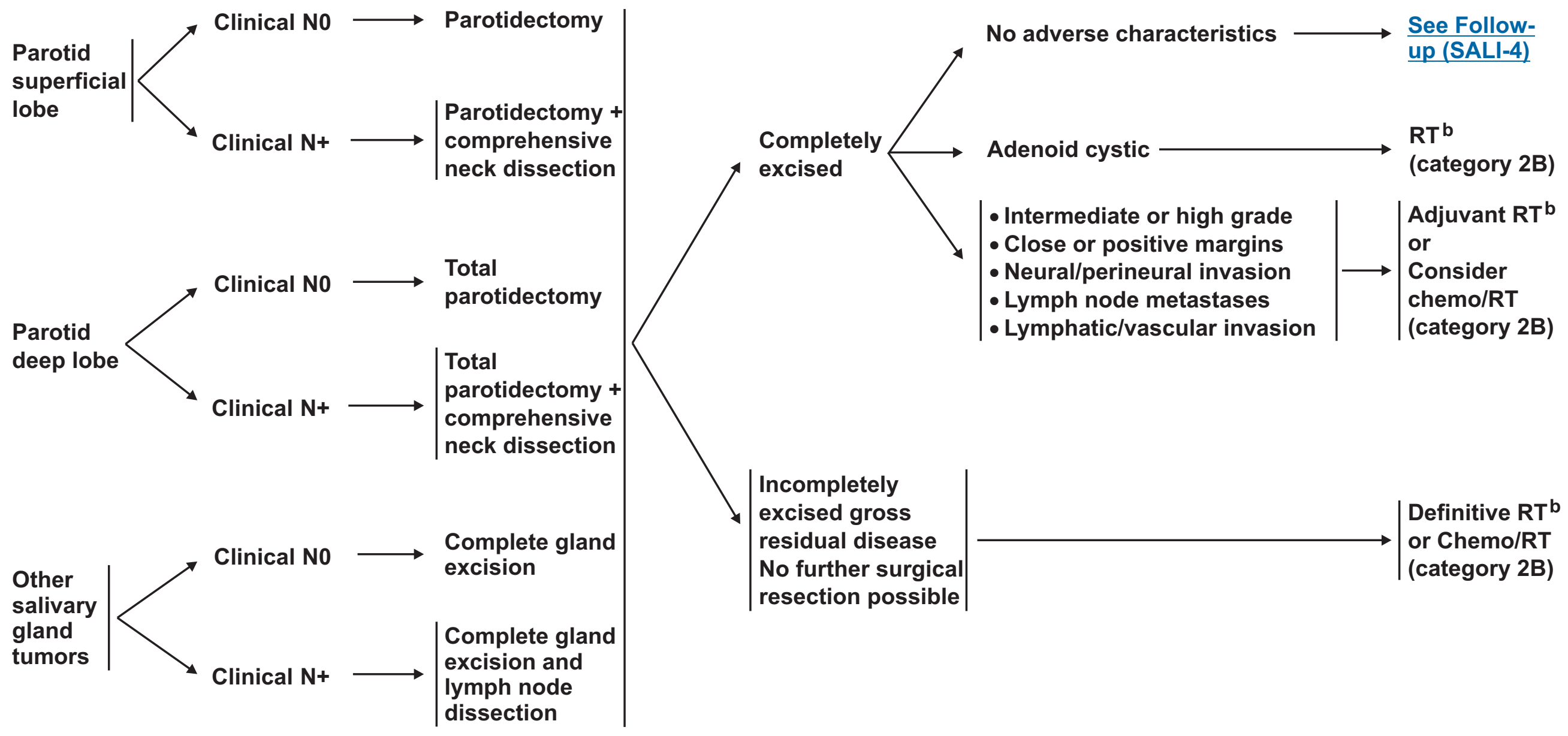

\section{TREATMENT}

Recurrence (see SALI-4)

Note: All recommendations are category $2 \mathrm{~A}$ unless otherwise indicated.

Clinical Trials: NCCN believes that the best management of any cancer patient is in a clinical trial. Participation in clinical trials is especially encouraged. 


\section{FOLLOW-UP}

- Physical exam:

- Year 1, every 1-3 mo

- Year 2, every 2-4 mo

- Years 3-5, every 4-6 mo

$>5$ yr, every 6-12 mo

- Chest imaging as

clinically indicated

- TSH every 6-12 mo, if neck irradiated

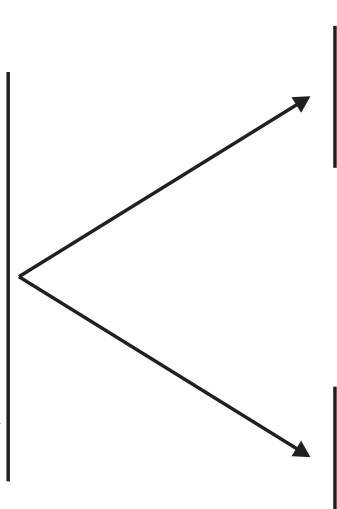

Locoregional or distant disease; Resectable

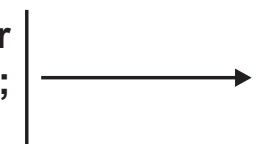

Surgery or selected metastasectomy (category 3)

$R T^{b}$

b See Principles of Radiation Therapy (SALI-A).

Note: All recommendations are category $2 \mathrm{~A}$ unless otherwise indicated.

Clinical Trials: NCCN believes that the best management of any cancer patient is in a clinical trial. Participation in clinical trials is especially encouraged. 


\section{$\mathrm{NCCN}^{\oplus}$ Practice Guidelines

PRINCIPLES OF RADIATION THERAPY

Definitive RT

Unresectable disease or gross residual disease

- Photon/electron therapy or neutron therapy

- Dose

- Primary and gross adenopathy:

$\geq 70$ Gy $(1.8-2.0 \mathrm{~Gy} /$ day $){ }^{1}$ or 19.2 nGy (1.2 nGy/day)

- Uninvolved nodal stations:

45-54 Gy (1.8-2.0 Gy/day) ${ }^{1}$ or 13.2 nGy (1.2 nGy/day)

Postoperative RT

- Photon/electron therapy or neutron therapy

- Dose

- Primary: $\geq 60$ Gy $(1.8-2.0 \text { Gy/day })^{1}$

or $18 \mathrm{nGy}$ (1.2 nGy/day)

- Neck: 45-54 Gy (1.8-2.0 Gy/day) ${ }^{1}$

or $13.2 \mathrm{nGy}(1.2 \mathrm{nGy} /$ day)

${ }^{1}$ Range based on grade/natural history of disease (eg, 1.8 Gy fraction may be used for slower growing tumors).

Back to Workup and Primary Treatment

Note: All recommendations are category $2 \mathrm{~A}$ unless otherwise indicated.

Clinical Trials: NCCN believes that the best management of any cancer patient is in a clinical trial. Participation in clinical trials is especially encouraged. 


\section{$\mathrm{NCCN}^{\circ}$

\section{WORKUP}

- H\&P

- Biopsy

- Chest imaging

- As indicated for

primary evaluation

- Panorex

- CT/MRI

- Preanesthesia studies

- Dental evaluation

Multidisciplinary

consultation as

indicated

\section{CLINICAL STAGING}

T1-2, NO

See Treatment of Primary and Neck (LIP-2)

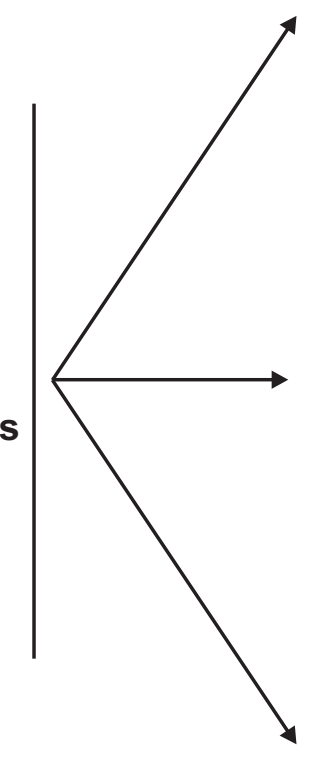

Resectable

T3, T4a, NO

Any T, N1-3

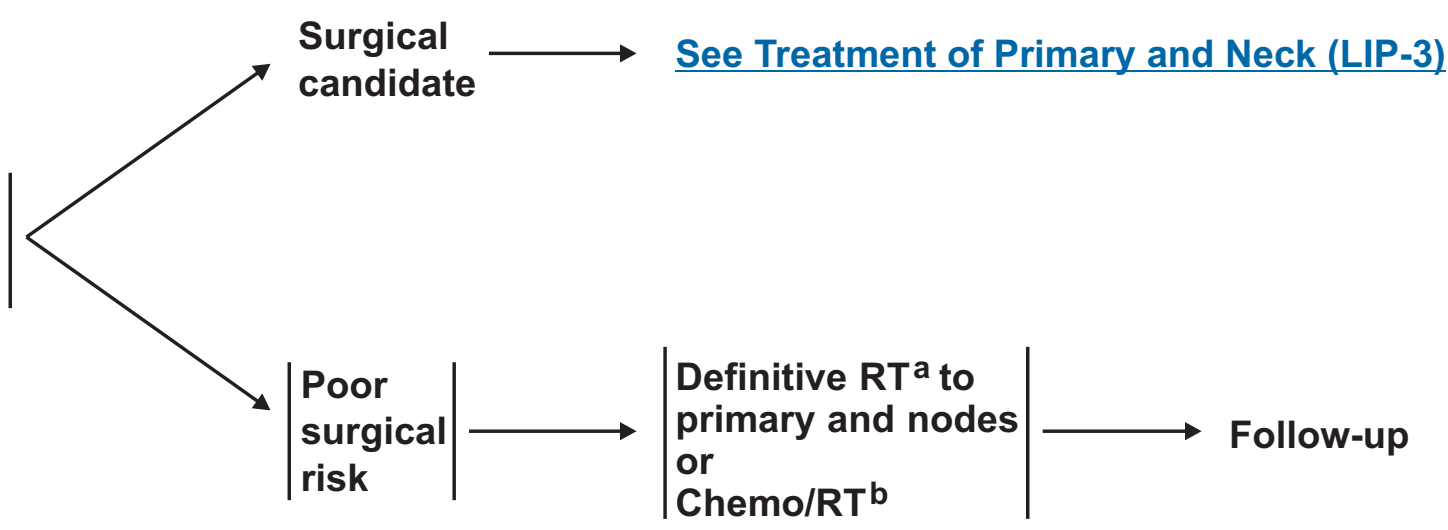

Unresectable

a See Principles of Radiation Therapy (LIP-A).

b See Principles of Systemic Therapy (CHEM-A).

Note: All recommendations are category $2 \mathrm{~A}$ unless otherwise indicated.

Clinical Trials: NCCN believes that the best management of any cancer patient is in a clinical trial. Participation in clinical trials is especially encouraged. 


\section{NCCN ${ }^{\oplus}$ Practice Guidelines in Oncology - v.2.2008

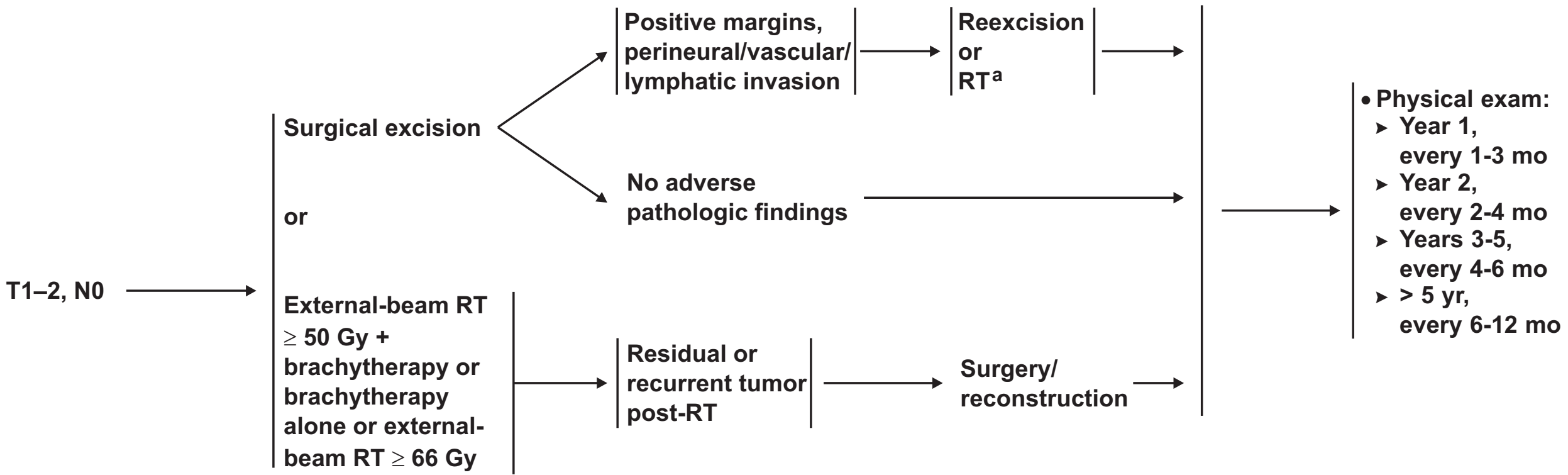

Note: All recommendations are category $2 \mathrm{~A}$ unless otherwise indicated.

Clinical Trials: NCCN believes that the best management of any cancer patient is in a clinical trial. Participation in clinical trials is especially encouraged. 
CLINICAL STAGING: RESECTABLE T3, T4a, N0; Any $\mathrm{T}, \mathrm{N} 1-3$

or

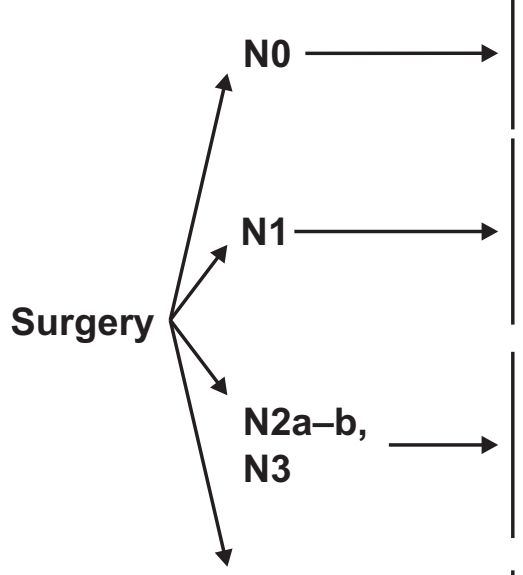

N2c (bilateral) $\rightarrow \mid \begin{aligned} & \text { Exc } \\ & \text { (rec }\end{aligned}$ TREATMENT OF PRIMARY AND NECK

ADJUVANT TREATMENT

Excision of primary \pm unilateral or bilateral selective neck dissection $\longrightarrow$ (reconstruction as indicated)

Excision of primary, ipsilateral selective or comprehensive neck dissection \pm contralateral selective neck dissection (reconstruction as indicated)

Excision of primary, ipsilateral comprehensive neck dissection \pm contralateral selective neck dissection (reconstruction as indicated) Excision of primary and bilateral (reconstruction as indicated)
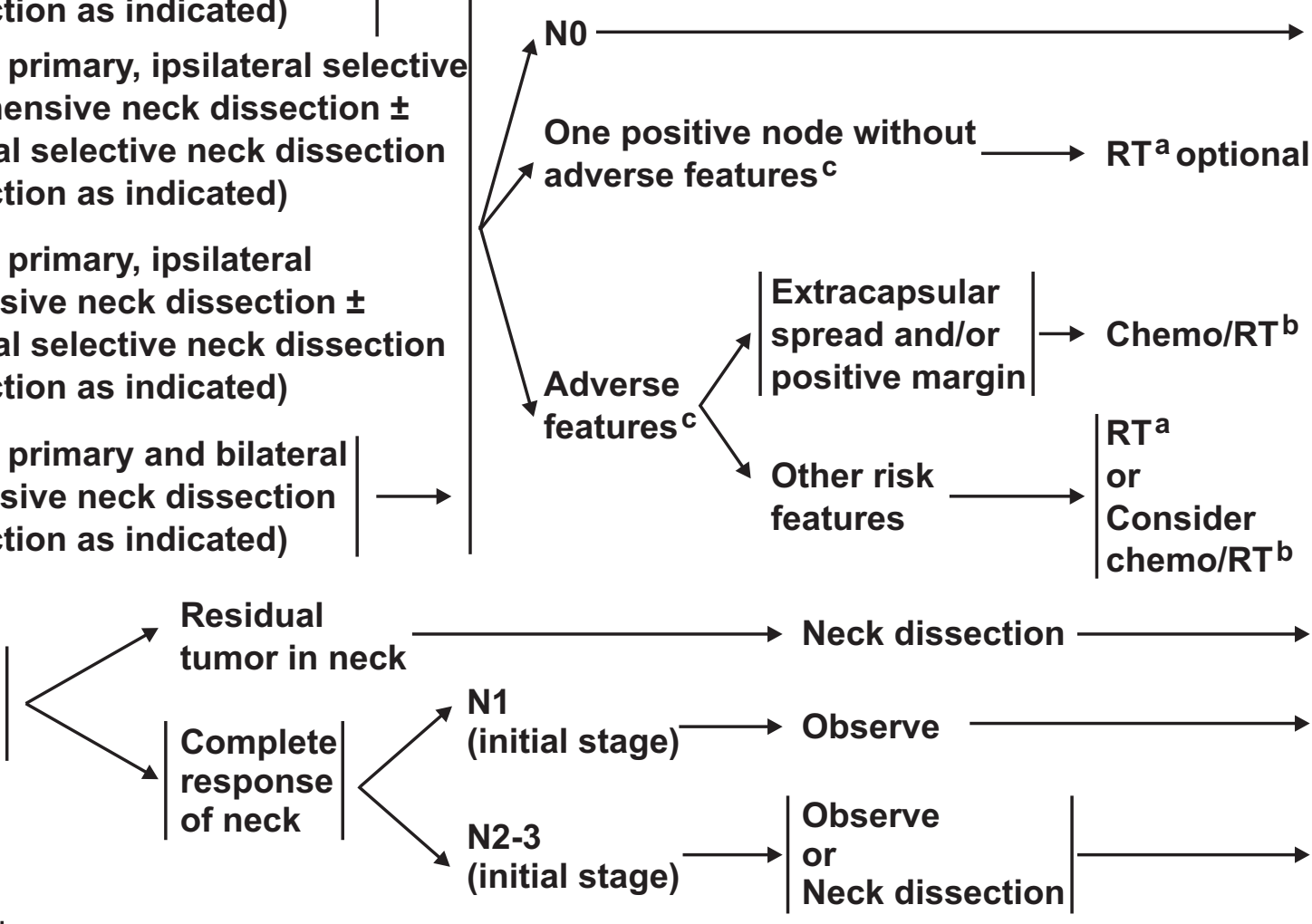

Neck dissection

Observe

Observe

or

Neck dissection
Salvage surgery + neck dissection as indicated

$\left|\begin{array}{l}\text { Primary site: } \\ <\text { complete } \\ \text { response }\end{array}\right|$

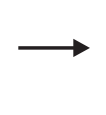

a See Principles of Radiation Therapy (LIP-A).

b See Principles of Systemic Therapy (CHEM-A).

${ }^{\mathrm{c}}$ Risk features: extracapsular nodal spread, positive margins, multiple positive nodes or perineural/lymphatic/vascular invasion.

Note: All recommendations are category $2 \mathrm{~A}$ unless otherwise indicated.

Clinical Trials: NCCN believes that the best management of any cancer patient is in a clinical trial. Participation in clinical trials is especially encouraged. 


\section{NCCN ${ }^{\oplus}$ Practice Guidelines \\ Head and Neck Cancers

PRINCIPLES OF RADIATION THERAPY

\begin{tabular}{|l|}
\hline \\
Definitive RT \\
- Primary and gross adenopathy: \\
$\geq 66$ Gy (2.0 Gy/day) \\
External-beam RT $\geq 50$ Gy + \\
brachytherapy or brachytherapy alone \\
- Neck \\
Uninvolved nodal stations: \\
$\geq 50$ Gy (2.0 Gy/day) \\
Postoperative RT \\
Primary: $\geq 60$ Gy (2.0 Gy/day) \\
$\bullet$ Neck \\
- Involved nodal stations: \\
$\geq 60$ Gy (2.0 Gy/day) \\
- Uninvolved nodal stations: \\
$\geq 50$ Gy (2.0 Gy/day) \\
\end{tabular}

Note: All recommendations are category $2 \mathrm{~A}$ unless otherwise indicated.

Clinical Trials: NCCN believes that the best management of any cancer patient is in a clinical trial. Participation in clinical trials is especially encouraged. 
Buccal mucosa, floor of mouth, anterior tongue, alveolar ridge, retromolar trigone, hard palate

\section{WORKUP}

- H\&P

- Biopsy

- Chest imaging

- CT/MRI as indicated

- Examination under

anesthesia, if indicated

- Preanesthesia studies

- Dental evaluation,

including panorex as indicated

Multidisciplinary

consultation as indicated

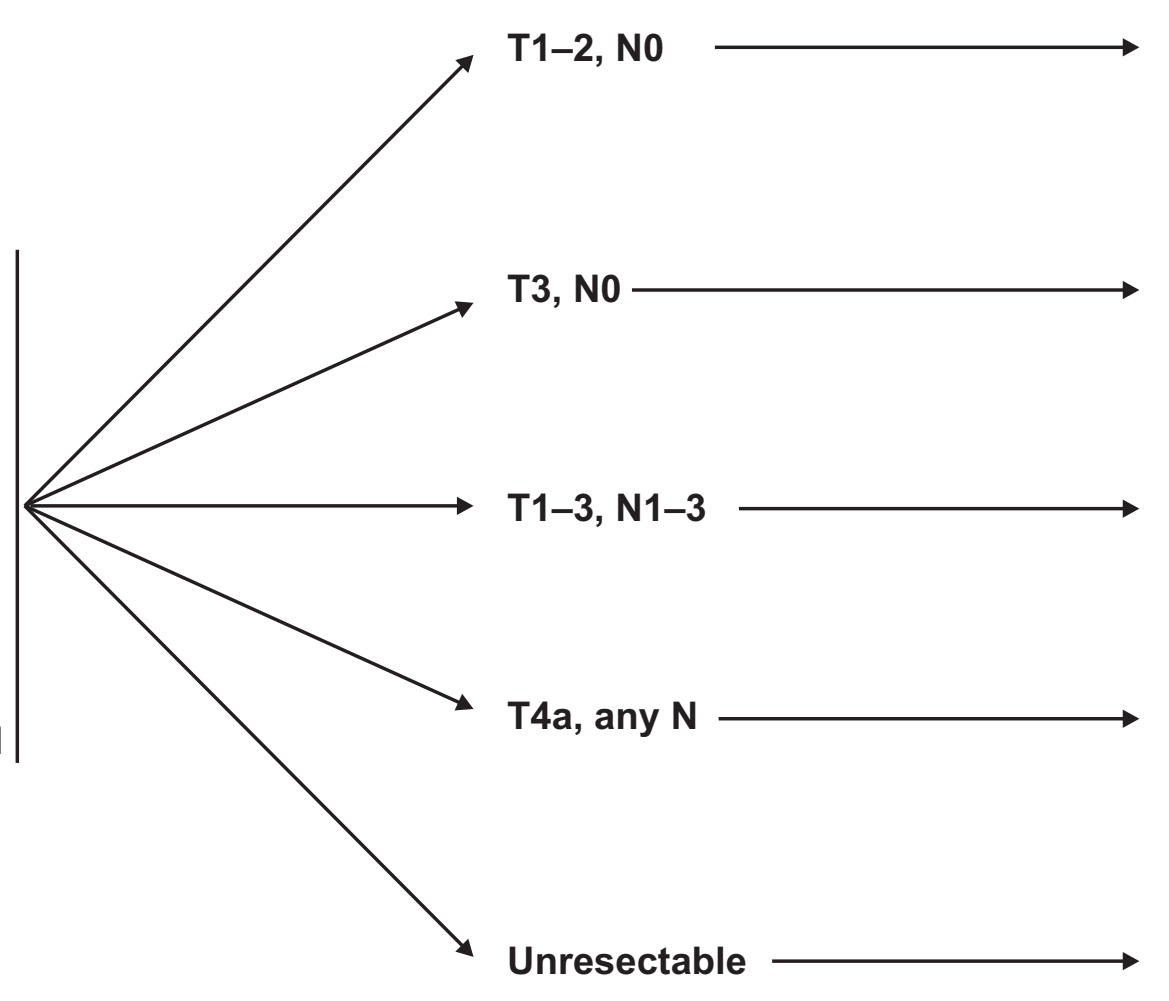

See Treatment of Head and Neck Cancer (ADV-1)

Note: All recommendations are category $2 \mathrm{~A}$ unless otherwise indicated.

Clinical Trials: NCCN believes that the best management of any cancer patient is in a clinical trial. Participation in clinical trials is especially encouraged. 
Buccal mucosa, floor of mouth, anterior tongue, alveolar ridge, retromolar trigone, hard palate
CLINICAL TREATMENT OF PRIMARY AND NECK
STAGING
ADJUVANT TREATMENT
Excision of primary (preferred) \pm unilateral or bilateral selective neck dissection

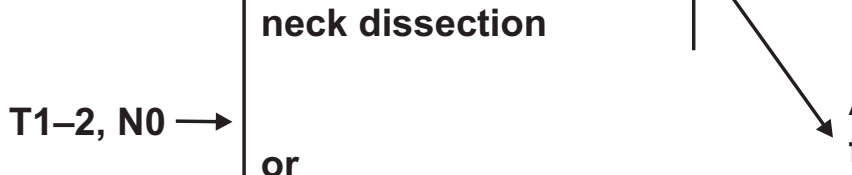 or

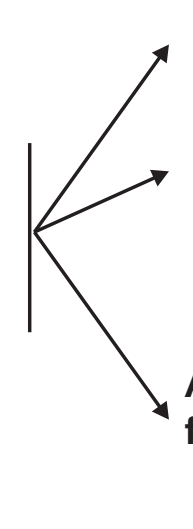
No adverse features ${ }^{a}$ One positive node without adverse features $^{a}$

FOLLOW-UP

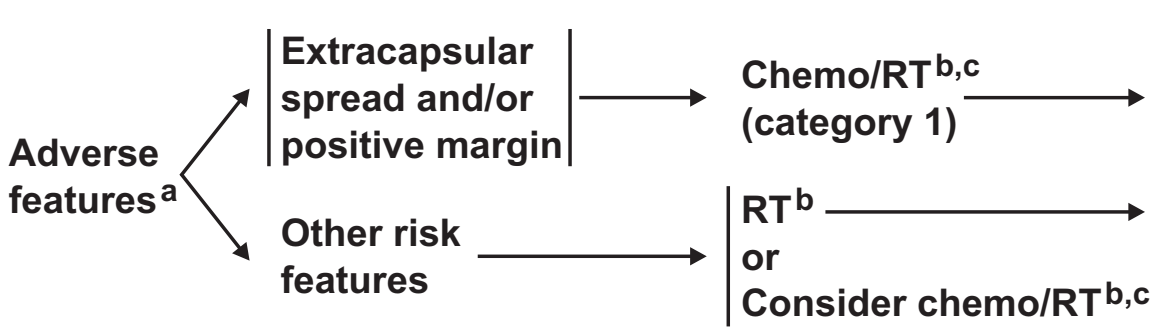

External-beam RT \pm brachytherapy $\geq 70$ Gy to primary $\geq \mathbf{5 0}$ Gy to neck at risk
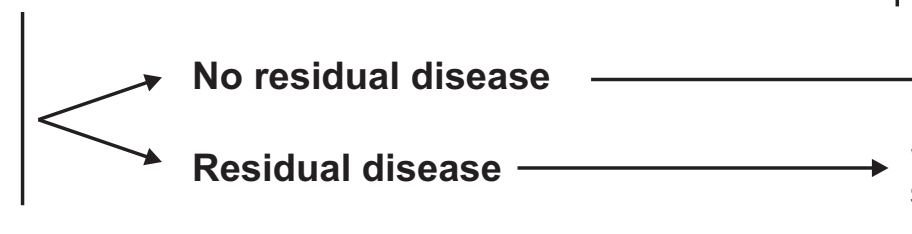
Salvage surgery

T3, NO $\longrightarrow$ Excision of primary and reconstruction as indicated and unilateral or bilateral selective neck dissection

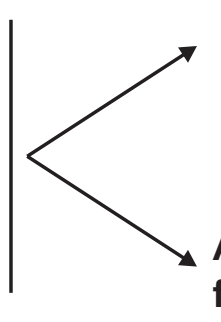

No adverse features ${ }^{a}$

$\mathrm{RT}^{\mathrm{b}}$ (optional)

Extracapsular spread and/or positive margin

Adverse features ${ }^{a}$

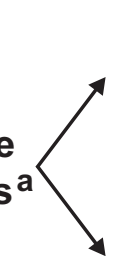

Other risk

features

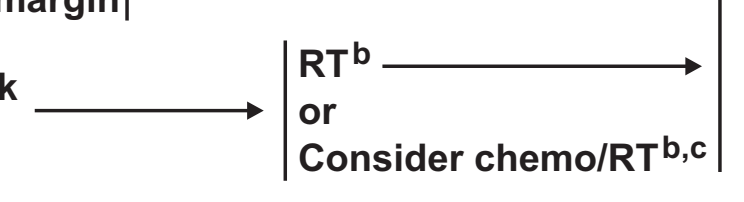

- Physical exam:

- Year 1, every 1-3 mo

- Year 2, every 2-4 mo

- Years 3-5, every 4-6 mo

> $>5 \mathrm{yr}$, every 6-12 mo

- Chest imaging as clinically indicated

- TSH every 6-12 mo, if neck irradiated

- Speech/hearing and swallowing evaluation and rehabilitation as indicated

- Smoking cessation counseling

- Dental follow-up recommended

a Risk features: extracapsular nodal spread, positive margins, pT3 or pT4 primary, N2 or N3 nodal disease,

nodal disease in levels IV or V, perineural invasion, vascular embolism.

b See Principles of Radiation Therapy (OR-A).

' See Principles of Systemic Therapy (CHEM-A).

Note: All recommendations are category $2 \mathrm{~A}$ unless otherwise indicated.

Clinical Trials: NCCN believes that the best management of any cancer patient is in a clinical trial. Participation in clinical trials is especially encouraged. 
Buccal mucosa, floor of mouth, anterior tongue, alveolar ridge, retromolar trigone, hard palate

CLINICAL STAGING
TREATMENT OF PRIMARY AND NECK
ADJUVANT

TREATMENT

\section{FOLLOW-UP}

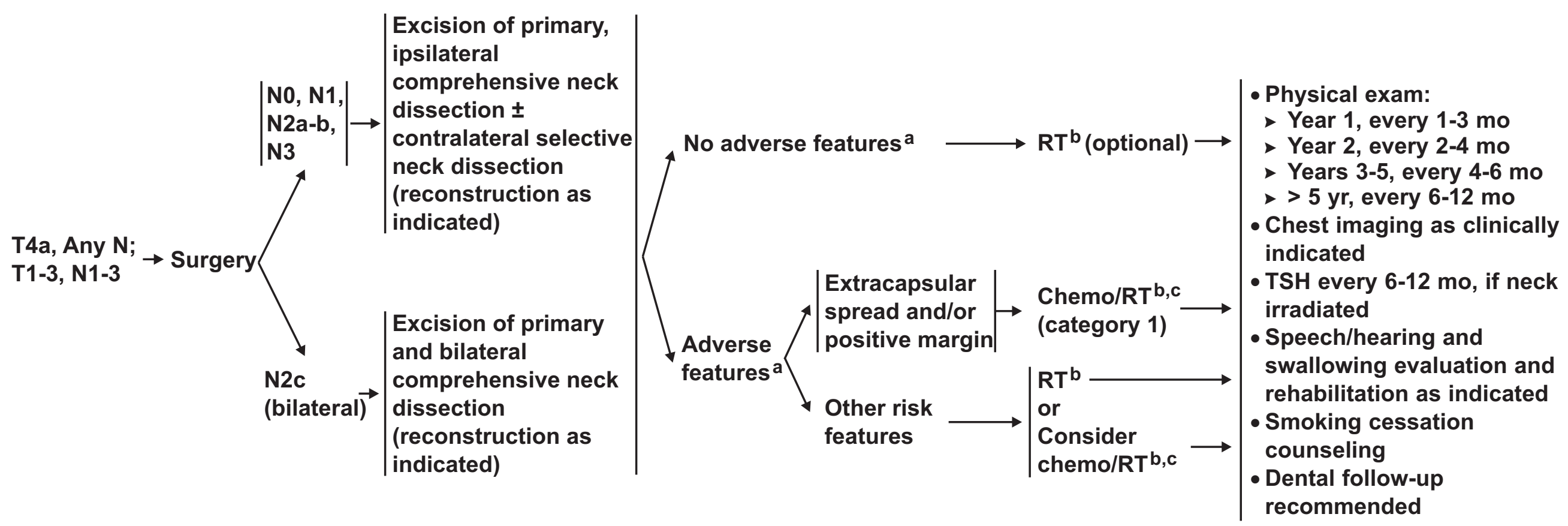

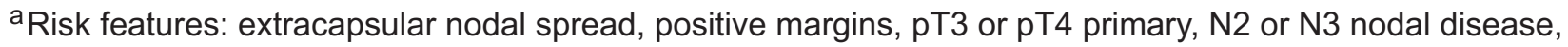

nodal disease in levels IV or V, perineural invasion, vascular embolism.

b See Principles of Radiation Therapy (OR-A).

'See Principles of Systemic Therapy (CHEM-A).

Note: All recommendations are category $2 \mathrm{~A}$ unless otherwise indicated.

Clinical Trials: NCCN believes that the best management of any cancer patient is in a clinical trial. Participation in clinical trials is especially encouraged. 


\begin{tabular}{|l|l|l|l} 
NCCN & $\begin{array}{l}\text { Practice Guidelines } \\
\text { in Oncology - v.2.2008 }\end{array}$ & $\begin{array}{l}\text { Head and Neck Cancers } \\
\text { Cancer of the Oral Cavity }\end{array}$ & $\begin{array}{l}\text { Head and N Neidelines Index } \\
\text { Staging, MS, Reers TOC }\end{array}$ \\
\hline
\end{tabular}

PRINCIPLES OF RADIATION THERAPY

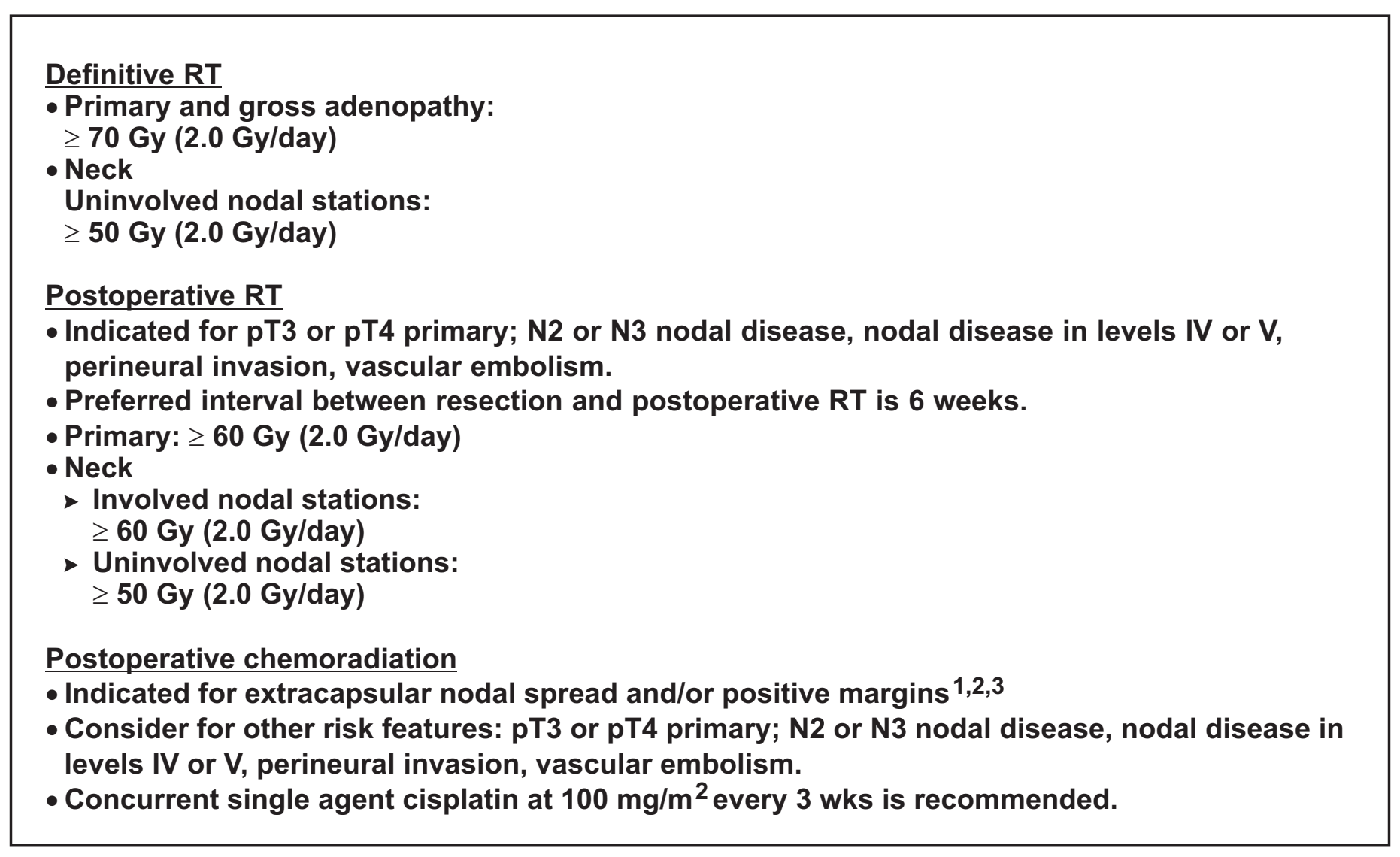

${ }^{1}$ Bernier J, Domenge C, Ozsahin M et al. Postoperative irradiation with or without concomitant chemotherapy for locally advanced head and neck cancer. N Engl J Med 2004;350:1945-1952.

${ }^{2}$ Cooper JS, Pajak TF, Forastiere AA et al. Postoperative concurrent radiotherapy and chemotherapy for high-risk squamous-cell carcinoma of the head and neck. N Engl J Med 2004;350(19):1937-1944.

${ }^{3}$ Bernier J, Cooper JS, Pajuk TF, et al. Defining risk levels in locally advanced head and neck cancers: A comparative analysis of concurrent postoperative radiation plus chemotherapy trials of the EORTC (\#22931) and RTOG (\#9501). Head Neck 2005;27:843-850.

Note: All recommendations are category $2 A$ unless otherwise indicated.

Clinical Trials: NCCN believes that the best management of any cancer patient is in a clinical trial. Participation in clinical trials is especially encouraged. 
Base of tongue/tonsil/posterior pharyngeal wall/soft palate WORKUP

CLINICAL STAGING

- H\&P

- Biopsy

- HPV testing suggested

- Chest imaging

- CT with contrast or MRI recommended for primary and neck

- Dental evaluation, including panorex as indicated

- Speech \& swallowing evaluation as indicated

- Examination under anesthesia with endoscopy

- Preanesthesia studies

Multidisciplinary consultation as indicated

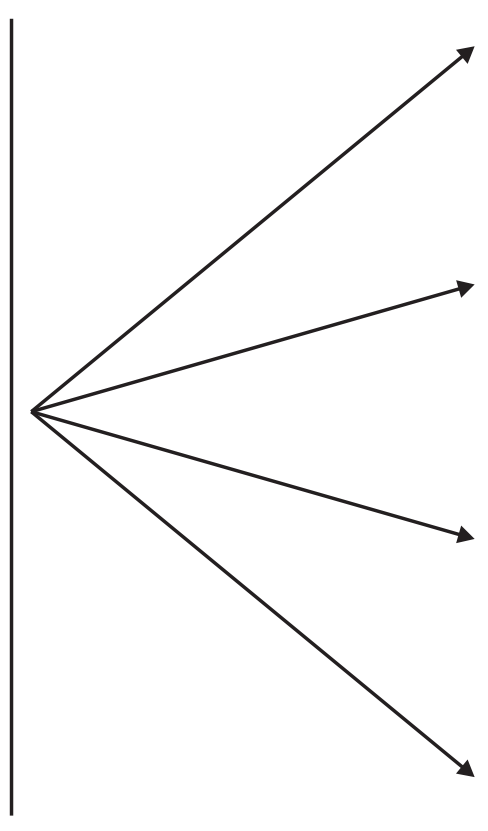

T1-2, N0-1

See Treatment of Primary and Neck (ORPH-2)

Any T, N2-3

T3-4a, N+

See Treatment of Primary and Neck (ORPH-4)

Unresectable

See Treatment of Head and Neck Cancer (ADV-1)

Note: All recommendations are category $2 \mathrm{~A}$ unless otherwise indicated.

Clinical Trials: NCCN believes that the best management of any cancer patient is in a clinical trial. Participation in clinical trials is especially encouraged. 
Base of tongue/tonsil/posterior pharyngeal wall/soft palate CLINICAL TREATMENT OF PRIMARY AND NECK STAGING

(n)

ADJUVANT $\quad$ FOLLOW-UP
TREATMENT

No-1

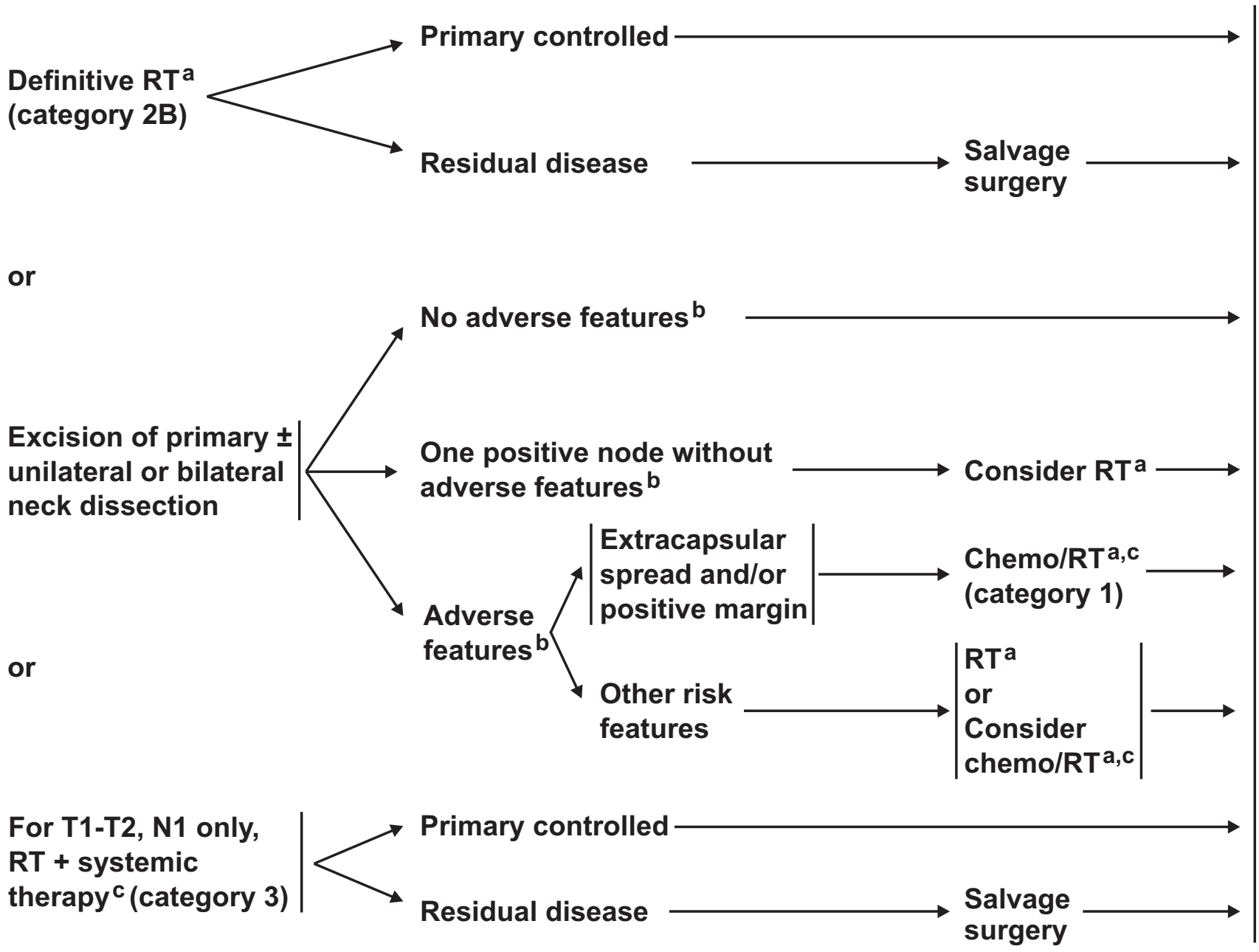

- Physical exam:

- Year 1,

every 1-3 mo

- Year 2,

every 2-4 mo

- Years 3-5,

every 4-6 mo

$>5 \mathrm{yr}$,

every 6-12 mo

- Chest imaging as clinically indicated

- TSH every 6-12 mo, if neck irradiated

- Speech, hearing and swallowing evaluation and rehabilitation as indicated

- Smoking cessation

counseling

- Dental evaluation as indicated

For T1-T2, N1 only, RT + systemic therapy ${ }^{\mathrm{C}}$ (category 3 ) surgery

a See Principles of Radiation Therapy (ORPH-A).

${ }^{b}$ Risk features: extracapsular nodal spread, positive margins, pT3 or pT4 primary, N2 or N3 nodal disease, nodal disease in levels IV or V, perineural invasion, vascular embolism.

'See Principles of Systemic Therapy (CHEM-A).

Note: All recommendations are category $2 \mathrm{~A}$ unless otherwise indicated.

Clinical Trials: NCCN believes that the best management of any cancer patient is in a clinical trial. Participation in clinical trials is especially encouraged. 
Base of tongue/tonsil/posterior pharyngeal wall/soft palate

CLINICAL TREATMENT OF PRIMARY AND NECK STAGING

ADJUVANT

FOLLOW-UP TREATMENT

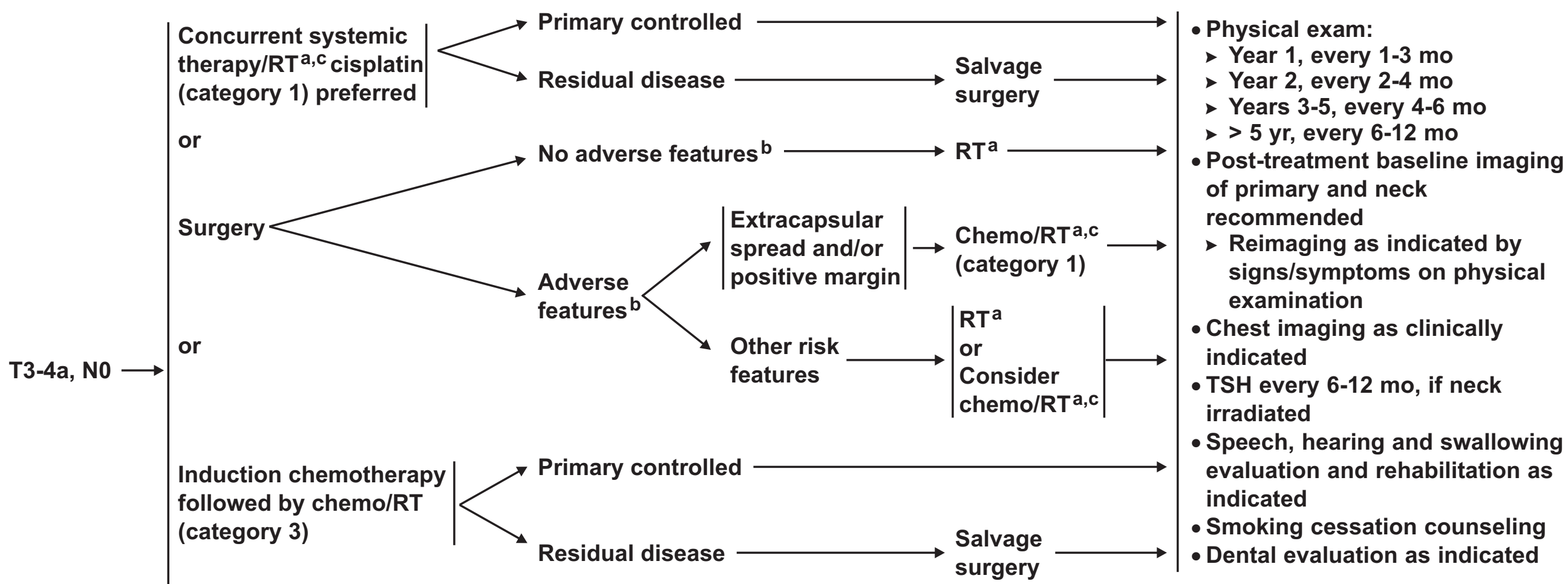

or

Recurrence (see ADV-2)

Multimodality clinical trials that

include function evaluation

a See Principles of Radiation Therapy (ORPH-A).

${ }^{b}$ Risk features: extracapsular nodal spread, positive margins, pT3 or pT4 primary, N2 or N3 nodal disease, nodal disease in levels IV or V, perineural invasion, vascular embolism.

'See Principles of Systemic Therapy (CHEM-A).

Note: All recommendations are category $2 \mathrm{~A}$ unless otherwise indicated.

Clinical Trials: NCCN believes that the best management of any cancer patient is in a clinical trial. Participation in clinical trials is especially encouraged. 
Base of tongue/tonsil/posterior pharyngeal wall/soft palate

CLINICAL TREATMENT OF PRIMARY AND NECK STAGING

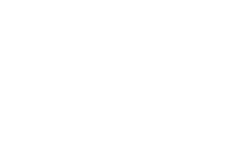

T3-4a, N+

or

Any T, N2-3$$
\text { TREATMENT OF PRIMARY AND NECK }
$$

\author{
(
}

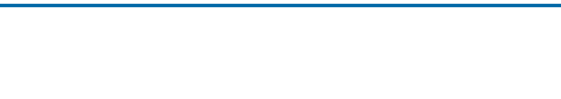

\section{FOLLOW-UP}

- Physical exam:

- Year 1, every 1-3 mo

- Year 2, every 2-4 mo

- Years 3-5, every 4-6 mo

> 5 yr, every 6-12 mo

- Post-treatment baseline imaging of primary and neck recommended

- Reimaging as indicated by signs/symptoms on physical examination

- Chest imaging as clinically indicated

- TSH every 6-12 mo, if neck irradiated

- Speech, hearing and swallowing evaluation and rehabilitation as indicated

- Smoking cessation counseling

- Dental evaluation as indicated 


\section{PRINCIPLES OF RADIATION THERAPY}

\section{Selected T1-2, NO}

- Conventional fractionation:

70 Gy (2.0 Gy/day)

Selected T1, N1; T2, N0-1

- Altered fractionation:

- Concomitant boost accelerated RT:

72 Gy/6 weeks (1.8 Gy/fraction, large field; 1.5

Gy boost as second daily fraction during last

12 treatment days)

- Hyperfractionation:

81.6 Gy/7 weeks (1.2 Gy/fraction BID)

Postoperative RT

- Indicated for pT3 or pT4 primary; N2 or N3 nodal disease, nodal disease in levels IV or V,

perineural invasion, vascular embolism.

- Preferred interval between resection and

postoperative RT is 6 weeks.

- Primary: $\geq 60$ Gy (2.0 Gy/day)

- Neck

- Involved nodal stations:

$\geq 60$ Gy (2.0 Gy/day)

- Uninvolved nodal stations:

$$
\geq 50 \text { Gy (2.0 Gy/day) }
$$

${ }_{1}^{1}$ Based on published data, concurrent chemoradiation typically uses conventional fractionation at $2.0 \mathrm{~g}$ per fraction to $\geq 70 \mathrm{~Gy}$ in 7 wks with single agent cisplatin given every 3 wks at $100 \mathrm{mg} / \mathrm{m}^{2} \times 3$ doses. Use of other fraction sizes (eg, $1.8 \mathrm{~Gy}$, conventional), multiagent chemotherapy, or altered fractionation with chemotherapy has been evaluated with no consensus on the optimal approach. In general, the use of concurrent chemoradiation carries a high toxicity burden--altered fractionation or multiagent chemotherapy will likely further increase toxicity burden. For any chemoradiation approach, close attention should be paid to published reports for the specific chemotherapy agent, dose, and schedule of administration. Chemoradiation should be performed by an experienced team and should include substantial supportive care.

2Bernier J, Domenge C, Ozsahin M, et al. Postoperative irradiation with or without concomitant chemotherapy for locally advanced head and neck cancer. N Engl J Med 2004;350:1945-1952.

${ }^{3}$ Cooper JS, Pajak TF, Forastiere AA, et al. Postoperative concurrent radiotherapy and chemotherapy for high-risk squamous-cell carcinoma of the head and neck. N Engl J Med 2004;350:1937-1944.

${ }^{4}$ Bernier J, Cooper JS, Pajuk TF, et al. Defining risk levels in locally advanced head and neck cancers: A comparative analysis of concurrent postoperative radiation plus chemotherapy trials of the EORTC (\#22931) and RTOG (\#9501). Head Neck 2005;27:843-850.

Note: All recommendations are category $2 \mathrm{~A}$ unless otherwise indicated.

Clinical Trials: NCCN believes that the best management of any cancer patient is in a clinical trial. Participation in clinical trials is especially encouraged. 


\section{NCCN ${ }^{\oplus}$ Practice Guidelines

WORKUP

- H\&P

- Biopsy

- Chest imaging

- CT with contrast or MRI of primary and neck recommended

- Examination under anesthesia with endoscopy

- Preanesthesia studies

- Speech \& swallowing

evaluation as indicated

- Dental evaluation

Multidisciplinary consultation as indicated

\section{CLINICAL STAGING}

Early T stage not requiring total laryngectomy

- Most T1, N0, small T2, NO

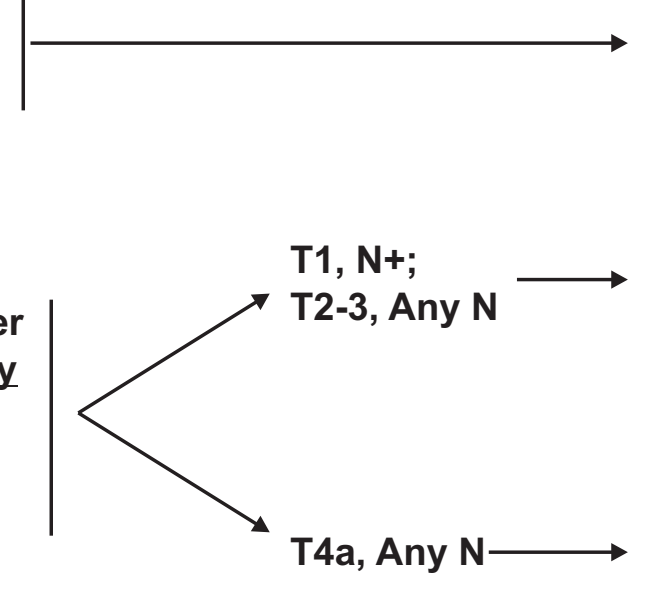

See Treatment of Primary and Neck (HYPO-2)

See Treatment of Primary and Neck (HYPO-5)

See Treatment of Head and Neck Cancer (ADV-1)

Note: All recommendations are category $2 \mathrm{~A}$ unless otherwise indicated.

Clinical Trials: NCCN believes that the best management of any cancer patient is in a clinical trial. Participation in clinical trials is especially encouraged. 


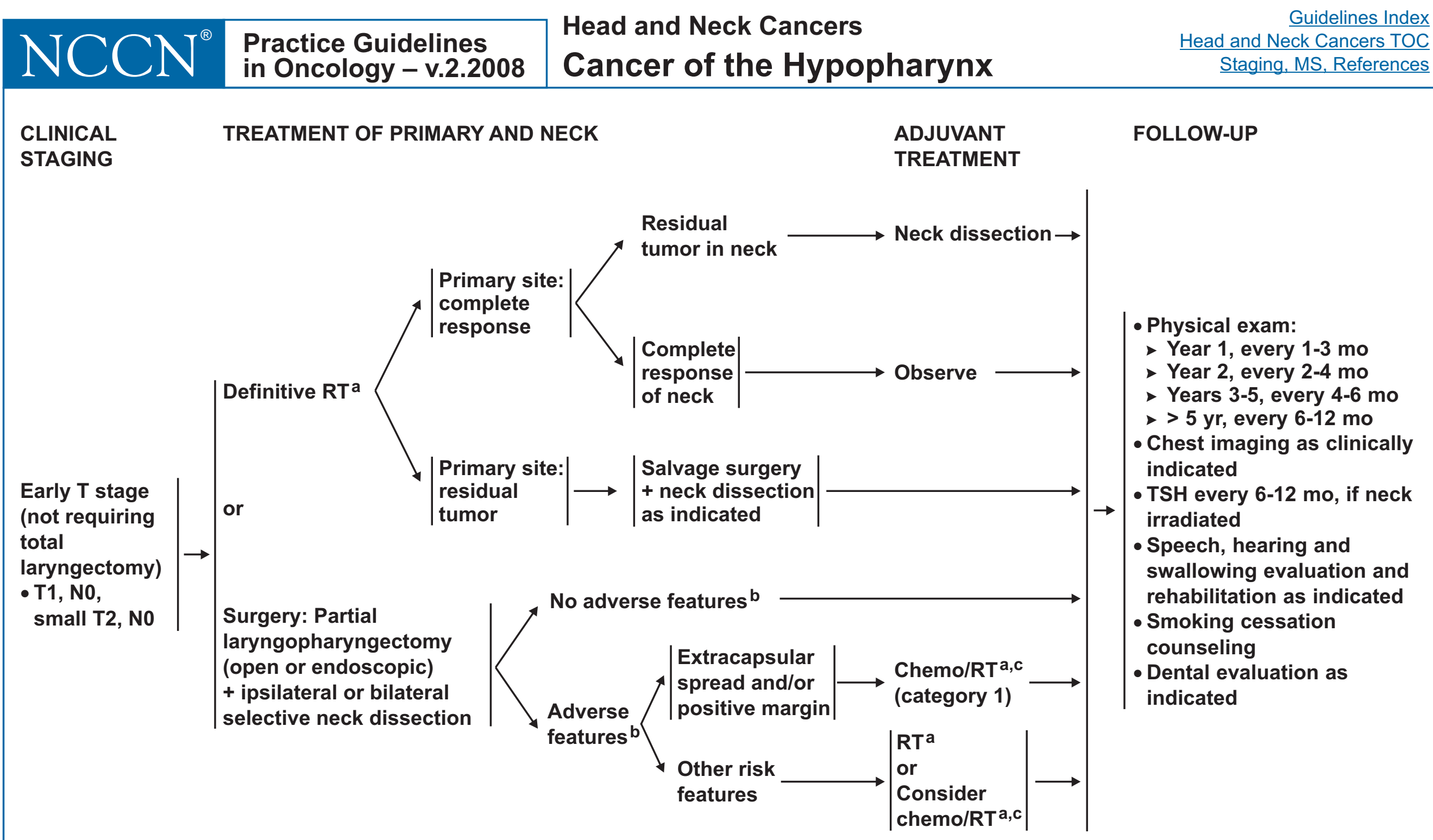

a See Principles of Radiation Therapy (HYPO-A).

${ }^{b}$ Risk features: extracapsular nodal spread, positive margins, pT3 or pT4 primary, N2 or N3 nodal disease, perineural invasion, vascular embolism.

'See Principles of Systemic Therapy (CHEM-A).

Note: All recommendations are category $2 \mathrm{~A}$ unless otherwise indicated.

Clinical Trials: NCCN believes that the best management of any cancer patient is in a clinical trial. Participation in clinical trials is especially encouraged. 


\section{CLINICAL STAGING}

$\mathrm{T} 1, \mathrm{~N}+$;

T2-3, any $N$ (if total laryngectomy required)

\section{TREATMENT OF PRIMARY AND NECK}

Induction chemotherapy ${ }^{c} \times 2$ cycles (category 1)

or Laryngopharyngectomy + selective (NO) or comprehensive $(\mathrm{N}+)$ neck dissection, including level VI

or

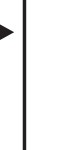

Concurrent systemic therapy/RTa,c cisplatin preferred (category 2B)

or

See Response After Induction

ADJUVANT

TREATMENT

\section{FOLLOW-UP}

Multimodality clinical

trials that include

function evaluation

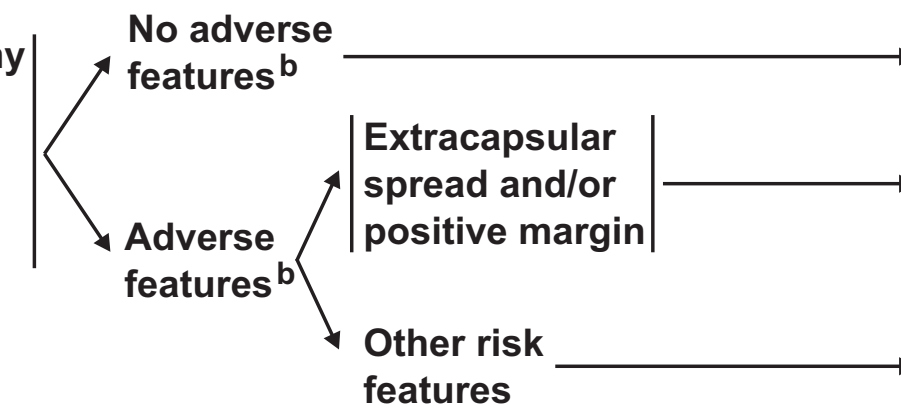
$\mathrm{RT}^{\mathrm{a}}$ Chemotherapy (HYPO-4) features

Chemo/RT ${ }^{a, c}$ (category 1)<smiles>[R17]1[c+]ccc1</smiles>
or Consider chemo/RT $^{a, c}$

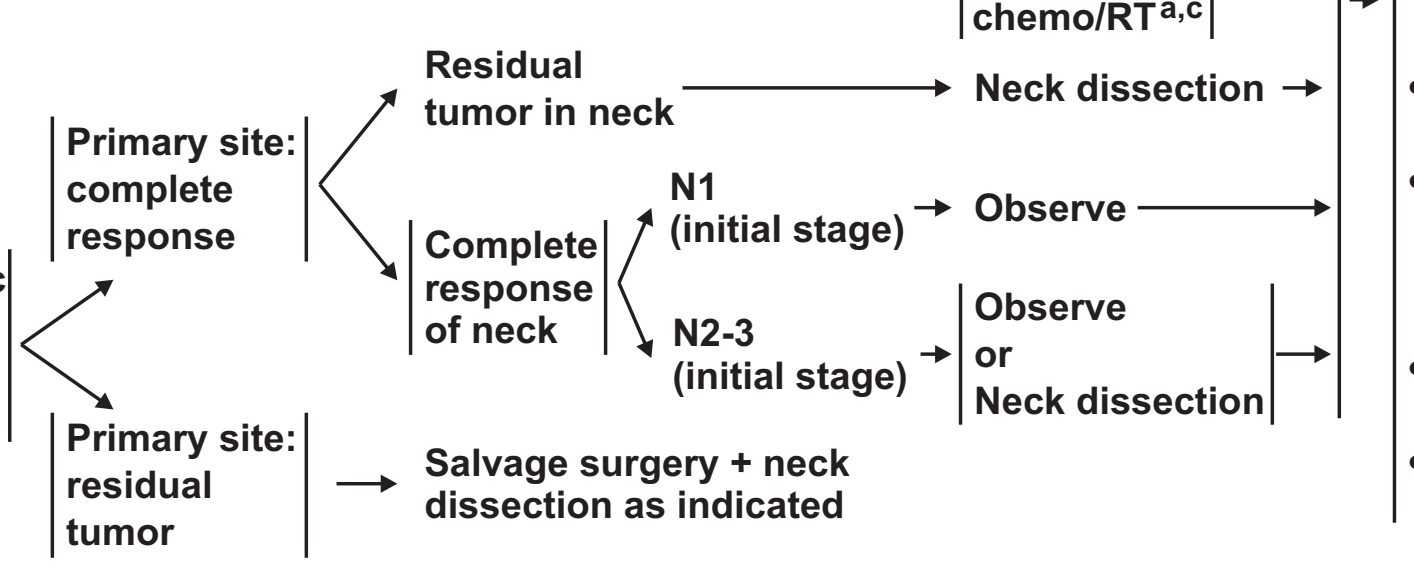

Residual

$\left|\begin{array}{l}\text { Complete } \\ \text { response } \\ \text { of neck }\end{array}\right|$

\section{N1}

N2-3

- Physical exam:

- Year 1, every 1-3 mo

- Year 2, every 2-4 mo

- Years 3-5, every 4-6 mo

> 5 yr, every 6-12 mo

- Post-treatment baseline imaging of primary and neck recommended

- Reimaging as indicated by signs/symptoms on physical examination

- Chest imaging as clinically indicated

- TSH every 6-12 mo, if neck irradiated

- Speech, hearing and swallowing evaluation and rehabilitation as indicated

- Smoking cessation counseling

- Dental evaluation as indicated

a See Principles of Radiation Therapy (HYPO-A).

${ }^{b}$ Risk features: extracapsular nodal spread, positive margins, pT3 or pT4 primary, N2 or N3 nodal disease, perineural invasion, vascular embolism.

'See Principles of Systemic Therapy (CHEM-A).

Note: All recommendations are category $2 \mathrm{~A}$ unless otherwise indicated.

Clinical Trials: NCCN believes that the best management of any cancer patient is in a clinical trial. Participation in clinical trials is especially encouraged. 


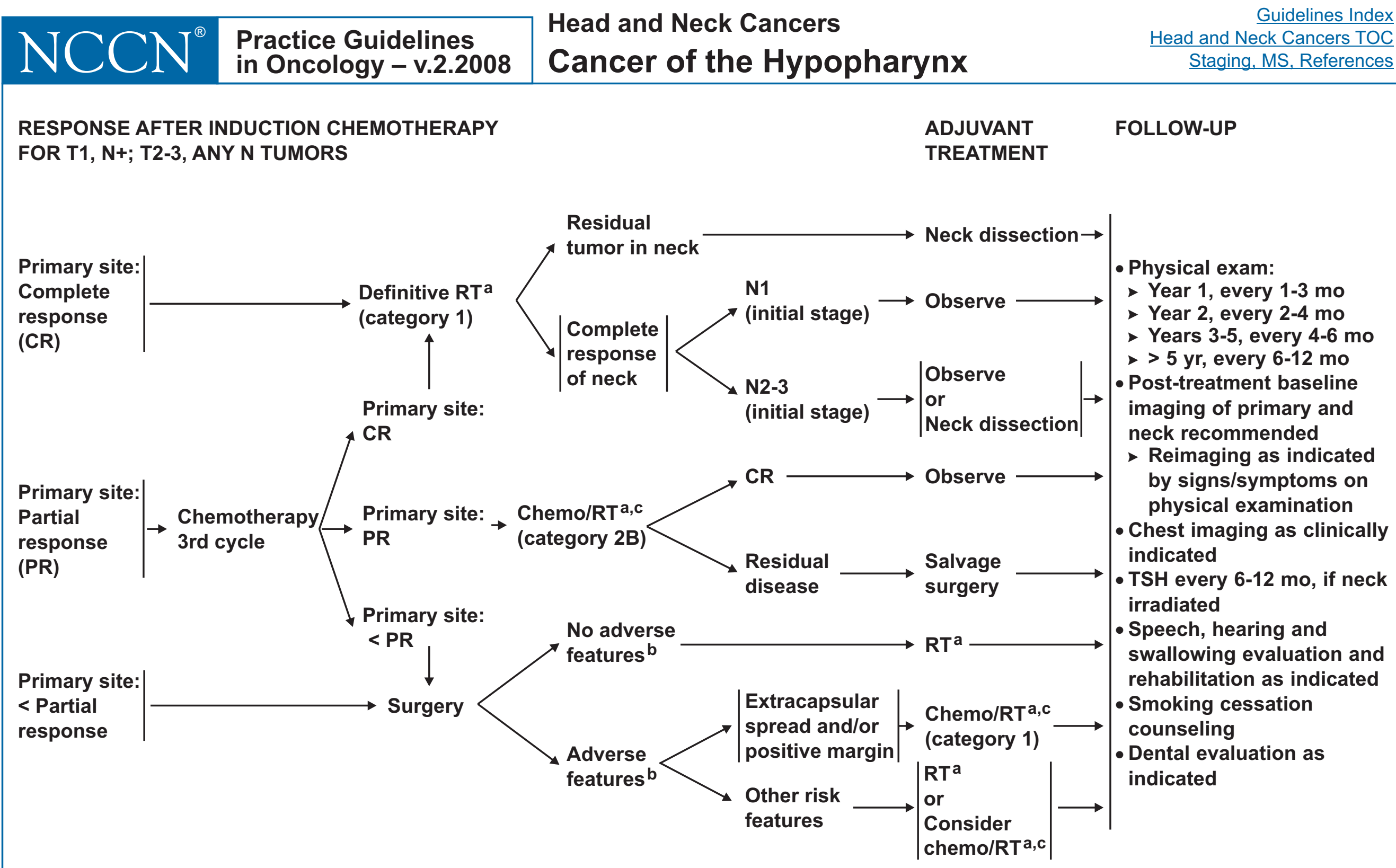

a See Principles of Radiation Therapy (HYPO-A).

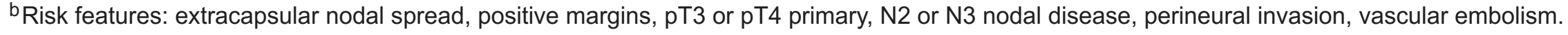

'See Principles of Systemic Therapy (CHEM-A).

Note: All recommendations are category $2 \mathrm{~A}$ unless otherwise indicated.

Clinical Trials: NCCN believes that the best management of any cancer patient is in a clinical trial. Participation in clinical trials is especially encouraged. 


\section{NCCN ${ }^{\oplus}$ Practice Guidelines

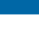

CLINICAL TREATMENT OF PRIMARY AND NECK

STAGING

ADJUVANT

TREATMENT

FOLLOW-UP

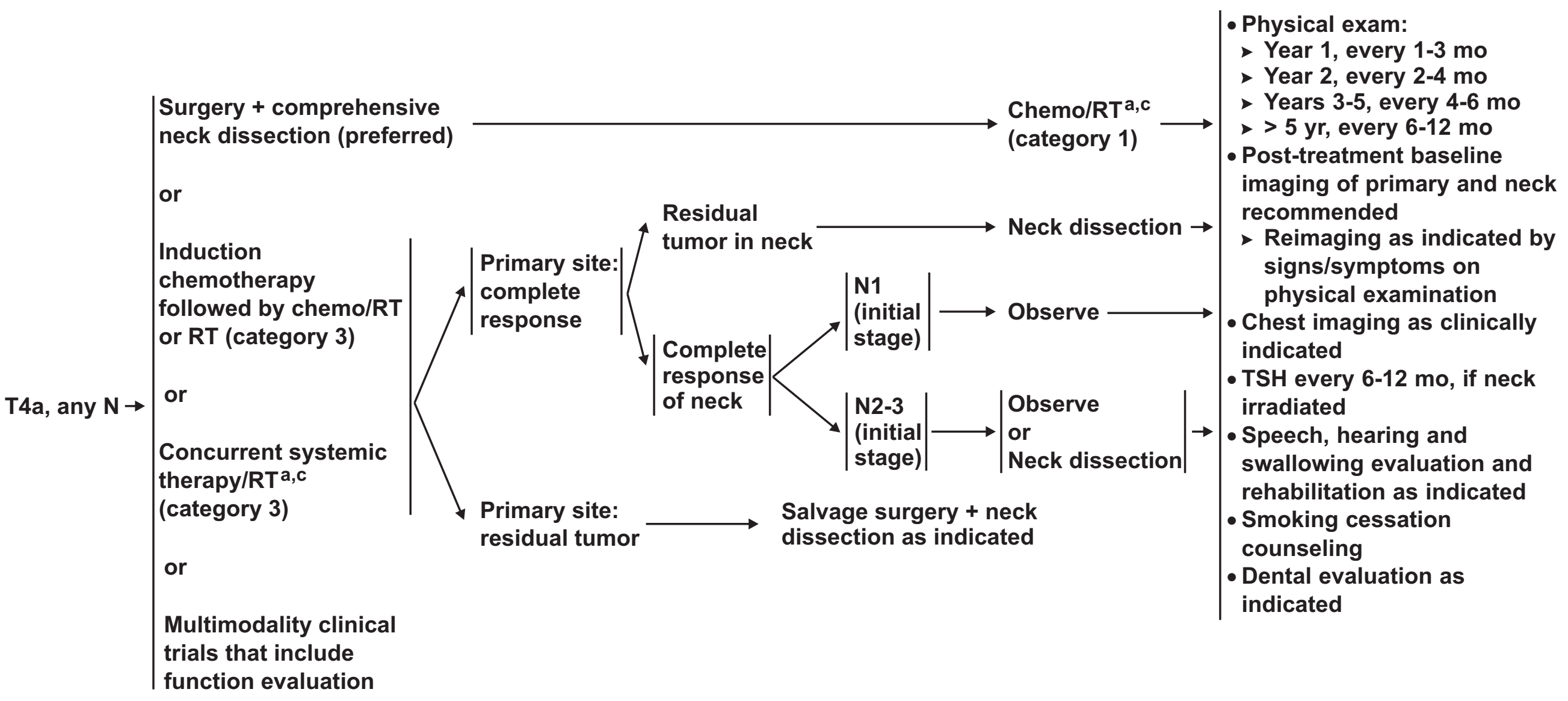

a See Principles of Radiation Therapy (HYPO-A).

c See Principles of Systemic Therapy (CHEM-A).

Note: All recommendations are category $2 \mathrm{~A}$ unless otherwise indicated.

Clinical Trials: NCCN believes that the best management of any cancer patient is in a clinical trial. Participation in clinical trials is especially encouraged. 


\section{PRINCIPLES OF RADIATION THERAPY}

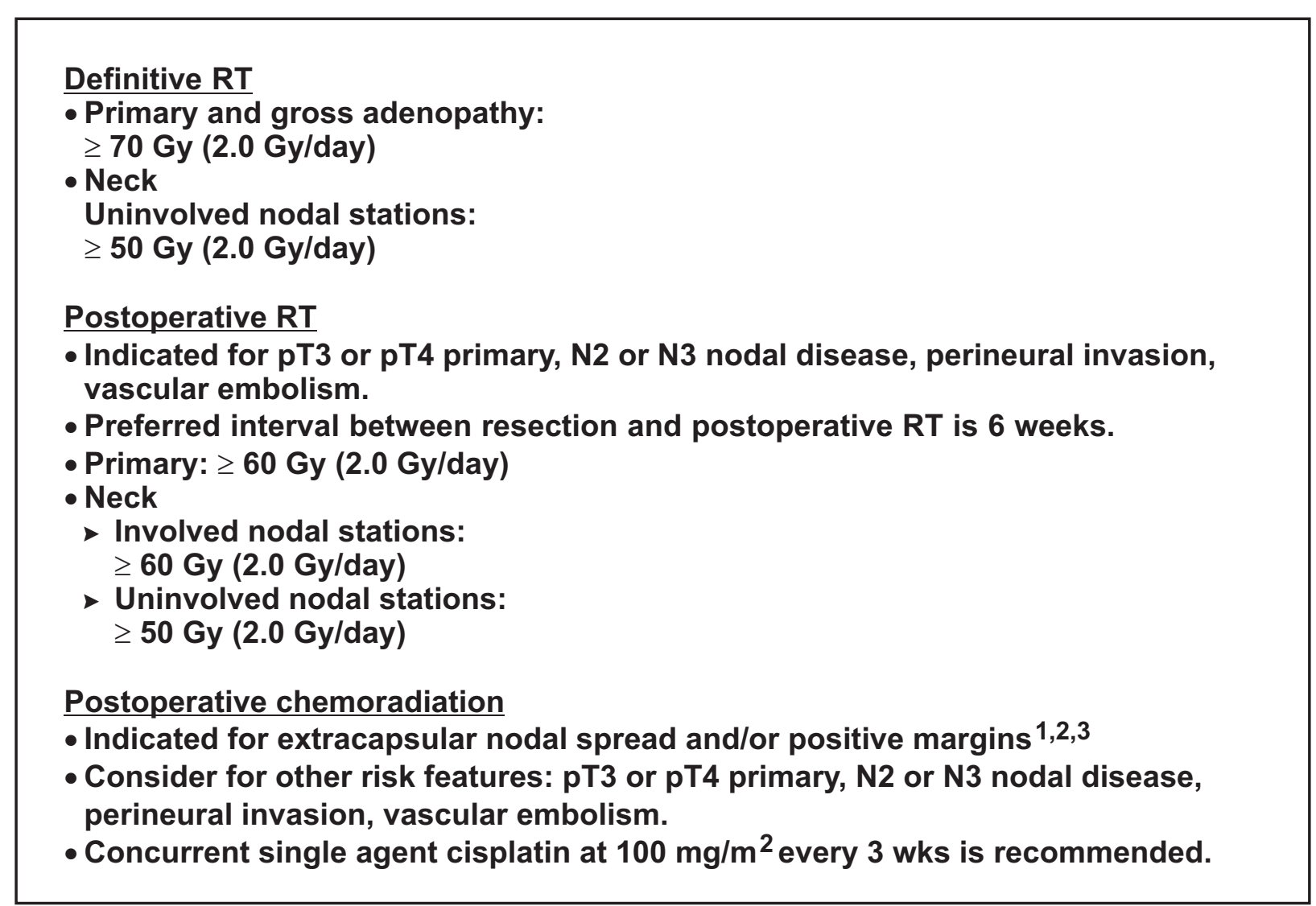

${ }^{1}$ Bernier J, Domenge C, Ozsahin M, et al. Postoperative irradiation with or without concomitant chemotherapy for locally advanced head and neck cancer. N Engl J Med 2004;350:1945-1952.

${ }^{2}$ Cooper JS, Pajak TF, Forastiere AA, et al. Postoperative concurrent radiotherapy and chemotherapy for high-risk squamous-cell carcinoma of the head and neck. N Engl J Med 2004;350:1937-1944.

${ }^{3}$ Bernier J, Cooper JS, Pajuk TF, et al. Defining risk levels in locally advanced head and neck cancers: A comparative analysis of concurrent postoperative radiation plus chemotherapy trials of the EORTC (\#22931) and RTOG (\#9501). Head Neck 2005;27:843-850. 


\section{PRESENTATION}

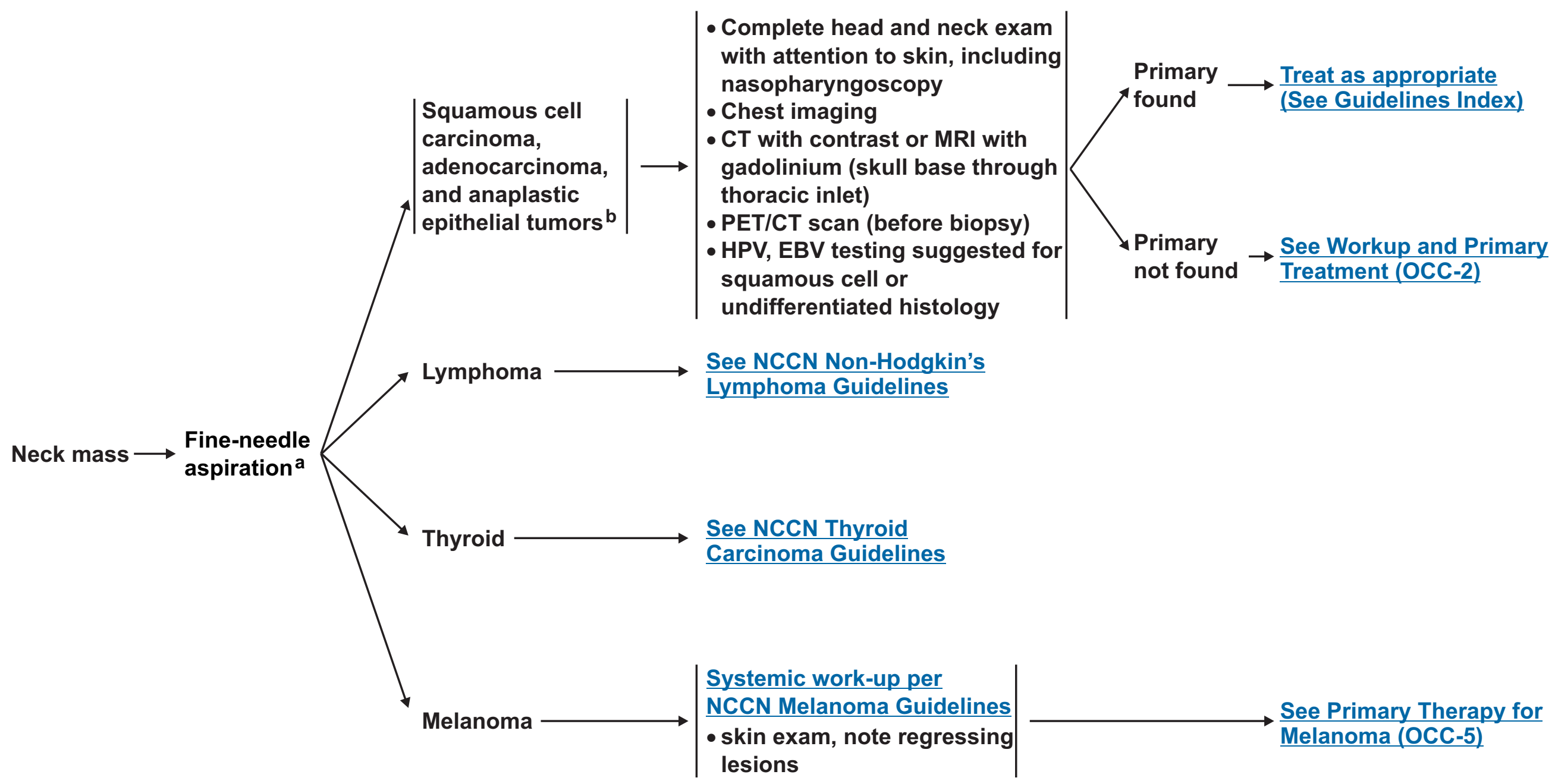

a Repeat FNA, core or open biopsy may be necessary for uncertain histologies. Patient should be prepared for neck dissection at time of biopsy, if necessary.

b Determined with appropriate immunohistochemical stains.

Note: All recommendations are category $2 \mathrm{~A}$ unless otherwise indicated.

Clinical Trials: NCCN believes that the best management of any cancer patient is in a clinical trial. Participation in clinical trials is especially encouraged. 


\section{PATHOLOGIC WORKUP FINDINGS}

- Examination under anesthesia

- Palpation and inspection

Node level

I, II, III,

upper $\mathbf{V}$

$\bullet$ clinical concern, including tonsillectomy - Direct laryngoscopy and nasopharynx survey

Node level
IV, lower V $\rightarrow\left|\begin{array}{l}\bullet \text { Direct laryngoscopy, } \\ \text { bronchoscopy, } \\ \text { esophagoscopy } \\ \bullet \text { Chest/abdominal/ } \\ \text { pelvic CT }\end{array}\right| \rightarrow$

' See Principles of Radiation Therapy (OCC-A) d See Principles of Systemic Therapy (CHEM-A).

\section{DEFINITIVE TREATMENT}

Treat as appropriate (See Guidelines Index) found Comprehensive

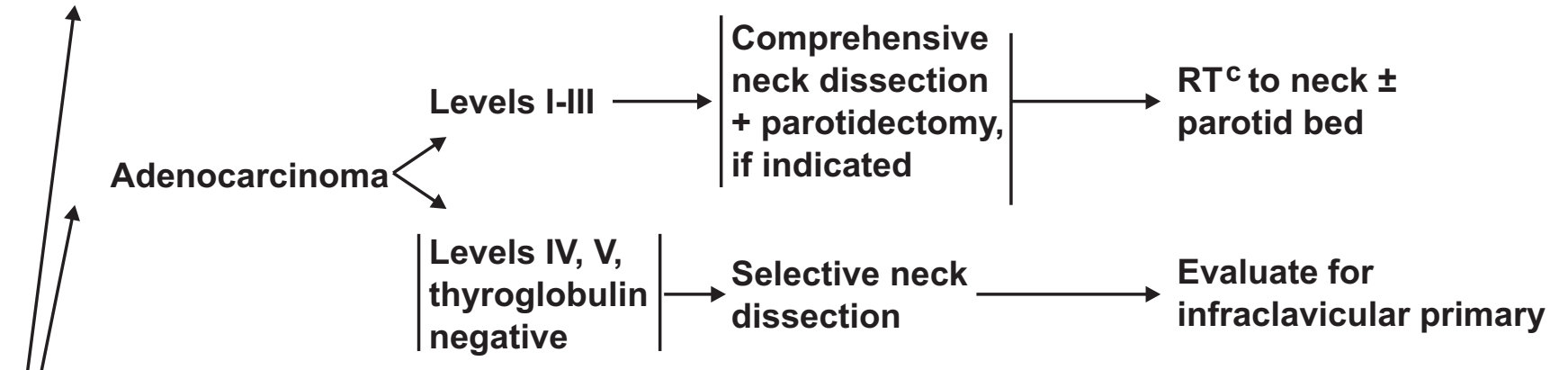

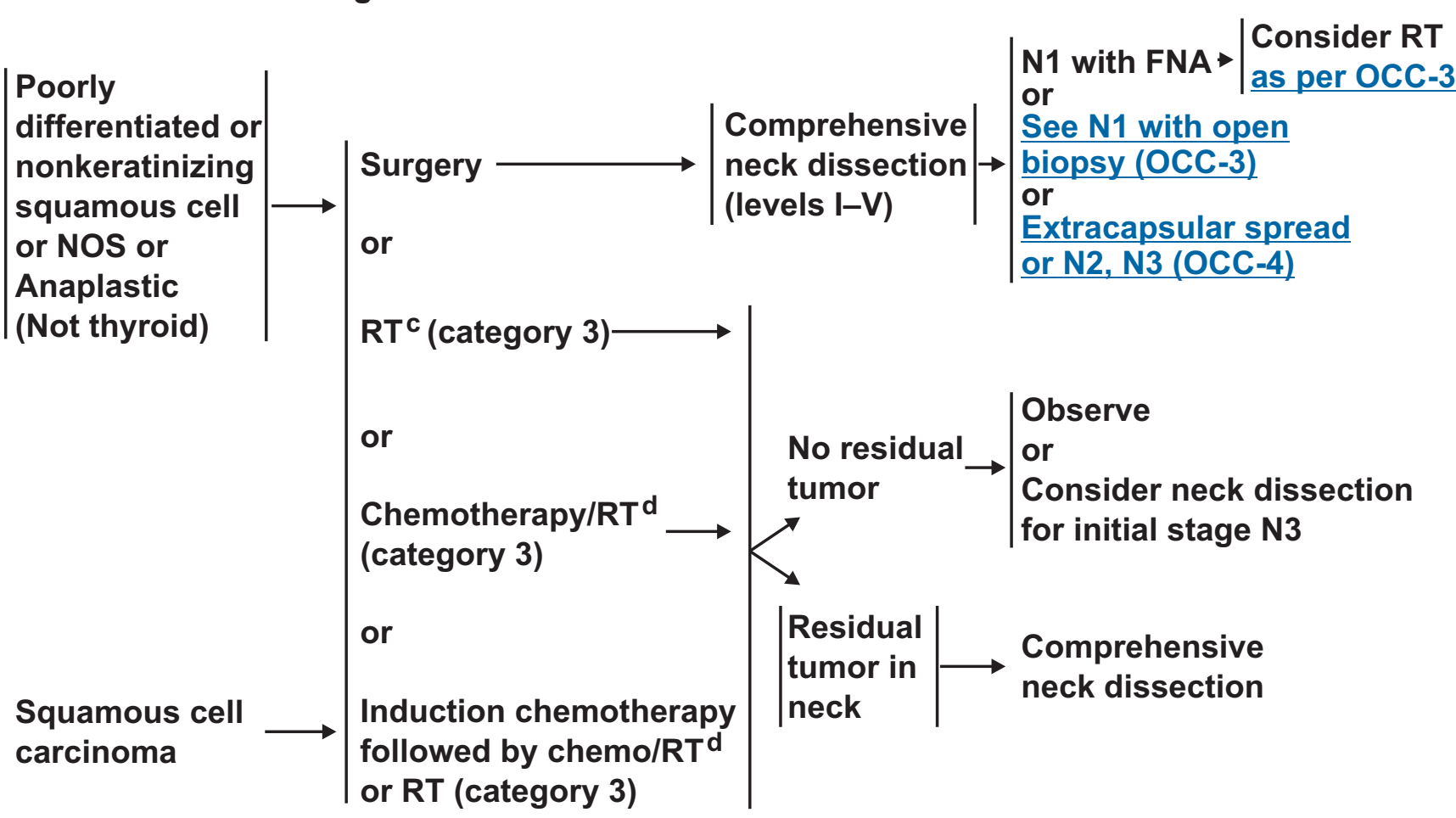

Note: All recommendations are category $2 \mathrm{~A}$ unless otherwise indicated.

Clinical Trials: NCCN believes that the best management of any cancer patient is in a clinical trial. Participation in clinical trials is especially encouraged. 


\section{POST NECK DISSECTION}

N1 with open biopsy

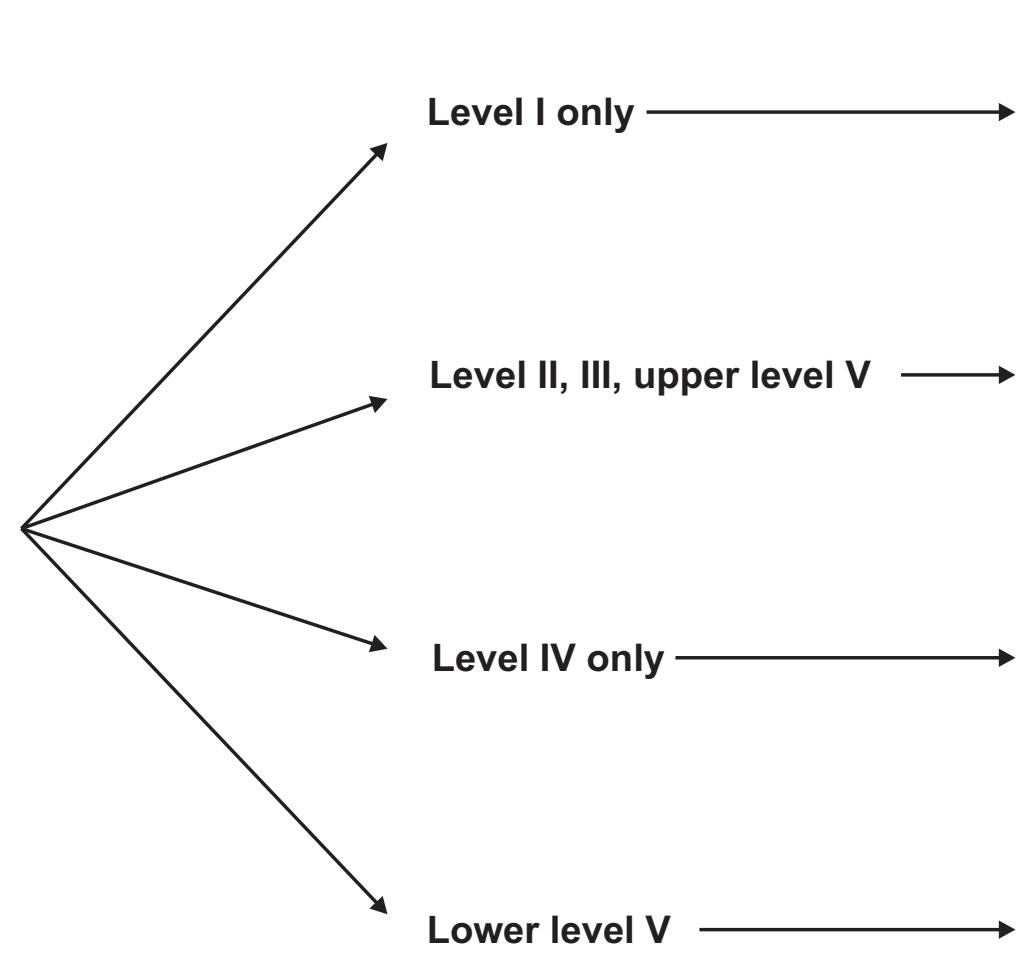

$\mathrm{RT}^{\mathrm{C}}$ to oral cavity, Waldeyer's ring, oropharynx, both sides of the neck (block RT to the larynx) or $\mathrm{RT}^{\mathrm{c}}$ to neck only (category 3 )

$\mathrm{RT}^{\mathrm{c}}$ to nasopharynx, both sides of the neck, hypopharynx, larynx, oropharynx

$\mathrm{RT}^{\mathrm{C}}$ to neck only (category 3 )

$\mathrm{RT}^{\mathrm{c}}$ to Waldeyer's ring, larynx, hypopharynx, both sides of the neck or $\mathrm{RT}^{\mathrm{c}}$ to neck only (category 3 )

$\mathrm{RT}^{\mathrm{C}}$ to larynx, hypopharynx, both sides of the neck or $\mathrm{RT}^{\mathrm{c}}$ to neck only (category 3 )

${ }^{\mathrm{C}}$ See Principles of Radiation Therapy (OCC-A).

Note: All recommendations are category 2A unless otherwise indicated.

Clinical Trials: NCCN believes that the best management of any cancer patient is in a clinical trial. Participation in clinical trials is especially encouraged. 


\section{POST NECK DISSECTION}

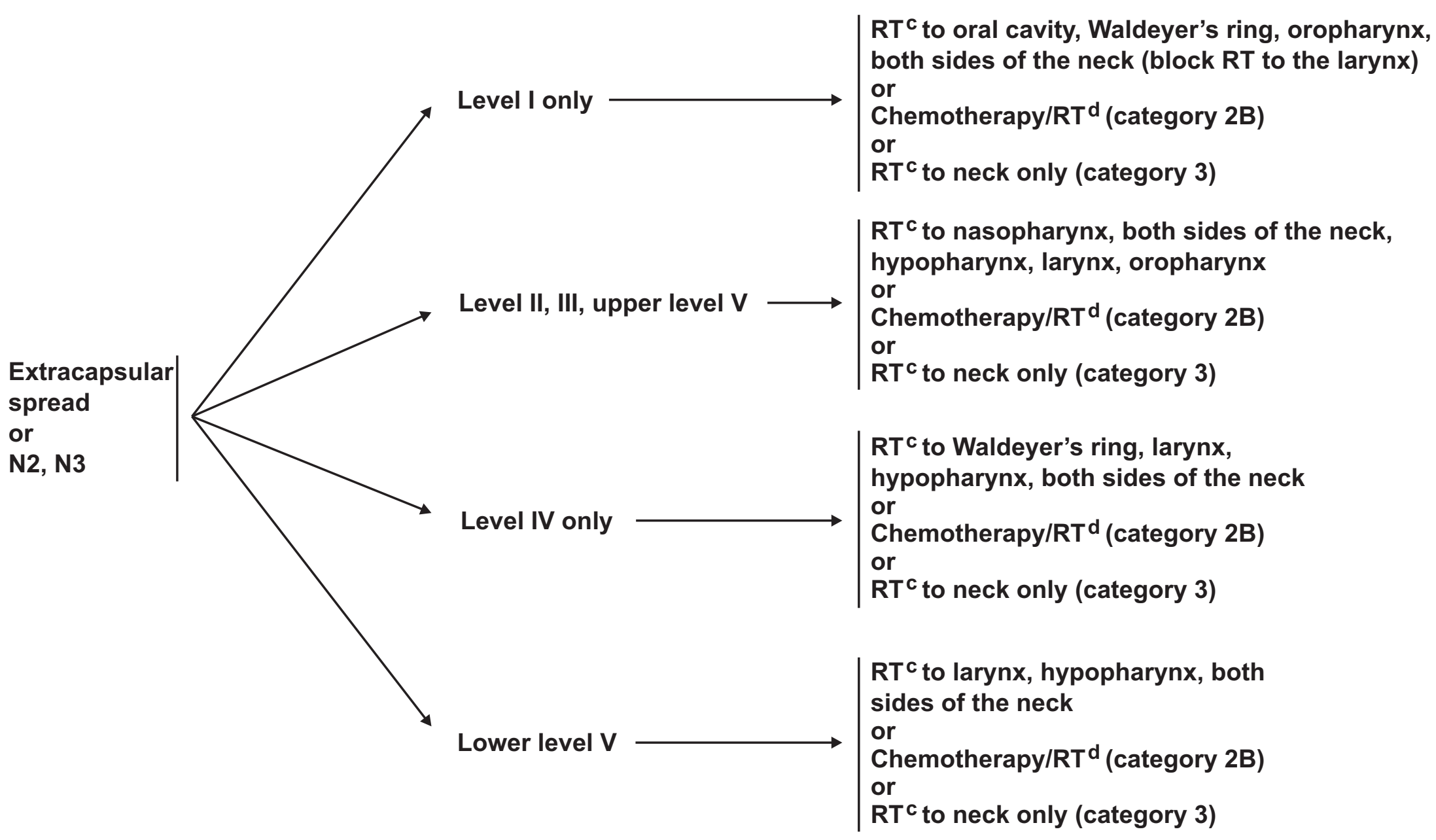

cSee Principles of Radiation Therapy (OCC-A).

$\mathrm{d}$ See Principles of Systemic Therapy (CHEM-A).

Note: All recommendations are category $2 \mathrm{~A}$ unless otherwise indicated.

Clinical Trials: NCCN believes that the best management of any cancer patient is in a clinical trial. Participation in clinical trials is especially encouraged. 


\section{$\mathrm{NCCN}^{\circ}$

\section{PRIMARY THERAPY FOR OCCULT PRIMARY- MELANOMA}

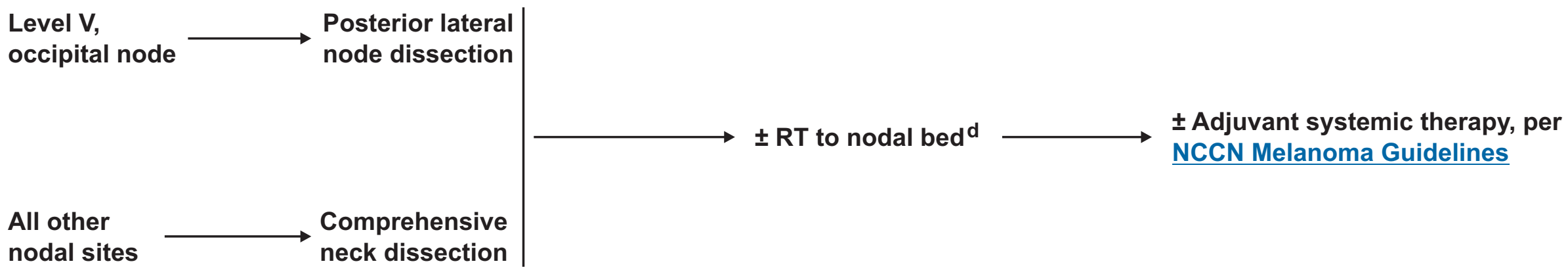

dAdjuvant radiotherapy: $30 \mathrm{~Gy} / 5 \mathrm{fx}$ over 2.5 weeks $(6.0 \mathrm{~Gy} / \mathrm{fx})$. Careful attention to dosimetry is necessary.

(Ang KK, Peters LJ, Weber RS, et al. Postoperative radiotherapy for cutaneous melanoma of the head and neck region.

International Journal of Radiation Oncology, Biology, Physics 30:795-798, 1994).

Note: All recommendations are category $2 \mathrm{~A}$ unless otherwise indicated.

Clinical Trials: NCCN believes that the best management of any cancer patient is in a clinical trial. Participation in clinical trials is especially encouraged. 


\section{PRINCIPLES OF RADIATION THERAPY}

\section{Mucosal sites:}

- 50-60 Gy (2.0 Gy/day) to mucosa, depending on field size and use of chemotherapy. Consider boost to 60-64 Gy to particularly suspicious areas

Neck

- Uninvolved nodal stations:

$\geq 50$ Gy (2.0 Gy/day)

- Involved nodal station(s):

60-66 Gy ${ }^{*}$ (2.0 Gy/day)

* Up to 70 Gy in case of excision only for N1 neck. 


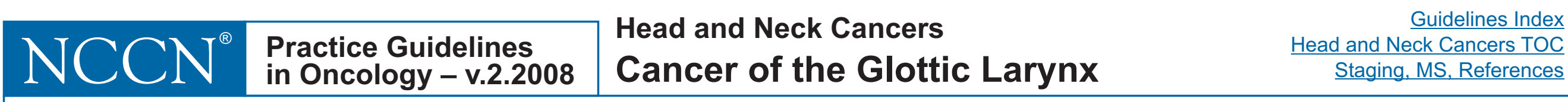

\section{WORKUPa}

CLINICAL STAGING

TREATMENT OF PRIMARY AND NECK

- H\&P

- Biopsy

- Chest imaging

- CT with contrast and thin cuts through larynx, or MRI of primary and neck recommended - Examination under anesthesia with endoscopy

- Preanesthesia studies

- Dental evaluation as indicated

- Speech \& swallowing evaluation as indicated

Multidisciplinary consultation as indicated

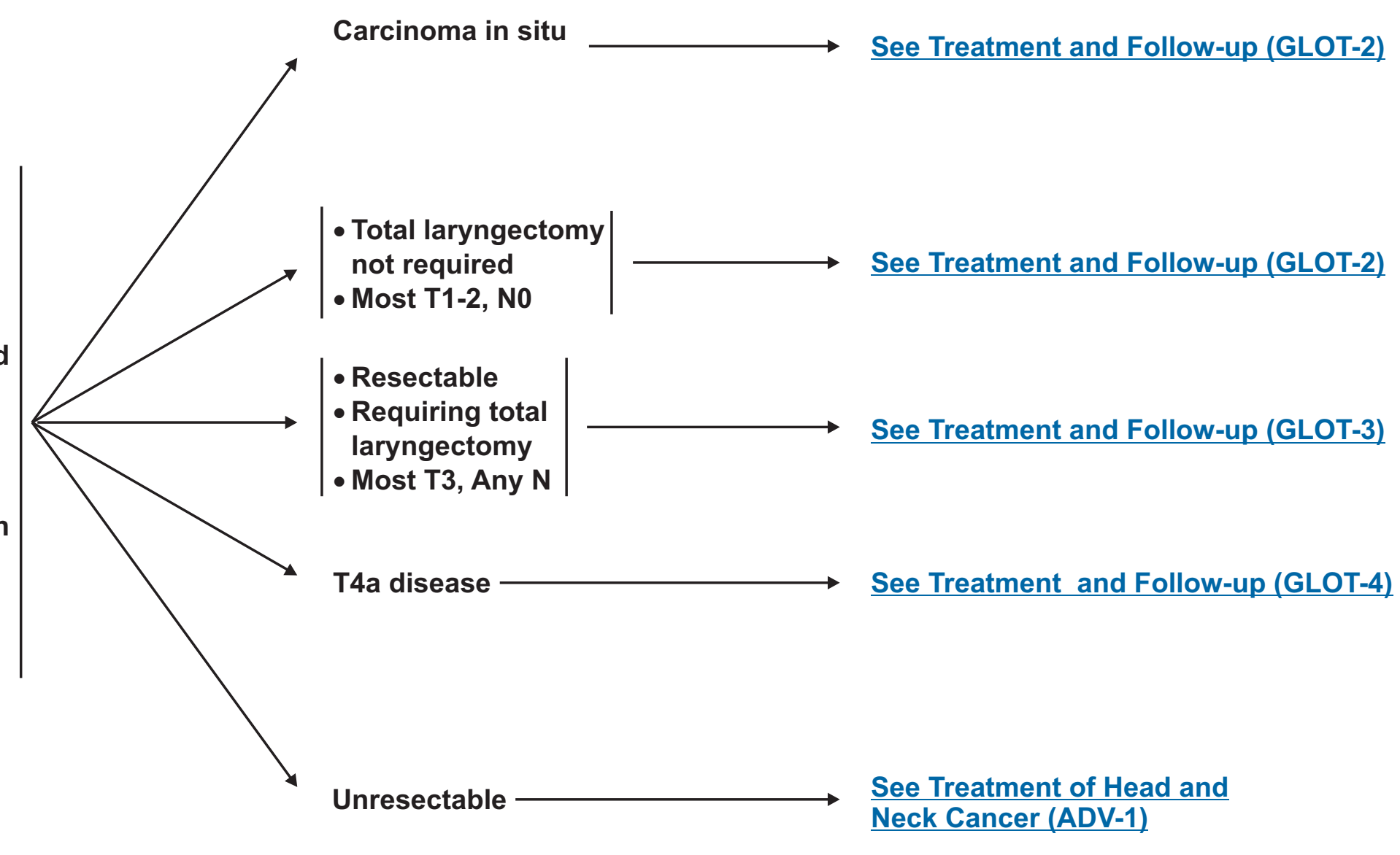

aComplete workup not indicated for Tis, T1 .

Note: All recommendations are category $2 \mathrm{~A}$ unless otherwise indicated.

Clinical Trials: NCCN believes that the best management of any cancer patient is in a clinical trial. Participation in clinical trials is especially encouraged. 


\section{$\mathrm{NCCN}^{\oplus}$ Practice Guidelines in Oncology - v.2.2008

\section{CLINICAL STAGING}

TREATMENT OF PRIMARY AND NECK

FOLLOW-UP
Carcinoma in situ

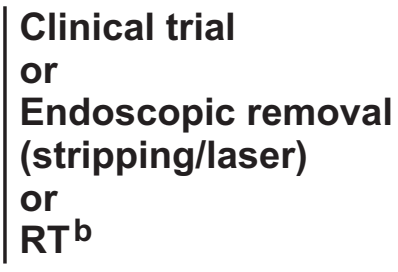

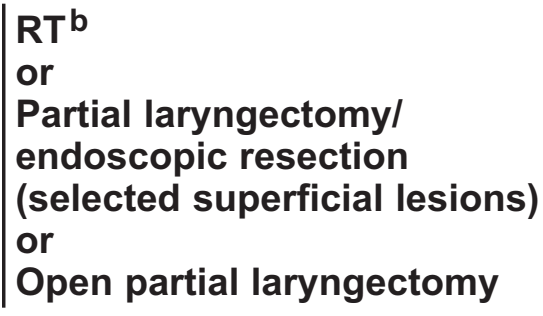

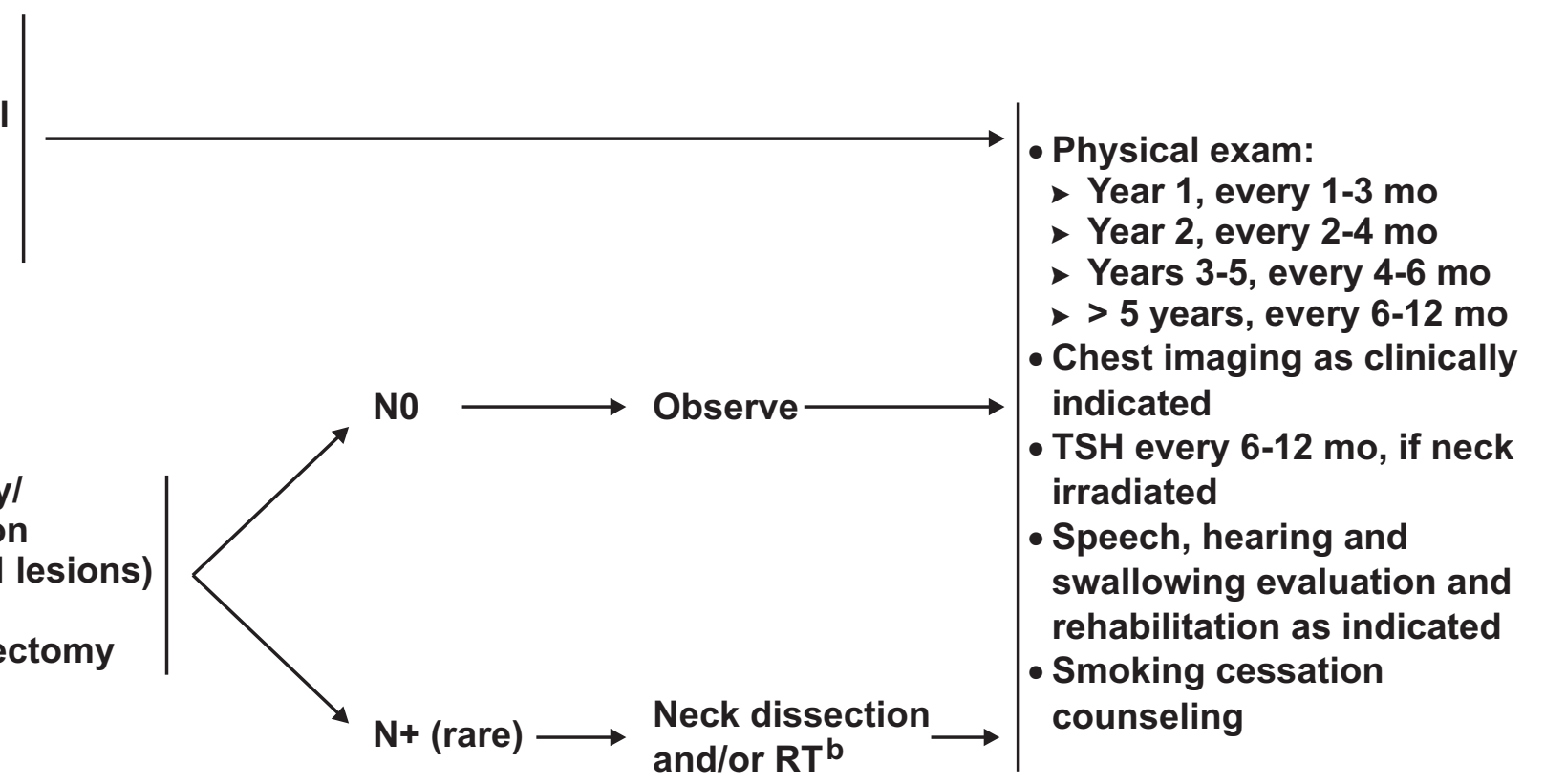

b See Principles of Radiation Therapy (GLOT-A).

Note: All recommendations are category $2 \mathrm{~A}$ unless otherwise indicated.

Clinical Trials: NCCN believes that the best management of any cancer patient is in a clinical trial. Participation in clinical trials is especially encouraged. 


\section{CLINICAL \\ STAGING \\ TREATMENT OF PRIMARY AND NECK}
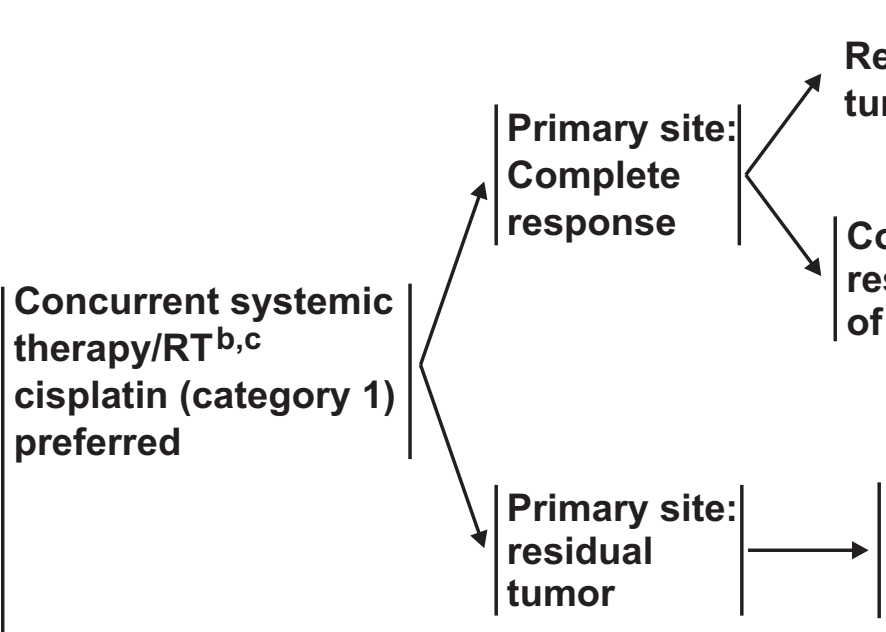

Residual

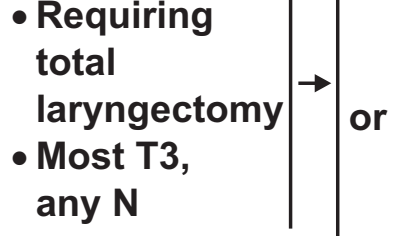

any $\mathbf{N}$

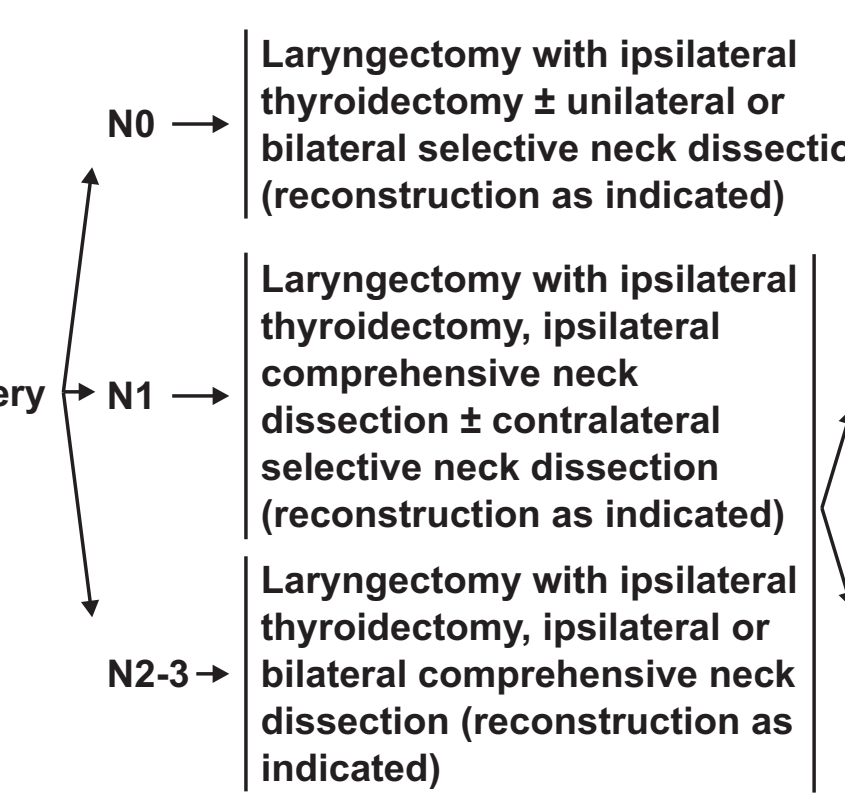

Salvage surgery

+ neck dissection

as indicated

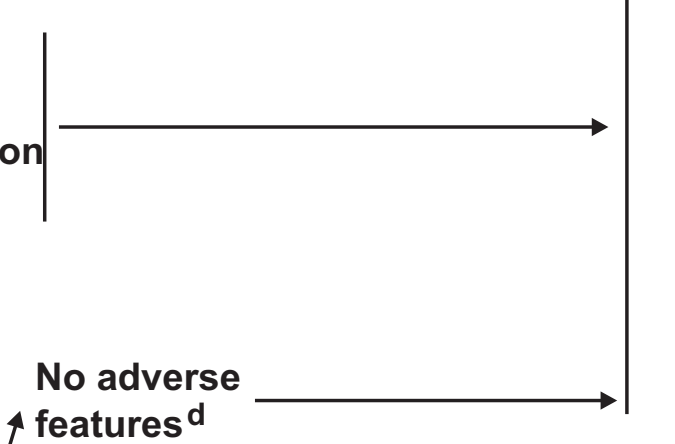
$\uparrow^{\text {features }}{ }^{d}$

\section{FOLLOW-UP} TREATMENT

Neck dissection
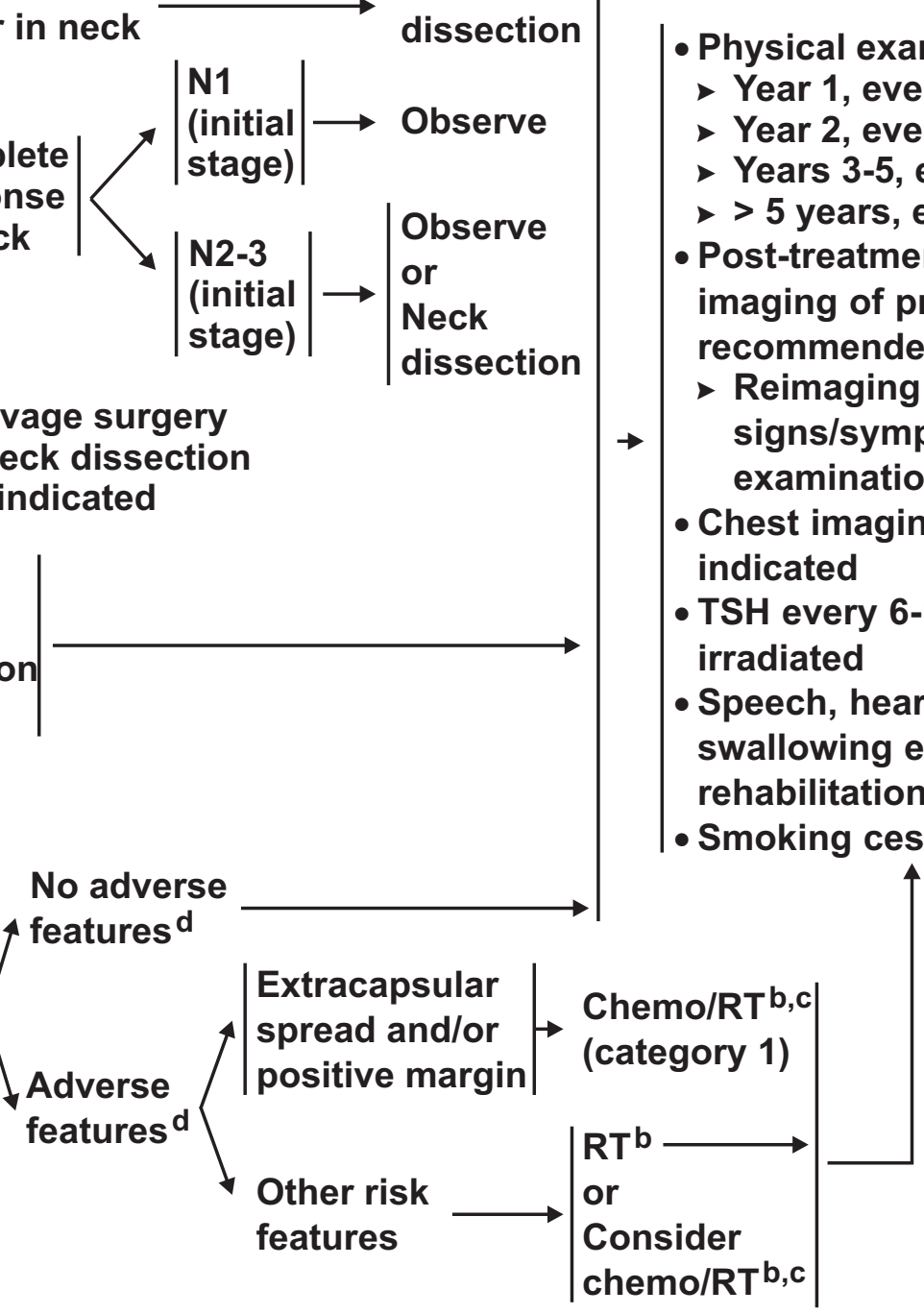

- Physical exam:

- Year 1, every 1-3 mo

- Year 2, every 2-4 mo

- Years 3-5, every 4-6 mo

$>5$ years, every 6-12 mo

- Post-treatment baseline imaging of primary and neck recommended

- Reimaging as indicated by signs/symptoms on physical examination

- Chest imaging as clinically indicated

- TSH every 6-12 mo, if neck irradiated

- Speech, hearing and swallowing evaluation and rehabilitation as indicated

- Smoking cessation counseling

b See Principles of Radiation Therapy (GLOT-A).

' See Principles of Systemic Therapy (CHEM-A).

$\mathrm{d}$ Risk features: extracapsular nodal spread, positive margins, pT4 primary, N2 or N3 nodal disease, perineural invasion, vascular embolism.

Note: All recommendations are category $2 \mathrm{~A}$ unless otherwise indicated.

Clinical Trials: NCCN believes that the best management of any cancer patient is in a clinical trial. Participation in clinical trials is especially encouraged. 


\section{CLINICAL \\ TREATMENT OF PRIMARY AND NECK \\ ADJUVANT \\ FOLLOW-UP \\ STAGING \\ TREATMENT}

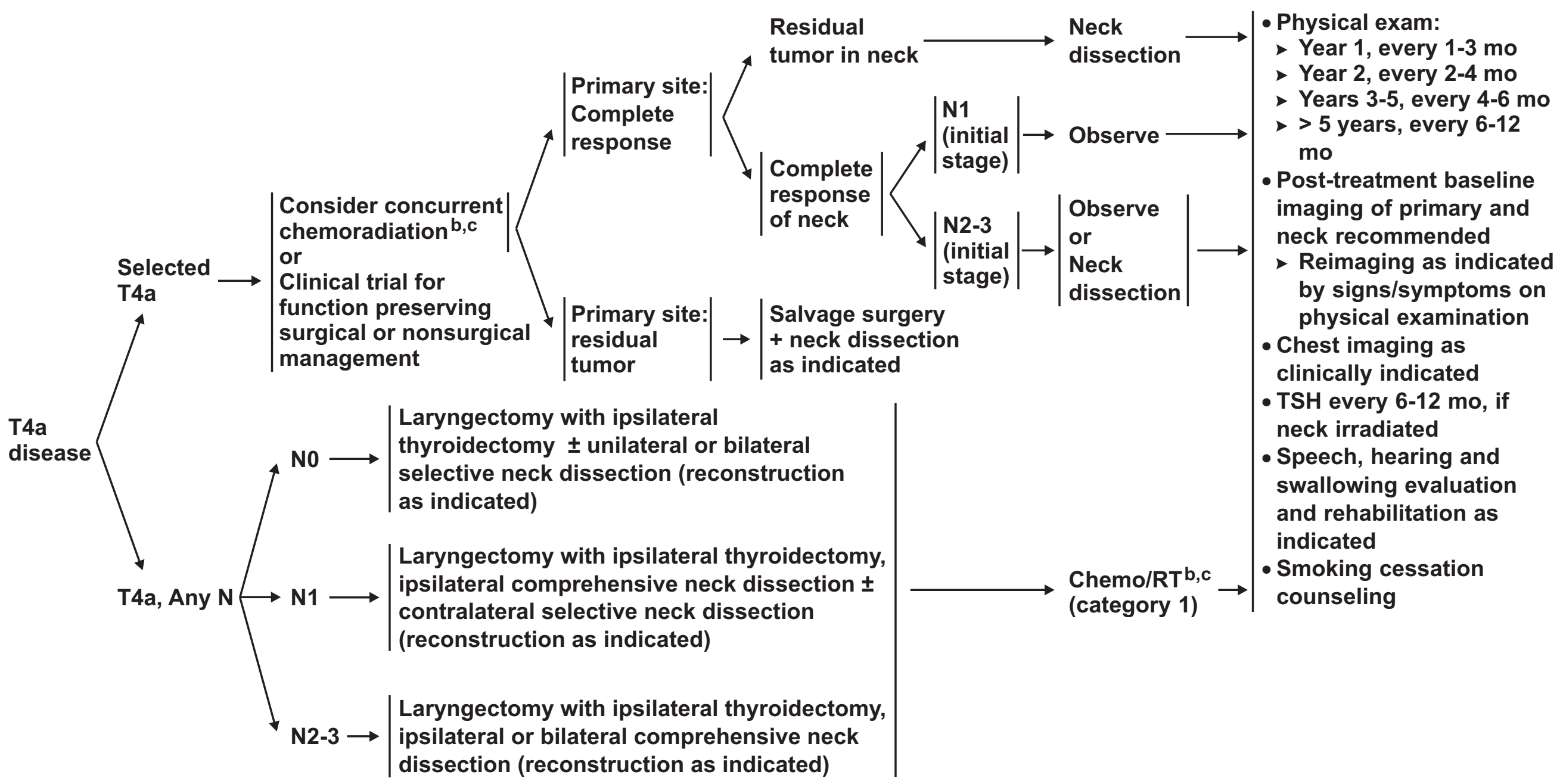

${ }^{\mathrm{b}}$ See Principles of Radiation Therapy (GLOT-A).

c See Principles of Systemic Therapy (CHEM-A).

Note: All recommendations are category $2 \mathrm{~A}$ unless otherwise indicated.

Clinical Trials: NCCN believes that the best management of any cancer patient is in a clinical trial. Participation in clinical trials is especially encouraged. 


\section{PRINCIPLES OF RADIATION THERAPY}

Definitive RT

- T1, N0: 63-66 Gy in 2.25-2.0 Gy/day

$\bullet \geq \mathrm{T} 2$ and gross adenopathy:

> $70 \mathrm{~Gy}(2.0 \mathrm{~Gy} / \mathrm{day})$ in 7 weeks

> $72 \mathrm{~Gy}$ in 6 weeks (1.8 Gy/fraction, large field; $1.5 \mathrm{~Gy}$ boost as second daily fraction during last 12 treatment days)

> 79.2 - 81.6 Gy in 7 weeks (1.2 Gy/fraction, twice daily)

- Elective nodal RT

> > 50 Gy (2.0 Gy/day)

Postoperative RT

- Indicated for PT4 primary, N2 or N3 nodal disease, perineural invasion, vascular embolism.

- Preferred interval between resection and postoperative RT is 6 weeks.

- Primary: $\geq 60$ Gy (2.0 Gy/day)

- Neck

- Involved nodal stations: $\geq 60$ Gy (2.0 Gy/day)

- Uninvolved nodal stations: $\geq 50$ Gy (2.0 Gy/day)

Postoperative chemoradiation

- Indicated for extracapsular nodal spread and/or positive margins $1,2,3$

- Consider for other risk features: pT4 primary, N2 or N3 nodal disease, perineural invasion, vascular embolism

- Concurrent single agent cisplatin at $100 \mathrm{mg} / \mathrm{m}^{2}$ every 3 wks is recommended.

${ }^{1}$ Bernier J, Domenge C, Ozsahin M, et al. Postoperative irradiation with or without concomitant chemotherapy for locally advanced head and neck cancer. N Engl J Med 2004;350:1945-1952.

${ }^{2}$ Cooper JS, Pajak TF, Forastiere AA, et al. Postoperative concurrent radiotherapy and chemotherapy for high-risk squamous-cell carcinoma of the head and neck. N Engl J Med 2004;350:1937-1944.

${ }^{3}$ Bernier J, Cooper JS, Pajuk TF, et al. Defining risk levels in locally advanced head and neck cancers: A comparative analysis of concurrent postoperative radiation plus chemotherapy trials of the EORTC (\#22931) and RTOG (\#9501). Head Neck 2005;27:843-850.

Note: All recommendations are category $2 \mathrm{~A}$ unless otherwise indicated.

Clinical Trials: NCCN believes that the best management of any cancer patient is in a clinical trial. Participation in clinical trials is especially encouraged. 


\section{$\mathrm{NCCN}^{\oplus}$ Practice Guidelines

\section{WORKUP}

- H\&P

- Biopsy

- Chest imaging

- CT with contrast and thin cuts through larynx or MRI of primary and neck recommended

- Examination under anesthesia with endoscopy

- Preanesthesia studies

- Dental evaluation as indicated

- Speech \& swallowing evaluation as indicated

Multidisciplinary consultation as indicated

\section{CLINICAL STAGING}

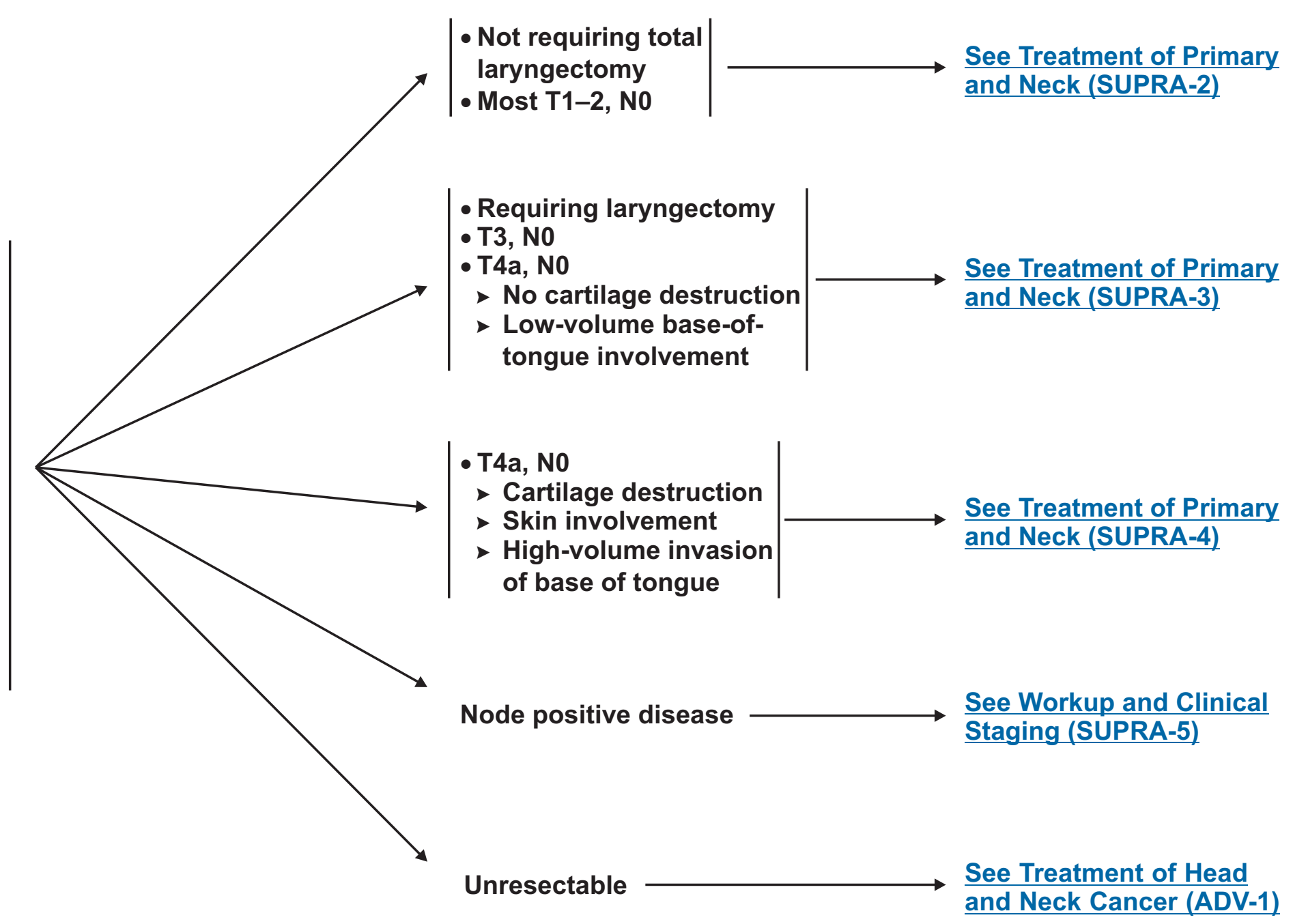

Note: All recommendations are category $2 \mathrm{~A}$ unless otherwise indicated.

Clinical Trials: NCCN believes that the best management of any cancer patient is in a clinical trial. Participation in clinical trials is especially encouraged. 


\section{$\mathrm{NCCN}{ }^{\circledast}$ Practice Guidelines in Oncology - v.2.2008 \\ Head and Neck Cancers \\ Cancer of the Supraglottic Larynx}

ADJUVANT

TREATMENT

\section{CLINICAL STAGING}

\section{TREATMENT OF PRIMARY AND NECK}

FOLLOW-UP
- Not requiring total

laryngectomy

- Most T1-2, NO

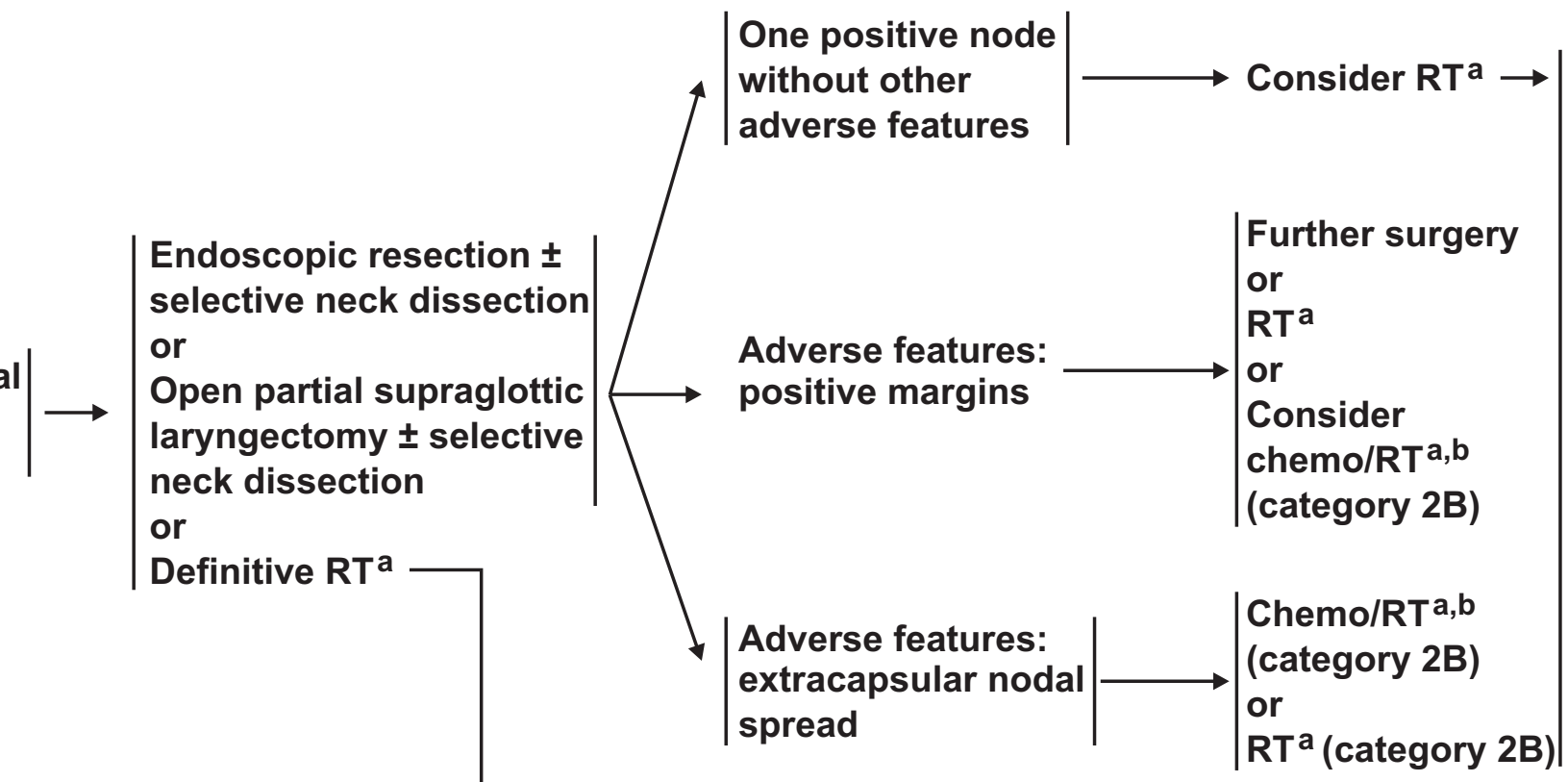

- Physical exam:

- Year 1 , every 1-3 mo

- Year 2, every 2-4 mo

- Years 3-5, every 4-6 mo

> 5 years, every 6-12 mo

- Chest imaging as clinically indicated

- TSH every 6-12 mo, if neck irradiated

- Speech, hearing and swallowing evaluation and rehabilitation as indicated

- Smoking cessation counseling

a See Principles of Radiation Therapy (SUPRA-A).

b See Principles of Systemic Therapy (CHEM-A).

Note: All recommendations are category $2 \mathrm{~A}$ unless otherwise indicated.

Clinical Trials: NCCN believes that the best management of any cancer patient is in a clinical trial. Participation in clinical trials is especially encouraged. 


\section{CLINICAL STAGING}

- Requiring

laryngectomy

- T3, NO

- T4a, No

- No cartilage destruction

- Low-volume base-oftongue involvement
TREATMENT OF PRIMARY AND NECK
ADJUVANT
TREATMENT

FOLLOW-UP
NO or one positive node

Laryngectomy, ipsilateral thyroidectomy with ipsilateral or bilateral selective neck dissection

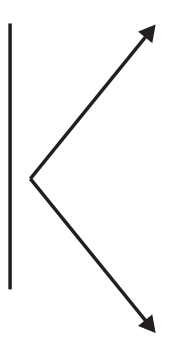

$\rightarrow$ or

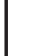
Concurrent systemic
therapy/RTa,b
cisplatin (category 1)
preferred Primary si
residual
tumor

without adverse features ${ }^{c}$

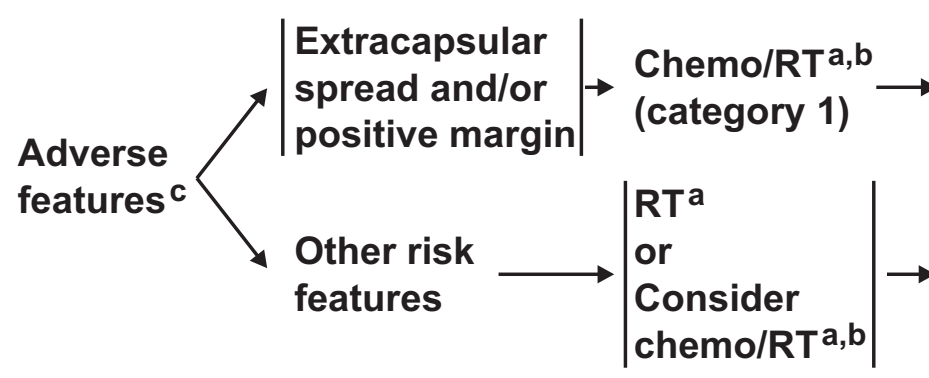

\section{Primary site:}

Complete response
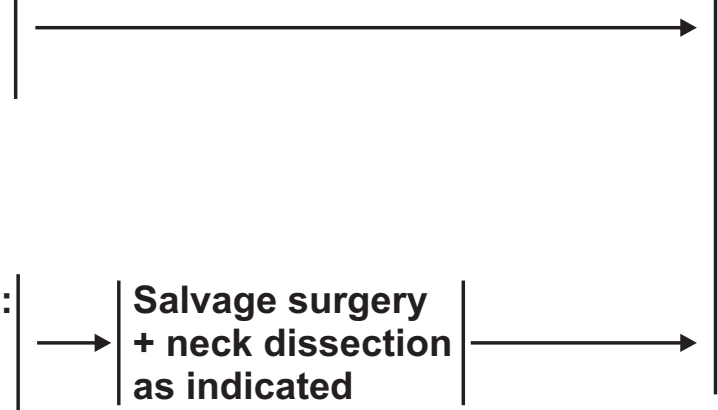

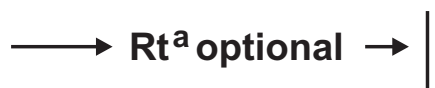

- Physical exam:

- Year 1, every 1-3 mo

- Year 2, every 2-4 mo

- Years 3-5, every 4-6 mo

> 5 years, every 6-12 mo

- Post-treatment baseline imaging of primary and neck recommended

- Reimaging as indicated by signs/symptoms on physical examination

- Chest imaging as clinically indicated

- TSH every 6-12 mo, if neck irradiated

- Speech, hearing and swallowing evaluation and rehabilitation as indicated

- Smoking cessation counseling

a See Principles of Radiation Therapy (SUPRA-A).

b See Principles of Systemic Therapy (CHEM-A).

${ }^{\mathrm{c}}$ Risk features: extracapsular nodal spread, positive margins, pT4 primary, N2 or N3 nodal disease, perineural invasion, vascular embolism.

Note: All recommendations are category $2 \mathrm{~A}$ unless otherwise indicated.

Clinical Trials: NCCN believes that the best management of any cancer patient is in a clinical trial. Participation in clinical trials is especially encouraged. 


\section{NCCN ${ }^{\oplus}$ Practice Guidelines

\section{CLINICAL STAGING}

\section{TREATMENT OF PRIMARY AND NECK}

ADJUVANT

TREATMENT
FOLLOW-UP
- T4a, No

- Cartilage destruction

- Skin involvement

- High-volume invasion of base of tongue
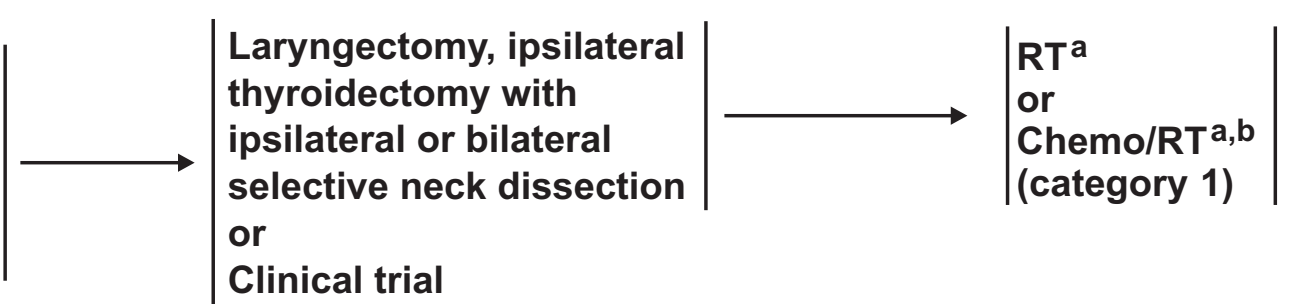

Clinical trial
- Physical exam:

- Year 1 , every 1-3 mo

- Year 2, every 2-4 mo

- Years 3-5, every 4-6 mo

> $>5$ years, every 6-12 mo

- Post-treatment baseline imaging of primary and neck recommended

- Reimaging as indicated by signs/symptoms on physical examination

- Chest imaging as clinically indicated

- TSH every 6-12 mo, if neck irradiated

- Speech, hearing and swallowing evaluation and rehabilitation as indicated

- Smoking cessation counseling

Recurrence (see ADV-2)

a See Principles of Radiation Therapy (SUPRA-A).

b See Principles of Systemic Therapy (CHEM-A).

Note: All recommendations are category $2 \mathrm{~A}$ unless otherwise indicated.

Clinical Trials: NCCN believes that the best management of any cancer patient is in a clinical trial. Participation in clinical trials is especially encouraged. 


\section{WORKUP}

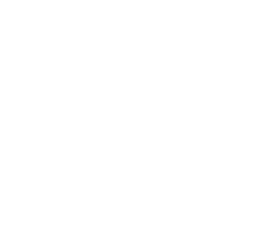

Node positive disease
- H\&P

- Biopsy

- Chest imaging

- CT with contrast and thin

cuts through larynx

- MRI of primary and neck

recommended

- Examination under anesthesia

with endoscopy

- Preanesthesia studies

- Dental evaluation as indicated

- Speech \& swallowing

evaluation as indicated

Multidisciplinary

consultation as indicated

\section{CLINICAL STAGING}

- Not requiring total

laryngectomy

- T1-2, N+ and

selected T3-4a

See Treatment of Primary and Neck (SUPRA-6)

See Treatment of Primary and Neck (SUPRA-7)

- Most T3-4a, N+

- No cartilage destruction

- Low-volume base-oftongue involvement

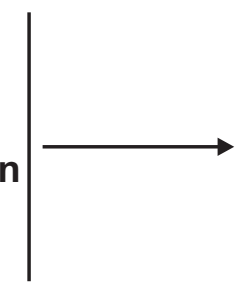

See Treatment of Primary and Neck (SUPRA-8)

- Cartilage destruction

- Skin involvement

- High-volume invasion of base of tongue

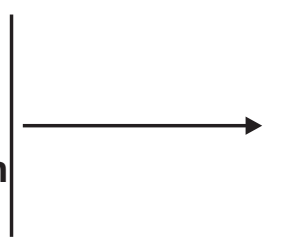

See Treatment of Head and

Unresectable (T4b) Neck Cancer (ADV-1)

Note: All recommendations are category $2 \mathrm{~A}$ unless otherwise indicated.

Clinical Trials: NCCN believes that the best management of any cancer patient is in a clinical trial. Participation in clinical trials is especially encouraged. 


\section{$\mathrm{NCCN}^{\oplus}$ Practice Guidelines

\section{CLINICAL \\ TREATMENT OF PRIMARY AND NECK} STAGING$$
\text { | }
$$

laryngectomy

- T1-2, N+ and selected

T3-4a

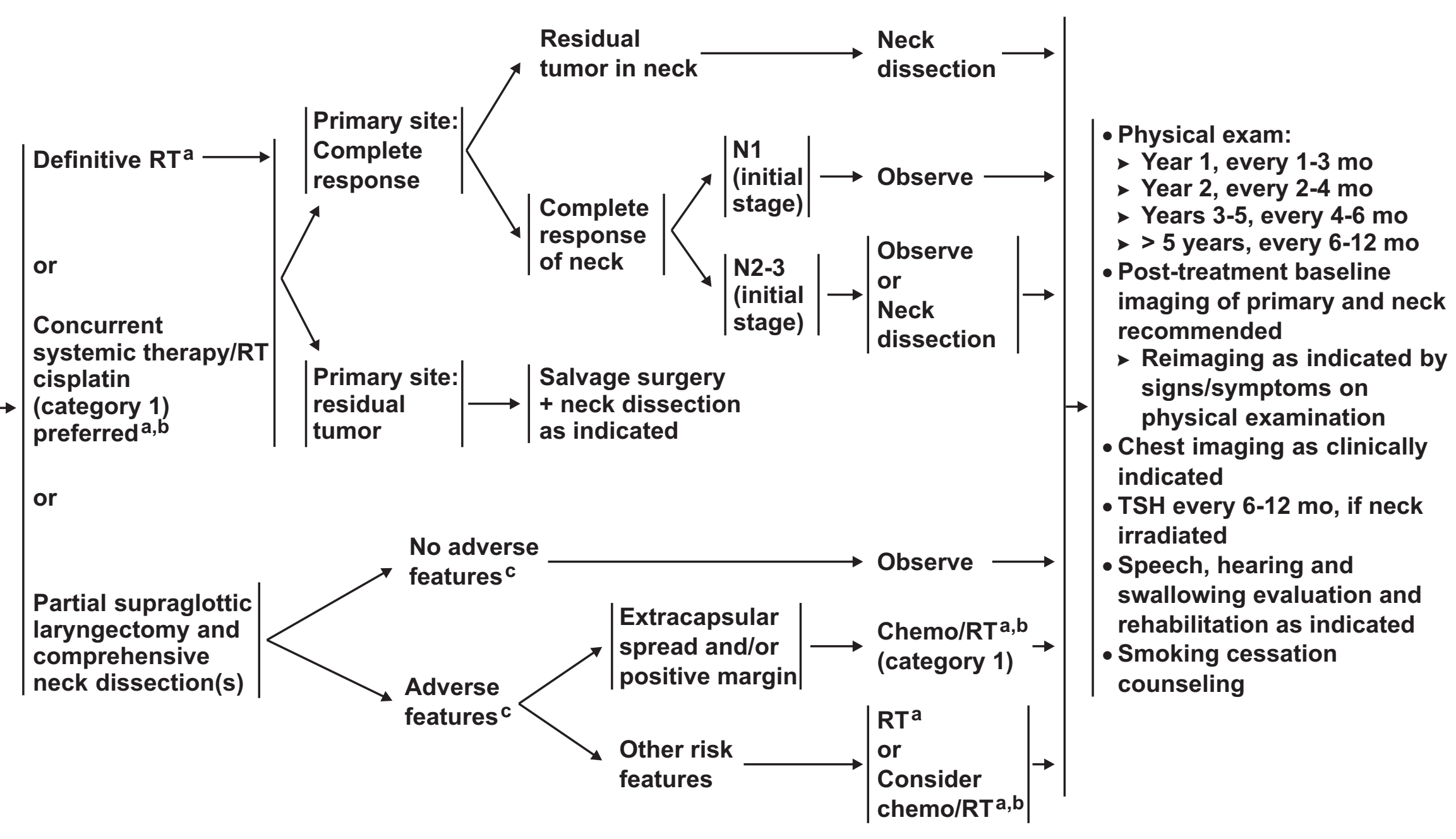

Recurrence (see ADV-2)

Year 2 every 2-4 mo

- Years 3-5, every 4-6 mo

6ears, every 6-12 mo

imaging of primary and neck mended signs/symptoms on indicated

TSH every 6-12 mo, if neck irradiated

Speech, hearing and renatitation as indicated oking cessation

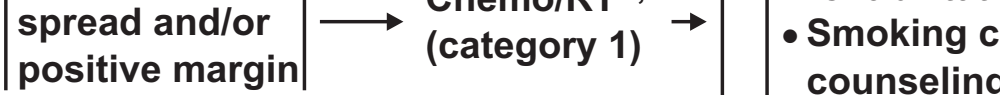

a See Principles of Radiation Therapy (SUPRA-A).

ADJUVANT FOLLOW-UP TREATMENT

bee Principles of Systemic Therapy (CHEM-A).

${ }^{c}$ Risk features: extracapsular nodal spread, positive margins, pT4 primary, N2 or N3 nodal disease, perineural invasion, vascular embolism.

Note: All recommendations are category $2 \mathrm{~A}$ unless otherwise indicated.

Clinical Trials: NCCN believes that the best management of any cancer patient is in a clinical trial. Participation in clinical trials is especially encouraged. 


\section{CLINICAL STAGING TREATMENT OF PRIMARY AND NECK}

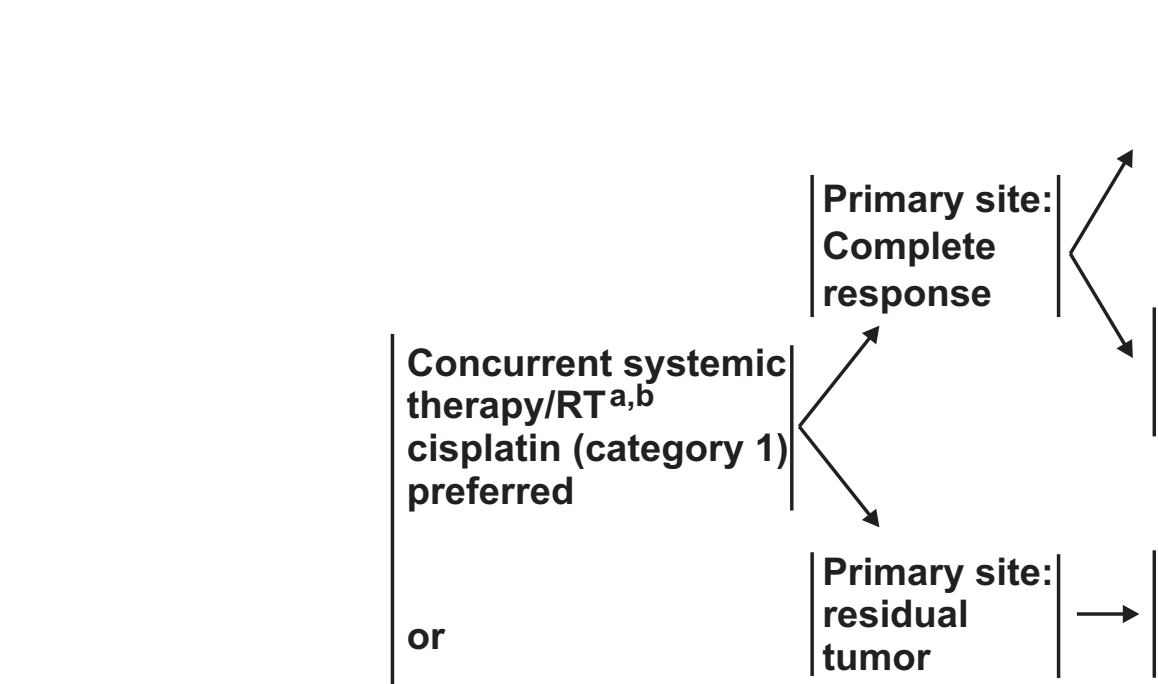

- Requiring total laryngectomy

- Most T3-4a, N+

- No cartilage destruction

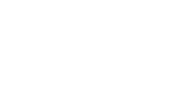

Adverse

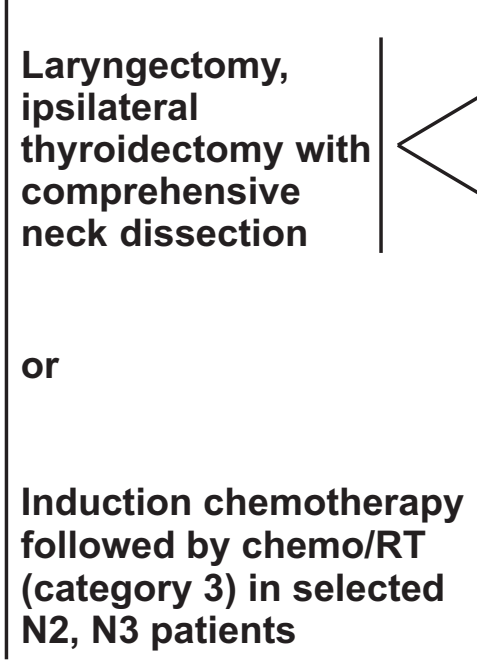

Induction chemotherapy followed by chemo/RT

(category 3 ) in selected

N2, N3 patients features ${ }^{c}$

Complete response of neck

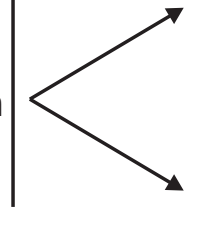
Salvage surgery +
neck dissection as indicated

Residual tumor in neck
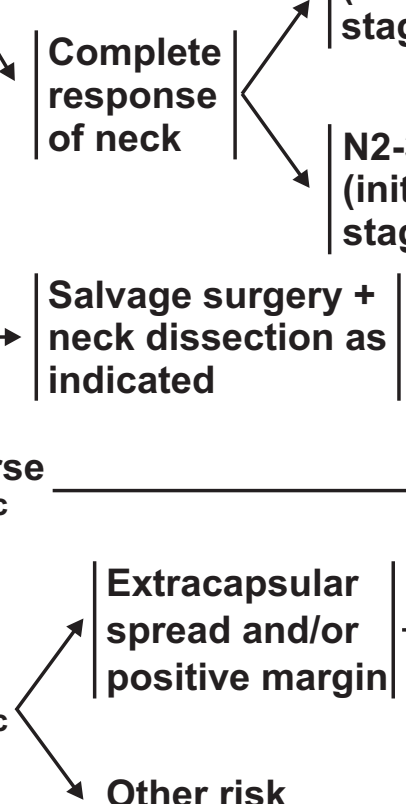

\section{Extracapsular} spread and/or positive margin

Other risk features

ADJUVANT TREATMENT

Neck dissection

\section{FOLLOW-UP}

- Physical exam:

- Year 1, every 1-3 mo

- Year 2, every 2-4 mo

- Years 3-5, every 4-6 mo

$>$ years, every 6-12 mo

- Post-treatment baseline imaging of primary and neck recommended

> Reimaging as indicated by signs/symptoms on physical examination

- Chest imaging as clinically indicated

- TSH every 6-12 mo, if neck irradiated

- Speech, hearing and swallowing evaluation and rehabilitation as indicated

- Smoking cessation counseling

a See Principles of Radiation Therapy (SUPRA-A).

Recurrence (see ADV-2)

${ }^{b}$ See Principles of Systemic Therapy (CHEM-A).

${ }^{c}$ Risk features: extracapsular nodal spread, positive margins, pT4 primary, N2 or N3 nodal disease, perineural invasion, vascular embolism.

Note: All recommendations are category $2 \mathrm{~A}$ unless otherwise indicated.

Clinical Trials: NCCN believes that the best management of any cancer patient is in a clinical trial. Participation in clinical trials is especially encouraged. 


\section{NCCN ${ }^{\oplus}$ Practice Guidelines

TREATMENT OF

PRIMARY AND NECK

ADJUVANT

TREATMENT

\section{FOLLOW-UP}

$\mathrm{T} 4 \mathrm{a}, \mathrm{N}+$

- Cartilage destruction

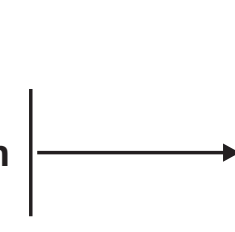

Laryngectomy, ipsilateral thyroidectomy with ipsilateral or bilateral neck dissection or

Clinical trial
- Physical exam:

- Year 1, every 1-3 mo

- Year 2, every 2-4 mo

- Years 3-5, every 4-6 mo

> 5 years, every 6-12 mo

- Post-treatment baseline imaging of primary

and neck recommended

- Reimaging as indicated by signs/symptoms on physical examination - Chest imaging as clinically indicated

- TSH every 6-12 mo, if neck irradiated

- Speech, hearing and swallowing evaluation and rehabilitation as indicated

- Smoking cessation counseling

Note: All recommendations are category $2 \mathrm{~A}$ unless otherwise indicated.

Clinical Trials: NCCN believes that the best management of any cancer patient is in a clinical trial. Participation in clinical trials is especially encouraged. 


\section{PRINCIPLES OF RADIATION THERAPY}

Definitive RT

- Primary and gross adenopathy:

$\geq 70$ Gy (2.0 Gy/day)

- Neck

- Uninvolved nodal stations:

$\geq 50$ Gy (2.0 Gy/day)

\section{Postoperative RT}

- Indicated for pT4 primary, N2 or N3 nodal disease, perineural invasion, vascular embolism.

- Preferred interval between resection and postoperative RT is 6 weeks.

- Primary: $\geq 60$ Gy (2.0 Gy/day)

- Neck

- Involved nodal stations:

$\geq 60$ Gy (2.0 Gy/day)

- Uninvolved nodal stations:

$\geq 50$ Gy (2.0 Gy/day)

Postoperative chemoradiation

- Indicated for extracapsular nodal spread and/or positive margins ${ }^{1,2,3}$

- Consider for other risk features: pT4 primary, N2 or N3 nodal disease, perineural

invasion, vascular embolism.

- Concurrent single agent cisplatin at $100 \mathrm{mg} / \mathrm{m}^{2}$ every 3 wks is recommended.

${ }^{1}$ Bernier J, Domenge C, Ozsahin M, et al. Postoperative irradiation with or without concomitant chemotherapy for locally advanced head and neck cancer. N Engl J Med 2004;350:1945-1952.

${ }^{2}$ Cooper JS, Pajak TF, Forastiere AA, et al. Postoperative concurrent radiotherapy and chemotherapy for high-risk squamous-cell carcinoma of the head and neck. N Engl J Med 2004;350:1937-1944.

${ }^{3}$ Bernier J, Cooper JS, Pajuk TF, et al. Defining risk levels in locally advanced head and neck cancers: A comparative analysis of concurrent postoperative radiation plus chemotherapy trials of the EORTC (\#22931) and RTOG (\#9501). Head Neck 2005;27:843-850. Node positive (SUPRA-5)

Note: All recommendations are category $2 \mathrm{~A}$ unless otherwise indicated.

Clinical Trials: NCCN believes that the best management of any cancer patient is in a clinical trial. Participation in clinical trials is especially encouraged. 


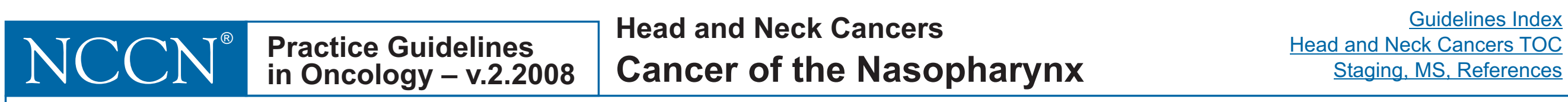

\section{WORKUP}

$\bullet H \& P$

- Nasopharyngeal exam and biopsy

- Chest imaging

- MRI with gadolinium of

nasopharynx and base of skull to

clavicles and/or CT with contrast

- Dental evaluation as indicated

- Speech \& swallowing evaluation

as indicated

- Imaging for distant metastases

(chest, liver, bone) for WHO class

2-3/N2-3 disease (may include

PET scan and/or CT)

Multidisciplinary consultation
CLINICAL STAGING

T1, NO, MO and

T2a, NO, MO

See Treatment of Primary and Neck (NASO-2)

T1-T2a, N1-3;

T2b-T4a, Any N

See Treatment of Primary and Neck (NASO-2)

Any T, Any N, M1

See Treatment of Primary and Neck (NASO-2)

Note: All recommendations are category $2 \mathrm{~A}$ unless otherwise indicated.

Clinical Trials: NCCN believes that the best management of any cancer patient is in a clinical trial. Participation in clinical trials is especially encouraged. 


\section{NCCN ${ }^{\oplus}$ Practice Guidelines in Oncology - v.2.2008

\section{CLINICAL STAGING TREATMENT OF PRIMARY AND NECK}

FOLLOW-UP

T1, NO, MO and T2a, NO, MO

T1-T2a, N1-3; T2b-T4a, any $N$

Cisplatin, $100 \mathrm{mg} / \mathrm{m}^{2}$
on days 1, 22, 43,
$\mathrm{RT}(\geq 70 \mathrm{~Gy}$ ) to
primary and gross
nodal disease
(category 1) and
bilateral neck:
$\geq 50$ Gy $|\rightarrow|$
Definitive $\mathrm{RT}^{\mathrm{a}}$ to nasopharynx and elective RT to neck

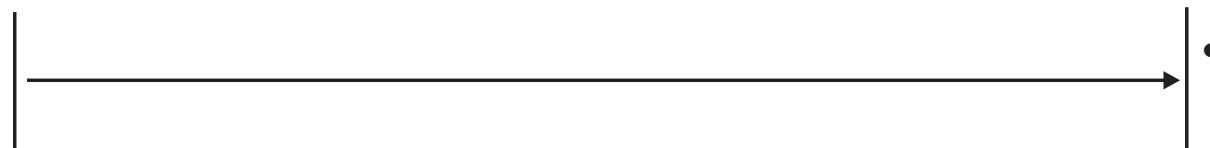
CI $x 4$ days; repeat every 4 wk $\times 3$

courses day $1+5-F U, 1,000$ $\mathrm{mg} / \mathrm{m}^{2}$
Cisplatin, $80 \mathrm{mg} / \mathrm{m}^{2}$

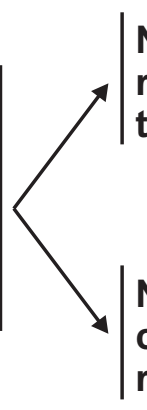

Neck: residual $\longrightarrow$ Neck tumor dissection

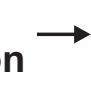

$\rightarrow$

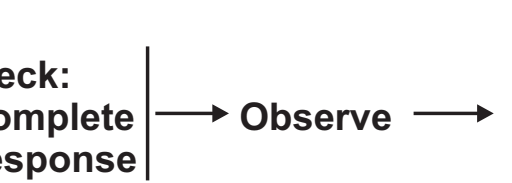

Any $\mathrm{T}$, any $\mathrm{N}, \mathrm{M} 1 \longrightarrow$

Platinum-based combination chemotherapy

If complete response
Definitive RT ${ }^{\mathrm{a}}$

to primary

and neck
- Physical exam:

- Year 1, every 1-3 mo

- Year 2, every 2-4 mo

- Year 3-5, every 4-6 mo

> $>5$ years, 6-12 mo

- Post-treatment baseline imaging of primary and neck recommended

- Reimaging as indicated by signs/symptoms on physical examination

- Chest imaging as clinically indicated

- TSH every 6-12 mo, if neck irradiated

- Speech, hearing and swallowing evaluation and rehabilitation as indicated - Smoking cessation counseling - Dental evaluation as indicated

Note: All recommendations are category $2 \mathrm{~A}$ unless otherwise indicated.

Clinical Trials: NCCN believes that the best management of any cancer patient is in a clinical trial. Participation in clinical trials is especially encouraged. 


\section{PRINCIPLES OF RADIATION THERAPY}

Definitive RT

- Primary and gross adenopathy:

$\geq 70$ Gy (2.0 Gy/day)

- Neck

- Uninvolved nodal stations:

$\geq 50$ Gy (2.0 Gy/day)

Radiation Techniques

Target delineation and optimal dose distribution require experience in head and neck imaging, a thorough understanding of patterns of disease spread, and experience in IMRT techniques. Standards for target definition, dose specification, fractionation (with and without concurrent chemotherapy), and normal tissue constraints are still evolving. IMRT, 3D and 2D conformal techniques may be used as appropriate depending on the stage, tumor location, physician training/experience and available physics support. 


\section{$\mathrm{NCCN}$

Newly diagnosed

Unresectable

(M0);

T4b, $\mathrm{N}$ any, or unresectable $\mathrm{N}+$

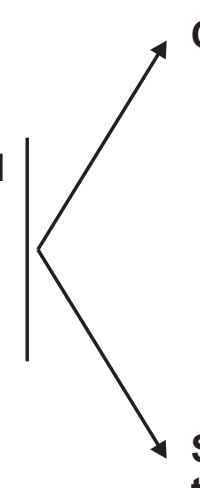

\section{Clinical trial preferred}
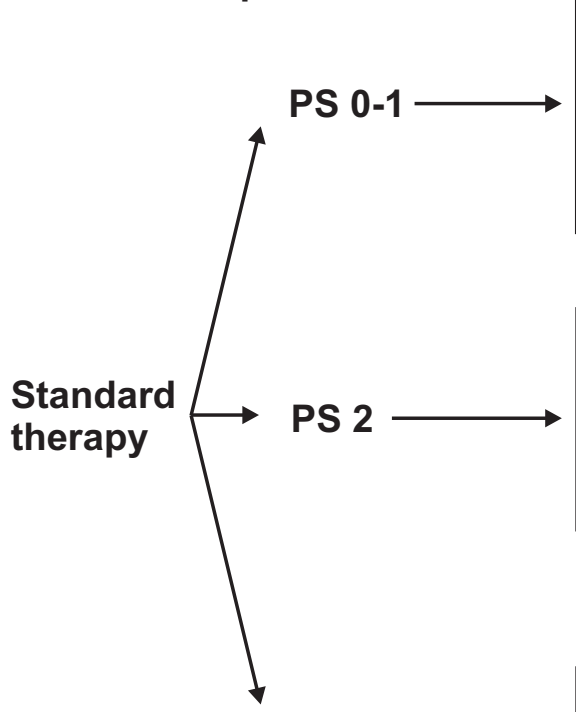

PS 3
Concurrent cisplatin or carboplatinbased chemotherapy ${ }^{a, b}+R^{c}$ (category 1)

or

Induction chemotherapy ${ }^{d}$ followed

by chemoradiation (category 3 )

Definitive $R T^{\mathrm{c}} \pm$ concurrent systemic therapy

or

Induction chemotherapy ${ }^{d}$ followed by RT (category 3)

$\longrightarrow \begin{aligned} & \text { Residual neck disease: } \\ & \text { Neck dissection, if } \\ & \text { feasible + primary site } \\ & \text { controlled }\end{aligned}$

Definitive RT ${ }^{\mathrm{C}}$

or

Best supportive care

a The single-agent cisplatin or carboplatin-based chemoradiotherapy regimens have not been compared in randomized trials. Therefore, no optimal standard regimen is defined. Combination chemotherapy regimens are more toxic and have not been directly compared to single-agent regimens.

b See Principles of Systemic Therapy (CHEM-A).

'See Principles of Radiation Therapy (ADV-A).

d Cisplatin $100 \mathrm{mg} / \mathrm{m}^{2}$ day $1+5-\mathrm{FU} 1000 \mathrm{mg} / \mathrm{m}^{2} / 24 \mathrm{hrs}$ continuous IV infusion for 120 hours for 3 cycles.

Note: All recommendations are category $2 \mathrm{~A}$ unless otherwise indicated.

Clinical Trials: NCCN believes that the best management of any cancer patient is in a clinical trial. Participation in clinical trials is especially encouraged. 


\section{$\mathrm{NCCN}^{\oplus}$ Practice Guidelines

\section{DIAGNOSIS}

TREATMENT OF HEAD AND NECK CANCER

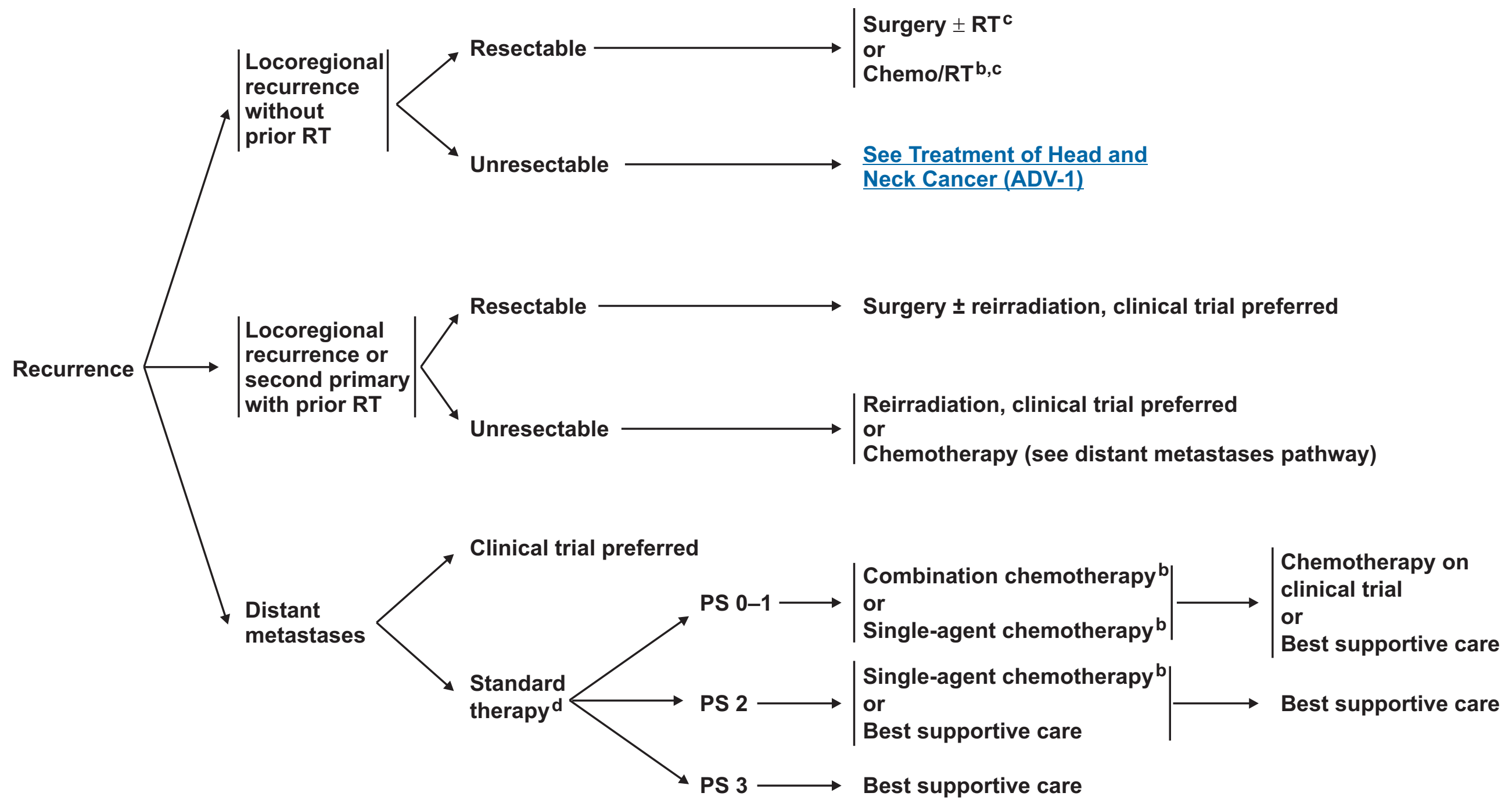

b See Principles of Systemic Therapy (CHEM-A).

c See Principles of Radiation Therapy (ADV-A).

Note: All recommendations are category $2 \mathrm{~A}$ unless otherwise indicated.

Clinical Trials: NCCN believes that the best management of any cancer patient is in a clinical trial. Participation in clinical trials is especially encouraged. 


\section{PRINCIPLES OF RADIATION THERAPY}

Concurrent chemoradiation (preferred)

Conventional fractionation:

- Primary and gross adenopathy

$\geq 70$ Gy (2.0 Gy/day)

- Neck

Uninvolved nodal stations:

44-50 Gy (2.0 Gy/day)

Altered fractionation (hyperfractionation or concomitant boost) regimens.

Examples

- Hyperfractionation:

81.6 Gy/7 wks (1.2 Gy/fraction BID)

- Modified fractionation

total dose $>70$ Gy and treatment course $<7$ wks

- IMRT 6 fraction/week

- Concomitant boost accelerated RT:

$72 \mathrm{~Gy} / 6$ wks (1.8 Gy/fraction, large field; $1.5 \mathrm{~Gy}$ boost as second daily fraction during last 12 treatment days)

- Conventional fractionation:

- Primary and gross adenopathy: $\geq 70$ Gy (2.0 Gy/day)

- Neck

Uninvolved nodal stations:

$\geq 50$ Gy (2.0 Gy/day)

\section{Radiation Techniques}

Target delineation and optimal dose distribution require experience in head and neck imaging, a thorough understanding of patterns of disease spread, and experience in IMRT techniques. Standards for target definition, dose specification, fractionation (with and without concurrent chemotherapy), and normal tissue constraints are still evolving. IMRT, 3D and 2D conformal techniques may be used as appropriate depending on the stage, tumor location, physician training/experience and available physics support.

\section{Chemoradiation}

Based on published data, concurrent chemoradiation typically uses conventional fractionation at $2.0 \mathrm{~g}$ per fraction to $\geq 70 \mathrm{~Gy}$ in 7 wks with single agent cisplatin given every 3 wks at $100 \mathrm{mg} / \mathrm{m}^{2} \times 3$ doses. Use of other fraction sizes (eg, $1.8 \mathrm{~Gy}$, conventional), multiagent chemotherapy or altered fractionation with chemotherapy has been evaluated with no consensus on the optimal approach. In general, the use of concurrent chemoradiation carries a high toxicity burden--altered fractionation or multiagent chemotherapy will likely further increase toxicity burden. For any chemoradiation approach, close attention should be paid to published reports for the specific chemotherapy agent, dose and schedule of administration.

Chemoradiation should be performed by an experienced team and include substantial supportive care.

Note: All recommendations are category $2 \mathrm{~A}$ unless otherwise indicated.

Clinical Trials: NCCN believes that the best management of any cancer patient is in a clinical trial. Participation in clinical trials is especially encouraged. 


\section{PRINCIPLES OF SYSTEMIC THERAPY (Page 1 of 2 )}

The choice of chemotherapy should be individualized based on patient characteristics (performance status, goals of therapy).

\section{Squamous Cell Cancers}

Maxillary Sinus, Ethmoid Sinus, Lip, Oral Cavity, Oropharynx, Hypopharynx, Glottic larynx, Supraglottic larynx, Occult Primary

Primary Systemic Therapy + concurrent RT

- Cisplatin alone ${ }^{1,2}$ (preferred) $)^{3,4}$

- 5-FU/hydroxyurea ${ }^{5}$

- Cisplatin/paclitaxel ${ }^{5}$

- Cisplatin/infusional 5-FU 5

- Carboplatin/infusional 5-FU 6

- Cetuximab 7

Postoperative Chemoradiation

- Cisplatin alone $8,9,10$

Induction chemotherapy

- Docetaxel/cisplatin/5-FU11,12,13

\section{Nasopharynx}

Chemoradiation followed by adjuvant chemotherapy

- Cisplatin + RT followed by Cisplatin/5-FU ${ }^{14}$

\begin{tabular}{|c|c|c|}
\hline \multicolumn{3}{|c|}{ Unresectable/Recurrent Head and Neck Cancers } \\
\hline $\begin{array}{l}\text { Combination therapy } \\
\text { - Cisplatin or carboplatin } \\
+5-\text { FU }^{15,16} \pm \\
\text { cetuximab }^{17} \\
\text { - Cisplatin or carboplatin } \\
+ \text { docetaxel or } \\
\text { paclitaxel }^{6} \\
\text { - Cisplatin/cetuximab } \\
\end{array}$ & $\begin{array}{l}\text { Single agent } \\
\text { - Cisplatin } \\
\text { - Carboplatin } \\
\text { - Paclitaxel } \\
\text { - Docetaxel } \\
\text { - 5-FU } \\
\text { - Methotrexate }\end{array}$ & $\begin{array}{l}\text { Ifosfamide } \\
\text { Bleomycin } \\
\text { Gemcitabine } 19 \\
\text { (nasopharyngeal) } \\
\text { - Cetuximab }\end{array}$ \\
\hline
\end{tabular}

See References on page CHEM-A 2 of 2

Note: All recommendations are category $2 \mathrm{~A}$ unless otherwise indicated.

Clinical Trials: NCCN believes that the best management of any cancer patient is in a clinical trial. Participation in clinical trials is especially encouraged. 


\section{PRINCIPLES OF SYSTEMIC THERAPY (Page 2 of 2) REFERENCES}

${ }^{1}$ Forastiere AA, Goepfert H, Maor M, et al. Concurrent chemotherapy and radiotherapy for organ preservation in advanced laryngeal cancer. N Engl J Med 2003;349:2091-8

${ }^{2}$ Adelstein DJ, Li Y, Adams GL, et al. An intergroup phase III comparison of standard radiation therapy and two schedules of concurrent chemoradiotherapy in patients with unresectable squamous cell head and neck cancer. J Clin Oncol 2003;21(1):92-98.

${ }^{3}$ Bachaud JM, Cohen-Jonathan E, Alzieu C, et al. Combined postoperative radiotherapy and weekly cisplatin infusion for locally advanced head and neck carcinoma: final report of a randomized trial. Int J Radiat Oncol Biol Phys 1996;36(5):999-1004. (cisplatin 50 mg IV weekly)

${ }^{4}$ Jeremic B, Milicic B, Dagovic A, et al. Radiation therapy with or without concurrent low-dose daily chemotherapy in locally advanced, nonmetastatic squamous cell carcinoma of the head and neck. Clin Oncol 2004;22(17):35403548. (cisplatin $6 \mathrm{mg} / \mathrm{m} 2$ daily)

${ }^{5}$ Garden AS, Harris J, Vokes EE, et al. Preliminary results of Radiation Therapy Oncology Group 97-03: A randomized phase II trial of concurrent radiation and chemotherapy for advanced squamous cell carcinomas of the head and neck. $\mathrm{J}$ Clin Oncol 2004;22:2856-2864.

${ }^{6}$ Denis F, Garaud P, Bardet E, et al. Final results of the 94-01 French Head and Neck Oncology and Radiotherapy Group randomized trial comparing radiotherapy alone with concomitant radiochemotherapy in advanced-stage oropharynx carcinoma. J Clin Oncol 2004;22(1):69-76.

${ }^{7}$ Bonner JA, Harari PM, Giralt J, et al. Radiotherapy plus cetuximab for squamous cell carcinoma of the head and neck. N Engl J Med 2006;354:56778.

${ }^{8}$ Cooper JS, Pajak TF, Forastiere AA, et al. Postoperative concurrent radiotherapy and chemotherapy for high-risk squamous-cell carcinoma of the head and neck. N Engl J Med 2004;350:1937-44

${ }^{9}$ Bernier J, Domenge C, Ozsahin M, et al. Postoperative irradiation with or without concomitant chemotherapy for locally advanced head and neck cancer. N Engl J Med 2004;350:1945-52.

${ }^{10}$ Bernier J, Cooper JS, Pajak TF, et al. Defining risk levels in locally advanced head and neck cancers: A comparative analysis of concurrent postoperative radiation plus chemotherapy trials of the EORTC (\#22931) and RTOG (\# 9501). Head Neck 2005;27:843-850.
${ }^{11}$ Schrijvers D, Van Herpen C, Kerger J, et al. Docetaxel, cisplatin and 5fluorouracil in patients with locally advanced unresectable head and neck cancer: a phase I-II feasibility study. Annals of Oncology 2004;15:638-645.

${ }^{12}$ Vermorken JB, Remenar E, van Herpen C, et al; EORTC 24971/TAX 323 Study Group. Cisplatin, fluorouracil, and docetaxel in unresectable head and neck cancer. N Engl J Med 2007;357(17):1695-1704.

${ }^{13}$ Posner MR, Hershock DM, Blajman CR, et al. Cisplatin and fluorouracil alone or with docetaxel in head and neck cancer. N Engl J Med 2007;357(17):17051715.

${ }^{14} \mathrm{Al}$-Sarraf M, LeBlanc M, Giri PG, et al. Chemoradiotherapy versus radiotherapy in patients with advanced nasopharyngeal cancer: phase III randomized Intergroup study 0099. J Clin Oncol 1998;16:1310-1317.

15 Gibson MK, Li Y, Murphy B, et al. Randomized phase III evaluation of cisplatin plus fluorouracil versus cisplatin plus paclitaxel in advanced head and neck cancer (E1395): An Intergroup Trial of the Eastern Cooperative Oncology Group. J Clin Oncol 2005;23(15):3562-3567.

${ }^{16}$ Forastiere AA, Metch B, Schuller DE, et al. Randomized comparison of cisplatin plus flurouracil and carboplatin plus fluorouracil versus methotrexate in advanced squamous cell carcinoma of the head and neck: A Southwest Oncology Group Study. J Clin Oncol 1992;10(8):1245-1251.

17 Vermorken J, Mesia R, Vega V, et al. Cetuximab extends survival of patients with recurrent or metastatic SCCHN when added to first line platinum based therapy - Results of a randomized phase III (Extreme) study [abstract]. ASCO Annual Meeting Proceedings. J Clin Oncol 2007;25:6091.

${ }^{18}$ Burtness B, Goldwasser MA, Flood W, et al. Phase III randomized trial of cisplatin plus placebo versus cisplatin plus cetuximab in metastatic/recurrent head and neck cancer: An Eastern Cooperative Oncology Group Study. J Clin Oncol 2005;23:8646-8654.

${ }^{19}$ Zhang L, Zhang Y, Huang PY, et al. Phase II clinical study of gemcitabine in the treatment of patients with advanced nasopharyngeal carcinoma after the failure of platinum-based chemotherapy. Cancer Chemother Pharmacol 2008;61(1):33-38. Epub 2007 Mar 20.

${ }^{20}$ Vermorken JB, Trigo J, Hitt R, et al. Open-label, uncontrolled, multicenter phase II study to evaluate the efficacy and toxicity of cetuximab as a single agent in patients with recurrent and/or metastatic squamous cell carcinoma of the head and neck who failed to respond to platinum-based therapy. J Clin Oncol 2007;25(16):2171-2177.

Note: All recommendations are category $2 \mathrm{~A}$ unless otherwise indicated.

Clinical Trials: NCCN believes that the best management of any cancer patient is in a clinical trial. Participation in clinical trials is especially encouraged. 


\section{Staging}

\section{Table 1: 2002 American Joint Committee on Cancer (AJCC)} TNM Staging System for the Lip and Oral Cavity

(Nonepithelial tumors are not included, such as those of lymphoid tissue, soft tissue, bone, and cartilage)

\section{Primary Tumor $(\mathrm{T})$}

TX Primary tumor cannot be assessed

T0 No evidence of primary tumor

Tis Carcinoma in situ

T1 Tumor $2 \mathrm{~cm}$ or less in greatest dimension

T2 Tumor more than $2 \mathrm{~cm}$ but not more than $4 \mathrm{~cm}$ in greatest dimension

T3 Tumor more than $4 \mathrm{~cm}$ in greatest dimension

T4 (lip) Tumor invades through cortical bone, inferior alveolar nerve, floor of mouth, or skin of face (ie, chin or nose)

T4a (oral cavity) Tumor invades adjacent structures (eg, through cortical bone, into deep [extrinsic] muscle of tongue [genioglossus, hyoglossus, palatoglossus, and styloglossus], maxillary sinus, skin of face)

T4b Tumor invades masticator space, pterygoid plates, or skull base and/or encases internal carotid artery

*Note: Superficial erosion alone of bone/tooth socket by gingival primary is not sufficient to classify as T4.

\section{Regional Lymph Nodes (N)}

NX Regional nodes cannot be assessed

No No regional lymph node metastasis

N1 Metastasis in a single ipsilateral lymph node, $3 \mathrm{~cm}$ or less in greatest dimension

N2

Metastasis in a single ipsilateral lymph node, more than $3 \mathrm{~cm}$ but not more than $6 \mathrm{~cm}$ in greatest dimension; or in multiple ipsilateral lymph nodes, none more than $6 \mathrm{~cm}$ in greatest dimension; or in bilateral or contralateral lymph nodes, none more than $6 \mathrm{~cm}$ in greatest dimension

N2a Metastasis in single ipsilateral lymph node more than 3 $\mathrm{cm}$ but not more than $6 \mathrm{~cm}$ in greatest dimension
N2b Metastasis in multiple ipsilateral lymph nodes, none more than $6 \mathrm{~cm}$ in greatest dimension

N2c Metastasis in bilateral or contralateral lymph nodes, none more than $6 \mathrm{~cm}$ in greatest dimension

Metastasis in a lymph node more than $6 \mathrm{~cm}$ in greatest dimension

\section{Distant Metastasis (M) \\ MX Distant metastasis cannot be assessed \\ MO No distant metastasis \\ M1 Distant metastasis}

\begin{tabular}{llll}
\multicolumn{3}{l}{ Stage Grouping } & \\
Stage $\mathbf{0}$ & Tis & N0 & M0 \\
Stage I & T1 & N0 & M0 \\
Stage II & T2 & N0 & M0 \\
Stage III & T3 & N0 & M0 \\
& T1 & N1 & M0 \\
& T2 & N1 & M0 \\
& T3 & N1 & M0 \\
Stage IVA & T4a & N0 & M0 \\
& T4a & N1 & M0 \\
& T1 & N2 & M0 \\
& T2 & N2 & M0 \\
& T3 & N2 & M0 \\
& T4a & N2 & M0 \\
Stage IVB & Any T & N3 & M0 \\
& T4b & Any N & M0 \\
Stage IVC & Any T & Any N & M1
\end{tabular}

Histologic Grade (G)

GX Grade cannot be assessed

G1 Well differentiated

G2 Moderately differentiated

G3 Poorly differentiated

Used with the permission of the American Joint Committee on Cancer (AJCC), Chicago, Illinois. The original and primary source for this information is the AJCC Cancer Staging Manual, Sixth Edition (2002) published by Springer-Verlag New York. (For more information, visit www.cancerstaging.net.) Any citation or quotation of this material must be credited to the AJCC as its primary source. The inclusion of this information herein does not authorize any reuse or further distribution without the expressed, written permission of Springer-Verlag New York, Inc., on behalf of the AJCC. 


\section{Table 2: 2002 American Joint Committee on Cancer (AJCC) TNM Staging System for the Pharynx (Including Base of} Tongue, Soft Palate, and Uvula)

(Nonepithelial tumors are not included, such as those of lymphoid tissue, soft tissue, bone, and cartilage)

\section{Primary Tumor (T)}

TX Primary tumor cannot be assessed

T0 No evidence of primary tumor

Tis Carcinoma in situ

\section{Nasopharynx}

T1 Tumor confined to the nasopharynx

T2 Tumor extends to soft tissues

T2a Tumor extends to the oropharynx and/or nasal cavity without parapharyngeal extension*

T2b Any tumor with parapharyngeal extension*

T3 Tumor invades bony structures and/or paranasal sinuses

T4 Tumor with intracranial extension and/or involvement of cranial nerves, infratemporal fossa, hypopharynx, orbit, or masticator space

*Note: Parapharyngeal extension denotes posterolateral infiltration of tumor beyond the pharyngobasilar fascia.

\section{Oropharynx}

T1 Tumor $2 \mathrm{~cm}$ or less in greatest dimension

T2 Tumor more than $2 \mathrm{~cm}$ but not more than $4 \mathrm{~cm}$ in greatest dimension

T3 Tumor more than $4 \mathrm{~cm}$ in greatest dimension

T4a Tumor invades the larynx, deep/extrinsic muscle of tongue, medial pterygoid, hard palate, or mandible

T4b Tumor invades lateral pterygoid muscle, pterygoid plates, lateral nasopharynx, or skull base or encases carotid artery

\section{Hypopharynx}

T1 Tumor limited to one subsite of hypopharynx and $2 \mathrm{~cm}$ or less in greatest dimension

T2

Tumor invades more than one subsite of hypopharynx or an adjacent site, or measures more than $2 \mathrm{~cm}$ but not more than $4 \mathrm{~cm}$ in greatest diameter without fixation of hemilarynx

\section{T3}

T4a

T4b

Tumor more than $4 \mathrm{~cm}$ in greatest dimension or with fixation of hemilarynx

Tumor invades thyroid/cricoid cartilage, hyoid bone, thyroid gland, esophagus, or central compartment soft tissue $^{*}$

T4

Tumor invades prevertebral fascia, encases carotid artery, or involves mediastinal structures

*Note: Central compartment soft tissue includes prelaryngeal strap muscles and subcutaneous fat.

\section{Regional Lymph Nodes (N)}

\section{Nasopharynx}

The distribution and the prognostic impact of regional lymph node spread from nasopharynx cancer, particularly of the undifferentiated type, are different from those of other head and neck mucosal cancers and justify the use of a different $\mathrm{N}$ classification system.

NX

No

N1

N2

N3

N3a

N3b Extension to the supraclavicular fossa**

*Note: Midline nodes are considered ipsilateral nodes.

${ }^{* *}$ Supraclavicular zone or fossa is relevant to the staging of

nasopharyngeal carcinoma and is the triangular region originally described by Ho. It is defined by three points: (1) the superior margin of the sternal end of the clavicle; (2) the superior margin of the lateral end of the clavicle, and (3) the point where the neck meets the shoulder. Note that this would include caudal portions of levels IV and V. All cases with lymph nodes (whole or part) in the fossa are considered N3b.

\section{Continued..}




\section{Table 2 - Continued}

\section{Oropharynx and Hypopharynx}

NX Regional lymph nodes cannot be assessed

No No regional lymph node metastasis

N1 Metastasis in a single ipsilateral lymph node, $3 \mathrm{~cm}$ or less in greatest dimension

N2 Metastasis in a single ipsilateral lymph node, more than 3 $\mathrm{cm}$ but not more than $6 \mathrm{~cm}$ in greatest dimension, or in multiple ipsilateral lymph nodes, none more than $6 \mathrm{~cm}$ in greatest dimension, or in bilateral or contralateral lymph nodes, none more than $6 \mathrm{~cm}$ in greatest dimension

N2a Metastasis in a single ipsilateral lymph node more than 3 $\mathrm{cm}$ but not more than $6 \mathrm{~cm}$ in greatest dimension

N2b Metastasis in multiple ipsilateral lymph nodes, none more than $6 \mathrm{~cm}$ in greatest dimension

N2c Metastasis in bilateral or contralateral lymph nodes, none more than $6 \mathrm{~cm}$ in greatest dimension

N3

Metastasis in a lymph node more than $6 \mathrm{~cm}$ in greatest dimension

\section{Distant Metastasis (M)}

MX Distant metastasis cannot be assessed

Mo No distant metastasis

M1 Distant metastasis

\begin{tabular}{lccc}
\multicolumn{4}{c}{ Stage Grouping: } \\
Stage $\mathbf{0}$ & Tis & N0 & M0 \\
Stage I & T1 & N0 & M0 \\
Stage IIA & T2a & N0 & M0 \\
Stage IIB & T1 & N1 & M0 \\
& T2 & N1 & M0 \\
& T2a & N1 & M0 \\
& T2b & N0 & M0 \\
& T2b & N1 & M0 \\
Stage III & T1 & N2 & M0 \\
& T2a & N2 & M0 \\
& T2b & N2 & M0
\end{tabular}

$\begin{array}{cccc} & \text { T3 } & \text { N0 } & \text { M0 } \\ & \text { T3 } & \text { N1 } & \text { M0 } \\ \text { Stage IVA } & \text { T3 } & \text { N2 } & \text { M0 } \\ & \text { T4 } & \text { N0 } & \text { M0 } \\ & \text { T4 } & \text { N2 } & \text { M0 } \\ \text { Stage IVB } & \text { Any T } & \text { N3 } & \text { M0 } \\ \text { Stage IVC } & \text { Any T } & \text { Any N } & \text { M1 }\end{array}$

Stage Grouping: Oropharynx, Hypopharynx

Stage 0 Tis NO MO

Stage I $\quad \mathrm{T} 1 \quad \mathrm{~N} 0 \mathrm{M}$

$\begin{array}{llll}\text { Stage II } & \text { T2 } & \text { N0 } & \text { MO }\end{array}$

$\begin{array}{llll}\text { Stage III T3 NO } & \text { MO }\end{array}$

$\begin{array}{lll}\mathrm{T} 1 & \mathrm{~N} 1 & \mathrm{M} 0 \\ \mathrm{~T} 2 & \mathrm{~N} 1 & \mathrm{M} 0\end{array}$

T3 N1 M0

Stage IVA T4a N0 M0

T4a N1 M0

T1 N2 M0

T2 N2 M0

T3 N2 M0

T4a N2 M0

Stage IVB T4b Any N MO

Any $\mathrm{T} \quad \mathrm{N} 3 \quad \mathrm{M} 0$

Stage IVC Any T Any N M1

Histologic Grade (G)

- Oropharynx

- Hypopharynx

GX Grade cannot be assessed

G1 Well differentiated

G2 Moderately differentiated

G3 Poorly differentiated

Used with the permission of the American Joint Committee on Cancer (AJCC), Chicago, Illinois. The original and primary source for this information is the AJCC Cancer Staging Manual, Sixth Edition (2002) published by Springer-Verlag New York. (For more information, visit www.cancerstaging. net.) Any citation or quotation of this material must be credited to the AJCC as its primary source. The inclusion of this information herein does not authorize any reuse or further distribution without the expressed, written permission of Springer-Verlag New York, Inc., on behalf of the AJCC. 
Table 3: 2002 American Joint Committee on Cancer (AJCC) TNM Staging System for the Larynx

(Nonepithelial tumors are not included, such as those of lymphoid tissue, soft tissue, bone, and cartilage)

\section{Primary Tumor $(\mathrm{T})$}

TX Primary tumor cannot be assessed

T0 No evidence of primary tumor

Tis Carcinoma in situ

\section{Supraglottis}

T1 Tumor limited to one subsite of supraglottis with normal vocal cord mobility

T2 Tumor invades mucosa of more than one adjacent subsite of supraglottis or glottis or region outside the supraglottis (eg, mucosa of base of tongue, vallecula, medial wall of pyriform sinus) without fixation of the larynx

T3 Tumor limited to larynx with vocal cord fixation and/or invades any of the following: postcricoid area, preepiglottic tissues, paraglottic space, and/or minor thyroid cartilage erosion (eg, inner cortex)

T4a Tumor invades through the thyroid cartilage and/or invades tissues beyond the larynx (eg, trachea, soft tissues of neck including deep extrinsic muscle of the tongue, strap muscles, thyroid, or esophagus)

T4b Tumor invades prevertebral space, encases carotid artery, or invades mediastinal structures

\section{Glottis}

T1

Tumor limited to the vocal cord(s) (may involve anterior or posterior commissure) with normal mobility

T1a Tumor limited to one vocal cord

T1b Tumor involves both vocal cords

T2 Tumor extends to supraglottis and/or subglottis, and/or with impaired vocal cord mobility

T3 Tumor limited to the larynx with vocal cord fixation and/or invades paraglottic space, and/or minor thyroid cartilage erosion (eg, inner cortex)

T4a Tumor invades through the thyroid cartilage and/or invades tissues beyond the larynx (eg, trachea, soft tissues of neck including deep extrinsic muscle of the tongue, strap muscles, thyroid, or esophagus)

T4b Tumor invades prevertebral space, encases carotid artery, or invades mediastinal structures

\section{Subglottis}

T1 Tumor limited to the subglottis

T2 Tumor extends to vocal cord(s) with normal or impaired mobility

T3 Tumor limited to larynx with vocal cord fixation

T4a Tumor invades cricoid or thyroid cartilage and/or invades tissues beyond the larynx (eg, trachea, soft tissues of neck including deep extrinsic muscle of the tongue, strap muscles, thyroid, or esophagus)

T4b Tumor invades prevertebral space, encases carotid artery, or invades mediastinal structures

\section{Regional Lymph Nodes (N)}

NX Regional lymph nodes cannot be assessed

No No regional lymph node metastasis

N1 Metastasis in a single ipsilateral lymph node, $3 \mathrm{~cm}$ or less in greatest dimension

N2 Metastasis in a single ipsilateral lymph node, more than 3 $\mathrm{cm}$ but not more than $6 \mathrm{~cm}$ in greatest dimension; or in multiple ipsilateral lymph nodes, none more than $6 \mathrm{~cm}$ in greatest dimension; or in bilateral or contralateral lymph nodes, none more than $6 \mathrm{~cm}$ in greatest dimension

N2a Metastasis in single ipsilateral lymph node, more than 3 $\mathrm{cm}$ but not more than $6 \mathrm{~cm}$ in greatest dimension

N2b Metastasis in multiple ipsilateral lymph nodes, none more than $6 \mathrm{~cm}$ in greatest dimension

N2c Metastasis in bilateral or contralateral lymph nodes, none more than $6 \mathrm{~cm}$ in greatest dimension

N3 Metastasis in a lymph node, more than $6 \mathrm{~cm}$ in greatest dimension

\section{Distant Metastasis (M)}

MX Distant metastasis cannot be assessed

MO No distant metastasis

M1 Distant metastasis

Continued... 


\section{Table 3 - Continued}

\section{Stage Grouping}

\begin{tabular}{|c|c|c|c|c|}
\hline Stage 0 & Tis & No & M0 & Histologic Grade (G) \\
\hline Stage I & T1 & No & M0 & GX Grade cannot be \\
\hline Stage II & T2 & No & MO & assessed \\
\hline \multirow[t]{4}{*}{ Stage III } & T3 & No & MO & G1 Well differentiated \\
\hline & T1 & N1 & MO & G2 Moderately \\
\hline & T2 & N1 & MO & differentiated \\
\hline & T3 & N1 & MO & G3 Poorly differentiated \\
\hline \multirow[t]{6}{*}{ Stage IVA } & $\mathrm{T} 4 \mathrm{a}$ & No & MO & \\
\hline & $\mathrm{T} 4 \mathrm{a}$ & N1 & MO & \\
\hline & T1 & N2 & M0 & \\
\hline & T2 & N2 & MO & \\
\hline & T3 & N2 & M0 & \\
\hline & $\mathrm{T} 4 \mathrm{a}$ & N2 & M0 & \\
\hline \multirow[t]{2}{*}{ Stage IVB } & $\mathrm{T} 4 \mathrm{~b}$ & Any $\mathrm{N}$ & M0 & \\
\hline & Any $T$ & N3 & M0 & \\
\hline Stage IVC & Any $\mathrm{T}$ & Any $\mathrm{N}$ & M1 & \\
\hline
\end{tabular}

Used with the permission of the American Joint Committee on Cancer (AJCC), Chicago, Illinois. The original and primary source for this information is the AJCC Cancer Staging Manual, Sixth Edition (2002) published by Springer-Verlag New York. (For more information, visit www.cancerstaging.net.) Any citation or quotation of this material must be credited to the AJCC as its primary source. The inclusion of this information herein does not authorize any reuse or further distribution without the expressed, written permission of Springer-Verlag New York, Inc., on behalf of the AJCC. 


\section{Table 4}

2002 American Joint Committee on Cancer (AJCC)

TNM Staging System for the Major Salivary Glands (Parotid,

Submandibular, and Sublingual)

\section{Primary Tumor ( $T$ )}

TX Primary tumor cannot be assessed

T0 No evidence of primary tumor

T1 Tumor $2 \mathrm{~cm}$ or less in greatest dimension without extraparenchymal extension*

T2 Tumor more than $2 \mathrm{~cm}$ but not more than $4 \mathrm{~cm}$ in greatest dimension without extraparenchymal extension*

T3

Tumor more than $4 \mathrm{~cm}$ and/or tumor having

extraparenchymal extension*

T4a Tumor invades skin, mandible, ear canal, and/or facial nerve

T4b Tumor invades skull base and/or pterygoid plates and/or encases carotid artery

*Note: Extraparenchymal extension is clinical or macroscopic evidence of invasion of soft tissues. Microscopic evidence alone does not constitute extraparenchymal extension for classification purposes.

\section{Regional Lymph Nodes (N)}

NX Regional lymph nodes cannot be assessed

No No regional lymph node metastasis

N1 Metastasis in a single ipsilateral lymph node, $3 \mathrm{~cm}$ or less in greatest dimension

N2 Metastasis in a single ipsilateral lymph node, more than 3 $\mathrm{cm}$ but not more than $6 \mathrm{~cm}$ in greatest dimension; or in multiple ipsilateral lymph nodes, none more than $6 \mathrm{~cm}$ in greatest dimension; or in bilateral or contralateral lymph nodes, none more than $6 \mathrm{~cm}$ in greatest dimension

N2a Metastasis in a single ipsilateral lymph node, more than 3 $\mathrm{cm}$ but not more than $6 \mathrm{~cm}$ in greatest dimension

N2b Metastasis in multiple ipsilateral lymph nodes, none more than $6 \mathrm{~cm}$ in greatest dimension
N2c Metastasis in bilateral or contralateral lymph nodes, none more than $6 \mathrm{~cm}$ in greatest dimension

Metastasis in a lymph node, more than $6 \mathrm{~cm}$ in greatest dimension

\section{Distant Metastasis (M)}

Mx Distant metastasis cannot be assessed

Mo No distant metastasis

M1 Distant metastasis

\section{Stage Grouping}

$\begin{array}{lccc}\text { Stage I } & \text { T1 } & \text { N0 } & \text { M0 } \\ \text { Stage II } & \text { T2 } & \text { N0 } & \text { M0 } \\ \text { Stage III } & \text { T3 } & \text { N0 } & \text { M0 } \\ & \text { T1 } & \text { N1 } & \text { M0 } \\ & \text { T2 } & \text { N1 } & \text { M0 } \\ \text { Stage IVA } & \text { T3 } & \text { N1 } & \text { M0 } \\ & \text { T4a } & \text { N0 } & \text { M0 } \\ & \text { T1 } & \text { N2 } & \text { M0 } \\ & \text { T2 } & \text { N2 } & \text { M0 } \\ & \text { T3 } & \text { N2 } & \text { M0 } \\ \text { Stage IVB } & \text { T4a } & \text { N2 } & \text { M0 } \\ & \text { T4b } & \text { Any N } & \text { M0 } \\ \text { Stage IVC } & \text { Any T } & \text { N3 } & \text { M0 } \\ & \text { Any } & \text { Any N } & \text { M1 }\end{array}$

Used with the permission of the American Joint Committee on Cancer (AJCC), Chicago, Illinois. The original and primary source for this information is the AJCC Cancer Staging Manual, Sixth Edition (2002) published by Springer-Verlag New York. (For more information, visit www.cancerstaging.net.) Any citation or quotation of this material must be credited to the AJCC as its primary source. The inclusion of this information herein does not authorize any reuse or further distribution without the expressed, written permission of Springer-Verlag New York, Inc., on behalf of the AJCC. 


\section{Table 5: 2002 American Joint Committee on Cancer (AJCC) TNM Staging System for the Nasal Cavity and Paranasal}

\section{Sinuses}

(Nonepithelial tumors are not included, such as those of lymphoid tissue, soft tissue, bone, and cartilage)

\section{Primary Tumor $(T)$ \\ TX Primary tumor cannot be assessed \\ T0 No evidence of primary tumor \\ Tis Carcinoma in situ}

\section{Maxillary Sinus}

T1 Tumor limited to maxillary sinus mucosa with no erosion or destruction of bone

T2 Tumor causing bone erosion or destruction including extension into the hard palate and/or middle nasal meatus, except extension to posterior wall of maxillary sinus and pterygoid plates

T3 Tumor invades any of the following: bone of the posterior wall of maxillary sinus, subcutaneous tissues, floor or medial wall of orbit, pterygoid fossa, ethmoid sinuses

T4a Tumor invades anterior orbital contents, skin of cheek, pterygoid plates, infratemporal fossa, cribriform plate, sphenoid or frontal sinuses

T4b Tumor invades any of the following: orbital apex, dura, brain, middle cranial fossa, cranial nerves other than maxillary division of trigeminal nerve $\left(\mathrm{V}_{2}\right)$, nasopharynx, or clivus

\section{Nasal Cavity and Ethmoid Sinus}

T1 Tumor restricted to any one subsite, with or without bony invasion

\section{Tumor invading two subsites in a single region or} extending to involve an adjacent region within the nasoethmoidal complex, with or without bony invasion
T4a

T4b
Tumor invades any of the following: anterior orbital contents, skin of nose or cheek, minimal extension to anterior cranial fossa, pterygoid plates, sphenoid or frontal sinuses

Tumor invades any of the following: orbital apex, dura, brain, middle cranial fossa, cranial nerves other than $\left(V_{2}\right)$, nasopharynx, or clivus

\section{Regional Lymph Nodes (N)}

NX Regional lymph nodes cannot be assessed

No No regional lymph node metastasis

N1 Metastasis in a single ipsilateral lymph node, $3 \mathrm{~cm}$ or less in greatest dimension

N2 Metastasis in a single ipsilateral lymph node, more than 3 $\mathrm{cm}$ but not more than $6 \mathrm{~cm}$ in greatest dimension; or in multiple ipsilateral lymph nodes, none more than $6 \mathrm{~cm}$ in greatest dimension; or in bilateral or contralateral lymph nodes, none more than $6 \mathrm{~cm}$ in greatest dimension

N2a Metastasis in a single ipsilateral lymph node, more than 3 $\mathrm{cm}$ but not more than $6 \mathrm{~cm}$ in greatest dimension

N2b Metastasis in multiple ipsilateral lymph nodes, none more than $6 \mathrm{~cm}$ in greatest dimension

N2c Metastasis in bilateral or contralateral lymph nodes, none more than $6 \mathrm{~cm}$ in greatest dimension

N3 Metastasis in a lymph node, more than $6 \mathrm{~cm}$ in greatest dimension

\section{Distant Metastasis (M)}

MX Distant metastasis cannot be assessed

Mo No distant metastasis

M1 Distant metastasis 
Table 5 - Continued

\section{Stage Grouping}

Stage $0 \quad$ Tis

Stage I $\quad T 1$

Stage II T2

Stage III T3

$\mathrm{T} 1$

T2

T3

Stage IVA T4a

T4a

T1

T2

T3

$\mathrm{T} 4 \mathrm{a}$
Histologic Grade (G)

GX Grade cannot be assessed

G1 Well differentiated

G2 Moderately

differentiated

G3 Poorly differentiated

$\begin{array}{cccc}\text { Stage IVB } & \text { T4b } & \text { Any N } & \text { M0 } \\ & \text { Any T } & \text { N3 } & \text { M0 } \\ \text { Stage IVC } & \text { Any T } & \text { Any N } & \text { M1 }\end{array}$

Used with the permission of the American Joint Committee on Cancer (AJCC), Chicago, Illinois. The original and primary source for this information is the AJCC Cancer Staging Manual, Sixth Edition (2002) published by Springer-Verlag New York. (For more information, visit www.cancerstaging. net.) Any citation or quotation of this material must be credited to the AJCC as its primary source. The inclusion of this information herein does not authorize any reuse or further distribution without the expressed, written permission of Springer-Verlag New York, Inc., on behalf of the AJCC. 


\section{Manuscript}

\section{NCCN Categories of Evidence and Consensus}

Category 1: Based on high-level evidence and uniform consensus.

Category 2A: Based on lower-level evidence including clinical experience and uniform consensus.

Category 2B: Based on lower-level evidence including clinical experience and nonuniform consensus (but no major disagreement).

Category 3: Based on any level of evidence but reflects major disagreement.

All recommendations are category $2 \mathrm{~A}$ unless otherwise noted.

\section{Overview}

The NCCN Head and Neck (H\&N) Cancers guidelines address tumors arising in the lip, oral cavity, oropharynx, hypopharynx, nasopharynx, glottic and supraglottic larynx, paranasal (ethmoid and maxillary) sinuses, and salivary glands; occult primary cancer is also addressed (see Figure 1). As background to the discussion of these guidelines, a brief overview of the epidemiology and management of H\&N cancer is provided.

\section{Incidence and Etiology}

Approximately 47,560 new cases of oral cavity, pharyngeal, and laryngeal cancers are estimated to occur in 2008. This accounts for about $3 \%$ of new cancer cases in the United States. An estimated 11,260 deaths from H\&N cancers will occur in 2008. ${ }^{1}$ Alcohol and tobacco abuse are common etiologic factors in cancers of the oral cavity, oropharynx, hypopharynx, and larynx. Moreover, because the entire aerodigestive tract epithelium may be exposed to these carcinogens, patients with $\mathrm{H} \& \mathrm{~N}$ cancer are at risk for developing second primary neoplasms of the H\&N, lung, and esophagus. Human papilloma virus (HPV) infection appears to be a risk factor for some squamous cancers of the oropharynx (predominately cancers of the lingual and palatine tonsils). ${ }^{2-7}$

\section{Staging}

Stage at diagnosis is the most predictive factor of survival. The TNM staging systems developed by the American Joint Committee on Cancer (AJCC) for the lip and oral cavity, pharynx (nasopharynx, oropharynx, and hypopharynx), larynx (glottis and supraglottis), major salivary glands (parotid, submandibular, and sublingual), and nasal cavity and paranasal sinuses are shown in Tables $1,2,3,4$, and $\underline{5}$, respectively. ${ }^{8}$ The 2002 AJCC staging classification was used as a basis for the NCCN's treatment recommendations for the pharynx (see Table 2). Definitions for regional lymph node $(\mathrm{N})$ involvement and spread to distant metastatic sites (M) are uniform except for $\mathrm{N}$ staging of nasopharyngeal carcinoma. Definitions for staging the primary tumor (T), based on its size, are uniform for the lip, oral cavity, and oropharynx. In contrast, $T$ stage is based on subsite involvement and is specific to each subsite for the glottic larynx, supraglottic larynx, hypopharynx, and nasopharynx.

In general, stage I or stage II disease defines a relatively small primary tumor with no nodal involvement. Stage III and stage IV cancers include large primary tumors, which may invade underlying structures and/or spread to regional nodes. Distant metastases are uncommon at presentation. In general, the survival rate of patients with locally advanced (stage III or stage IV) disease is less than $50 \%$ of the survival rate of patients with early-stage disease. 


\section{Management Approaches}

Treating the patient with $\mathrm{H} \& \mathrm{~N}$ cancer is complex. Each specific site of disease, the extent of disease, and the pathologic findings dictate the appropriate surgical procedure, radiation fields, dose and fractionation, and indications for chemotherapy. Single-modality treatment with surgery or radiotherapy is generally recommended for the approximately $40 \%$ of patients who present with early-stage disease (stage I or stage II). The 2 modalities result in similar survival in these individuals. In contrast, for the $60 \%$ of patients with locally advanced disease at diagnosis, combined modality therapy is generally recommended.

As in other NCCN practice guidelines, participation in clinical trials is emphasized as a preferred or recommended treatment option, particularly for the population with locally advanced disease. In formulating these H\&N guidelines, the panel has endeavored to make them evidence based while providing a statement of consensus as to the acceptable range of treatment options.

\section{Multidisciplinary Team Involvement}

The initial evaluation and development of a plan for treating the patient with $\mathrm{H} \& \mathrm{~N}$ cancer require a multidisciplinary team of individuals with expertise in all aspects of the special care needs of these patients. Similarly, managing and preventing sequelae of radical surgery, radiotherapy, and chemotherapy require the involvement of various health care professionals familiar with the disease. Follow-up for these sequelae should include a comprehensive $H \& N$ examination. Adequate nutritional support can help to prevent severe weight loss in patients receiving treatment for $\mathrm{H} \& \mathrm{~N}$ cancer. ${ }^{9}$ Patients should also be encouraged to stop smoking and drinking alcohol, because these habits decrease the efficacy of treatment. ${ }^{10,11}$ Programs using behavioral counseling combined with stop-smoking medications are useful for smoking cessation
(www.surgeongeneral.gov/tobacco/smokesum.htm\#Findings). Specific components of patient support and follow-up are listed in the algorithm. Pain and symptom management, social work, and case management are included in this list because of their importance in addressing the late complications of disease and its therapy. The panel also recommends referring to the NCCN Guidelines for Supportive Care.

\section{Comorbidity and Quality of Life}

\section{Comorbidity}

Comorbidity refers to the presence of concomitant disease (in addition to H\&N cancer) that may affect the diagnosis, treatment, and prognosis for the patient. ${ }^{12-14}$ Documentation of comorbidity is particularly important in oncology, because the failure to identify comorbid conditions (such as renal, heart, or liver failure) may result in inaccurate attribution of poor outcomes to the cancer. Comorbidity is known to be a strong independent predictor for mortality in H\&N cancer patients. ${ }^{14-21}$ Comorbidity has also been shown to influence costs of care, utilization, and quality of life. ${ }^{22-24}$ Numerous indices of comorbidity have been developed. Traditional indices include the Charlson index ${ }^{13}$ and the Kaplan-Feinstein index and its modifications. ${ }^{14,25}$ The Adult Comorbidity Evaluation-27 (ACE-27) is specific for H\&N cancer and has excellent emerging reliability and validity. ${ }^{26,27}$

\section{Quality of Life}

Health-related quality-of-life issues are paramount in H\&N cancer. These tumors have a tremendous effect on basic physiological functions (such as the ability to chew, swallow, and breathe), the senses (taste, smell, and hearing), and uniquely human characteristics (such as appearance and voice). In informal use, the terms health status, function, and quality of life are frequently used interchangeably; however, these terms have important distinctions. Health status describes an individual's physical, emotional, and social capabilities and limitations. Function and performance refer to how well an 
individual is able to perform important roles, tasks, or activities. On the other hand, quality of life differs, because the central focus is on the value (determined by the patient alone) that individuals place on their health status and function. ${ }^{28}$ An National Institutes of Health (NIH)sponsored conference ${ }^{29}$ recommended the use of patient-completed scales to measure quality of life. For H\&N cancer-specific issues, the 3 validated measures that have received the most widespread acceptance are: (1) the University of Washington Quality of Life scale (UW-QOL); ${ }^{30}$ (2) the European Organization for Research and Treatment of Cancer Quality of Life Questionnaire (EORTC-HN35); ${ }^{31}$ and (3) the Functional Assessment of Cancer Therapy Head and Neck module (FACT-HN). ${ }^{32} \mathrm{~A}$ clinician-rated performance scale that has also achieved widespread use is the Performance Status Scale. ${ }^{33}$ Numerous other instruments are available to measure generic cancer issues and other aspects of H\&N cancer but are beyond the scope of this discussion.

\section{Head and Neck Surgery}

\section{Resectable Versus Unresectable Disease}

The various site-specific sections of these $H \& N$ guidelines pertain to patients with resectable disease. The treatment of patients with locally advanced unresectable disease, metastatic disease, or recurrent disease is addressed in the "Advanced Head and Neck Cancer" section of these guidelines.

The term "unresectable" has resisted formal definition by H\&N cancer specialists for decades. No definition of surgical unresectability meets with universal approval. The experience of the surgeon and the support available from reconstructive surgeons, physiatrists, and prosthodontists often strongly influence recommendations. This is particularly common in institutions where few patients with locally advanced $\mathrm{H} \& \mathrm{~N}$ cancer are treated. The NCCN member institutions have teams experienced in the treatment of $\mathrm{H} \& \mathrm{~N}$ cancer and maintain the multidisciplinary infrastructure needed for reconstruction and rehabilitation. A patient's cancer is deemed unresectable if $\mathrm{H} \& \mathrm{~N}$ surgeons at NCCN member institutions doubt their ability to remove all gross tumor on anatomic grounds or if they are certain local control will not be achieved after an operation (even with the addition of radiotherapy to the treatment approach). Typically, such tumors densely involve the cervical vertebrae, brachial plexus, deep muscles of the neck, or carotid artery. Unresectable tumors (that is, those tumors unable to be removed without imposing unacceptable morbidity) should be distinguished from those tumors in patients whose constitutional state precludes an operation (even if the cancer is readily resected with few sequelae). Additionally, a subgroup of patients will refuse surgical management, but these tumors should not be deemed unresectable. Although local and regional disease may be surgically treatable, patients with distant metastases are usually treated as though the primary tumor were unresectable. Thus, patient choice or a doctor's expectations regarding cure and morbidity will influence or determine treatment.

Patients with resectable tumors who can also be adequately treated without an operation represent a very important group. Definitive treatment with radiation therapy (RT) alone or RT combined with chemotherapy may represent equivalent or preferable approaches to resection in these individuals. Although such patients may not undergo surgery, their tumors should not be labeled as unresectable. Their disease is usually far less extensive than disease that truly cannot be removed.

\section{Cervical Lymph Node Dissections}

Historically, cervical lymph node dissections have been classified as "radical" or "modified radical" procedures. The less radical procedures preserved the sternocleidomastoid muscle, jugular vein, and spinal accessory nerve. The panel prefers to classify cervical 
lymphadenectomy differently, classifying cervical lymph node dissections as either "comprehensive" or "selective."

A comprehensive neck dissection is one that removes all lymph node groups that would be included in a classic radical neck dissection. Whether the sternocleidomastoid muscle, jugular vein, or spinal accessory nerve are preserved does not affect whether the dissection is comprehensive.

Selective neck dissections have been developed based on an understanding of the common pathways for spread of H\&N cancers to regional nodes (see Figure 2) ${ }^{34,35}$ A supraomohyoid neck dissection is designed to remove the nodes most commonly involved with metastases from the oral cavity. A supraomohyoid neck dissection includes nodes found above the omohyoid muscle (level I, level II, level III, and the superior parts of level V). Similarly, a lateral neck dissection removes the nodes most commonly involved with metastases from the pharynx and larynx. A lateral neck dissection includes nodes in level II, level III, and level IV. H\&N squamous cell cancer with no clinical nodal involvement rarely presents with nodal metastasis beyond the confines of an appropriate selective neck dissection ( $<10 \%$ of the time) ${ }^{36-38}$ The chief role of neck dissections in these NCCN H\&N guidelines is to select patients for possible adjuvant radiotherapy, although there has been some enthusiasm for the use of selective neck dissections as treatment when neck tumor burden is low. In general, patients undergoing selective neck dissection should not have clinical nodal disease. In the guidelines, patients with cervical node metastases who undergo operations are generally treated with comprehensive neck dissections, because often they have disease outside the bounds of selective neck dissections.

If a complete response has been achieved after radiotherapy for N1 disease, all of the panel members are satisfied with the strategy of observing the patient. Panelists also concur that any patient with residual disease in the neck after radiotherapy should undergo a neck dissection. However, the surgeons had differing opinions regarding the management of the patient with N2 or N3 disease at initial staging who achieves a complete response to radiation. Some will observe such patients while other institutions recommend a planned neck dissection.

Many factors influence survival and locoregional tumor control in patients with H\&N cancer. In most NCCN member institutions, patients with extracapsular nodal spread and/or positive surgical margins receive adjuvant chemoradiotherapy after resection. ${ }^{39-44}$ The presence of other adverse risk factors-multiple positive nodes (without extracapsular nodal spread), vascular/lymphatic/perineural invasion, T4a primary, and oral cavity primary with positive level 4 nodes-are established indications for postoperative RT. Because patients with these features were also included in the EORTC trial that showed a survival advantage for patients receiving cisplatin concurrent with postoperative radiotherapy compared to radiotherapy alone, the panel elected to add the choice to consider RT plus chemotherapy for these features.

\section{Postoperative Management of High-Risk Disease}

The role of chemotherapy in the postoperative management of the patient with adverse prognostic risk factors has been clarified by 2 separate multicenter randomized trials ${ }^{45,46}$ and a combined analysis of data from the 2 trials for patients with high-risk cancers of the oral cavity, oropharynx, larynx, or hypopharynx. ${ }^{47}$ The US Intergroup trial R95-01 randomly assigned patients with 2 or more involved nodes, positive margins, or extracapsular spread of tumor to receive standard postoperative radiotherapy or the same radiotherapy plus cisplatin 100 $\mathrm{mg} / \mathrm{m}^{2}$ every 3 weeks for 3 doses. The European trial was designed using the same treatment but also included as high-risk factors the presence of perineural or perivascular disease and nodal involvement at levels 4 and 5 from an oral cavity or oropharynx cancer. The US trial 
demonstrated statistically significant improvement in locoregional control and disease-free survival but not overall survival, whereas the European trial found significant improvement in survival and the other outcome parameters. Note that randomized trials support several schedules for cisplatin (for example, $50 \mathrm{mg} \mathrm{IV} \mathrm{weekly}{ }^{48}$ or $6 \mathrm{mg} / \mathrm{m}^{2}$ daily $\left.^{49}\right)$, but most centers use high-dose cisplatin $\left(100 \mathrm{mg} / \mathrm{m}^{2}\right.$ every 3 weeks).

To better define risk, a combined analysis of prognostic factors and outcome from the 2 trials was performed. This analysis demonstrated that patients in both trials with extracapsular nodal spread of tumor and/or positive resection margins benefited from the addition of cisplatin to postoperative radiotherapy. For those with multiple involved regional nodes without extracapsular spread, there was no survival advantage. ${ }^{47}$ These publications form the basis for the NCCN recommendations in this updated guideline. Chemoradiation is clearly indicated for adverse risk features or extracapsular nodal spread and/or microscopic positive mucosal margins (category 1 ). The management of patients with multiple nodes only, without extracapsular spread or other adverse risk features, was discussed by the panel and postoperative RT was recommended; however, chemoradiation can be considered based on clinical judgment. The panel noted that the combined analysis was considered exploratory by the authors, because it was not part of the initial protocol design. ${ }^{47}$

\section{Head and Neck Radiotherapy}

Radiotherapy for H\&N cancer is extremely complex. Only a specially trained team consisting of a radiation oncologist, physicist, dosimetrist, and radiation technologist can achieve optimal results. In addition, modern radiotherapy equipment and techniques should be used. Anatomic, tumor, and clinical circumstances dictate the use of radiation as primary treatment or as an adjuvant to surgery in combination with chemotherapy for H\&N cancer. The NCCN radiotherapeutic guidelines are not all inclusive. Much variation in practice exists among various countries and even within different institutions in the same country.

\section{Radiation Doses}

Selection of radiation doses depends on the tumor and neck node size, location of the tumor, and clinical circumstances. In general, primary and gross adenopathy require a total of $70 \mathrm{~Gy}$ or more at a dosage of $2.0 \mathrm{~Gy} /$ day. In contrast, radiation to low-risk nodal stations in the neck requires a total of $50 \mathrm{~Gy}$ or more, also at a dosage of $2.0 \mathrm{~Gy} /$ day. Postoperative irradiation is recommended based on the tumor stage, tumor histology, and surgical findings after tumor resection. In general, postoperative RT is recommended for risk features, including multiple positive nodes (without extracapsular nodal spread) or perineural/lymphatic/vascular invasion. Higher doses of radiation (60-65 Gy) are required for microscopic disease to decrease the chances of locoregional failure because of interruption of the normal vasculature, scarring, and relative hypoxia in the tumor bed. The preferred interval between resection and postoperative RT is 6 weeks.

\section{Fractionation}

No single fractionation schedule has proven to be best for all tumors. Historically, most radiation oncology departments in the United States deliver treatment once per day, 5 days per week, at 1.8 to 2.0

Gy/fraction. Data strongly indicate some squamous cancers can grow rapidly, especially in the face of cell depletion. The upper dose of 2.0 Gy/fraction, delivering 1000 cGy or greater per week, is now the most commonly used dose among the NCCN member institutions. Thus, the guidelines have been revised to indicate that the dose of $2.0 \mathrm{~Gy} /$ fraction is preferred, with the exception of salivary gland tumors, which may have slower cell kinetics. ${ }^{50-54}$ External radiation doses exceeding $75 \mathrm{~Gy}$ at conventional fractionation of 1.8 to $2.0 \mathrm{~Gy} /$ day may lead to unacceptable normal tissue injury. 
Most of the published studies with concurrent chemoradiation have used conventional fractionation at 2.0 Gy per fraction to 70 Gy or more in 7 weeks with single-agent cisplatin given every 3 weeks at 100 $\mathrm{mg} / \mathrm{m}^{2}$. Use of other fraction sizes (for example, $1.8 \mathrm{~Gy}$, conventional), multiagent chemotherapy, or altered fractionation with chemotherapy has been evaluated, but there is no consensus on the optimal approach. In general, the use of concurrent chemoradiation carries a high toxicity burden, and altered fractionation or multiagent chemotherapy will likely further increase the toxicity burden. For any chemotherapeutic approach, close attention should be paid to published reports for the specific chemotherapy agent, dose, and schedule of administration. Chemoradiation should be performed by an experienced team and should include substantial supportive care.

Altered fractionation includes accelerated treatment delivering more than 1000 cGy per week and hyperfractionation. The biological rationale for using hyperfractionation is based on the discovery by Withers and colleagues of a large, consistent difference in repair capacity of late and early responding tissues. ${ }^{55,56}$ Accelerated schedules attempt to compensate for rapid tumor proliferation by compressing the time-dose schedule. A number of phase II trials have suggested an advantage to the use of altered fractionation schemes in various $\mathrm{H} \& \mathrm{~N}$ cancers. ${ }^{57}$

Two large, randomized clinical trials have reported improved locoregional control using altered fractionation. The European Organization for Research and Treatment of Cancer (EORTC) protocol 22791 compared hyperfractionation (1.15 Gy twice daily, or 80.5 Gy over 7 weeks) with conventional fractionation (2 Gy once daily, or 70 Gy over 7 weeks) in the treatment of T2,T3,N0-1 oropharyngeal carcinoma. At 5 years, there was a statistically significant increase in local control in the hyperfractionation arm (38\% versus $56 \% ; P=.01)$ and no increase in late complications. ${ }^{58} \mathrm{~A}$ long-term follow-up analysis has also demonstrated a small survival advantage for hyperfractionation $(P=.05) .{ }^{59}$ Another EORTC protocol (22851) compared accelerated fractionation (1.6 Gy 3 times daily, or 72 Gy over 5 weeks) with conventional fractionation (1.8-2.0 Gy once daily, or 70 Gy over 7-8 weeks) in various intermediate to advanced H\&N cancers (excluding cancers of the hypopharynx). Patients in the accelerated fractionation arm did significantly better with regard to locoregional control $(P=.02)$ at 5 years. Disease-specific survival showed a trend $(P=.06)$ in favor of the accelerated fractionation arm. Acute and late toxicity were increased in this fractionation arm, however, raising questions about the net advantages of accelerated fractionation. ${ }^{60}$

In the United States, the Radiation Therapy Oncology Group (RTOG) reported the initial 2-year results and subsequent mature results (after a median follow-up of 8.5 years) of a large phase III clinical trial (protocol 90-03) comparing hyperfractionation and 2 variants of accelerated fractionation with standard fractionation. ${ }^{61,62}$

After 2 years of follow-up, both accelerated fractionation with a concomitant boost (AFX-C) and hyperfractionation were associated with improved locoregional control and disease-free survival compared with standard fractionation. However, acute toxicity was increased. No significant difference was demonstrated in the frequency of grade 3 or worse late effects reported at 6 to 24 months after treatment start, among the various treatment groups. Long-term follow-up confirmed a statistically significant improvement in locoregional control with either AFX-C or hyperfractionation compared to standard fractionation. However, neither disease-free survival nor overall survival were significantly improved. Severe late toxicity was more frequently observed with AFX-C. A meta-analysis of updated patient data analyzing the effect of hyperfractionated or accelerated radiotherapy on survival of patients with head and neck cancer was recently published. This meta-analysis included 15 randomized trials that had standard 
fractionation as the control group. An absolute survival benefit of 3.4\% at 5 years (HR 0.92; $95 \% \mathrm{Cl}, 0.86-0.97 ; P=.003)$ was reported. This benefit, however, was limited to patients younger than 60 years of age.$^{63}$ Consensus regarding altered fractionation schedules with concomitant boost or hyperfractionation for stage III or IV oral cavity, oropharynx, supraglottic larynx, and hypopharyngeal squamous cell cancers has not yet emerged among NCCN member institutions. ${ }^{63-65}$

\section{Brachytherapy}

Brachytherapy is used less often because of improved local control obtained with concurrent chemo/RT. However, brachytherapy still has a role primarily for lip cancer, cancer of the oral cavity, and oropharynx. Several European and North American medical centers have had extensive experience with brachytherapy. ${ }^{66-74}$ The success of brachytherapy techniques is partly dependent on the training, experience, and skills of the implant team.

\section{Intensity-Modulated Radiation Therapy}

The intensity of the radiation beam can be modulated in order to decrease doses to normal structures without compromising the doses to the target. Intensity-modulated radiation therapy (IMRT) is an advanced form of 3-D conformal RT with enormous potential to precisely target and to enable escalation of the radiation dose; the net effect is decreased radiation exposure to normal structures. During the past several years, an exponential growth has occurred in the use of IMRT for various malignancies, in particular, prostate and H\&N cancers.

Several institutions have conducted phase II studies to explore the use of beam modulation in H\&N cancer. The objective data from these institutions consistently show a decrease in acute and late toxicities without compromising tumor control. ${ }^{75-82}$ However, no phase III studies have been done to substantiate the results from phase II studies.
RTOG 0022 and RTOG 0225 are single-arm studies exploring the feasibility of IMRT in the treatment of oropharyngeal and nasopharyngeal cancer. ${ }^{83,84}$ These trials are currently ongoing. At present, IMRT is not the standard of care for the treatment of H\&N cancers; however, selected patients may benefit from this new technology if they are treated in centers that have expertise in IMRT.

Three-dimensional conformal techniques may be used depending on the stage, tumor location, physician training/experience, and available physics support. IMRT techniques are an area of active investigation among the NCCN institutions and others. ${ }^{85-88}$ Target delineation and optimal dose distribution require special training in H\&N imaging, a thorough understanding of patterns of disease spread, and special training in IMRT techniques. Standards for target definition, dose specification, fractionation (with and without concurrent chemotherapy), and normal tissue constraints should emerge within the next few years.

\section{Paranasal Tumors (Maxillary and Ethmoid Sinus Tumors)}

Tumors of the paranasal sinuses are rare and often asymptomatic until late in the course of their disease. Although the most common histology for these tumors is squamous cell carcinoma, multiple histologies have been reported including sarcoma (excluding rhabdomyosarcoma), lymphoma, adenocarcinoma, esthesioneuroblastoma (also known as olfactory neuroblastoma), small cell neuroendocrine and undifferentiated carcinoma (sinonasal undifferentiated carcinoma [SNUC]) ${ }^{89-91}$ Locoregional control and incidence of distant metastasis are dependent on both T stage and tumor histology. However, T stage remains the most reliable predictor of survival and local regional control (see $\underline{\text { Table 5)}}$. 


\section{Management of Ethmoid Sinus Cancer}

Patients with early-stage cancer of the ethmoid sinus are asymptomatic. These neoplasms are often found after a routine nasal polypectomy or during the course of a nasal endoscopic procedure. For a patient with gross residual disease who has had a nasal endoscopic surgical procedure, the preferred treatment is complete surgical excision of the residual tumor. This procedure often entails an anterior craniofacial resection to remove the Cribriform plate and to ensure clear surgical margins. Most patients affected by ethmoid sinus cancer present after having had an incomplete excision. The patient who is diagnosed after incomplete excision (for example, polypectomy, endoscopic surgical procedure)—and has no documented residual disease on physical examination, imaging, and endoscopy-should be treated in a similar fashion if feasible. If no adverse pathologic factors are found, this treatment ensures clear surgical margins and obviates the need for postoperative radiotherapy. However, RT may be used as definitive treatment in patients if pre-biopsy imaging studies and nasal endoscopy demonstrate that the superior extent of the disease does not involve the skull base. Systemic therapy should be part of the overall treatment for patients with SNUC, esthesioneuroblastomas, or small cell neuroendocrine histologies. ${ }^{92-96}$

\section{Treatment of Maxillary Sinus Tumors}

Complete surgical resection for all $\mathrm{T}$ stages followed by postoperative therapy remains a cornerstone of treatment. In addition, RT or chemotherapy/RT (category 2B) should be considered for T1-2, N0 tumors with perineural invasion. Neck dissection is indicated in the treatment of the clinically positive neck. Finally, a combination of chemotherapy and RT or definitive RT alone (without chemotherapy) may be used to treat surgically unresectable disease. Patients with maxillary sinus tumors who have adverse characteristics (for example, positive margins, perineural invasion, or extracapsular nodal spread) should receive surgical resection (if possible) followed by
chemotherapy/RT to the primary and neck (category 2B). Participation in clinical trials is favored for patients with malignant tumors of the paranasal sinuses.

\section{Salivary Gland Tumors}

Salivary gland tumors can arise in the major salivary glands (parotid, submaxillary, or sublingual salivary glands) or in one of the minor salivary glands, which are widely spread throughout the aerodigestive tract. Many minor salivary gland tumors are located on the hard palate. Even though many salivary gland tumors are generally benign, approximately $20 \%$ of the parotid gland tumors are malignant; the incidence of malignancy in submandibular and minor salivary gland tumors is approximately $50 \%$ and $80 \%$, respectively. These malignant tumors constitute a broad spectrum of histologic types, including mucoepidermoid, acinic, adenocarcinoma, adenoid cystic carcinoma, malignant myoepithelial tumors, and squamous carcinoma. The primary diagnosis of squamous carcinoma of the parotid gland is rare; however, the parotid is a frequent site of metastasis from skin cancer. ${ }^{97}$

Prognosis and tendency to metastasize vary among these histologic types. Major prognostic factors are histologic grade, tumor size, and local invasion (see Table 4).

\section{Treatment}

The major therapeutic approach for salivary gland tumors is adequate and appropriate surgical resection. Surgical intervention requires careful planning and execution, particularly in parotid tumor surgery because of the presence of the facial nerve within the gland, which should be preserved if the nerve is not directly involved by the tumor. Most of the parotid gland tumors are located in the superficial lobe, and if the facial nerve is functioning preoperatively, the nerve can be preserved in most patients. The facial nerve should be sacrificed if there is preoperative facial nerve involvement with facial palsy or if there is direct invasion of the tumor into the nerve where the tumor 
cannot be separated from the nerve. Malignant deep lobe parotid tumors are quite rare; however, they are generally a challenge for the surgeon where the patient may require superficial parotidectomy and identification and retraction of the facial nerve to remove deep lobe parotid tumor.

Most malignant deep lobe parotid tumors will require postoperative RT because of the limitations of surgical margins in the resection of these tumors. RT is used in an adjuvant setting for tumors with adverse characteristics; chemotherapy/RT (category $2 \mathrm{~B}$ ) can also be considered. Adjuvant radiotherapy is indicated after resection if adverse characteristics are present, such as positive or close margins, neural or perineural infiltration (often seen with adenoid cystic carcinomas), or lymph node metastases. Adjuvant RT is also recommended if the tumor is intermediate or high grade, or if lymphovascular invasion or extracapsular spread is present. ${ }^{97}$

For unresectable tumors, RT alone (without chemotherapy) is used as definitive treatment; however, chemoradiation (cisplatin) is also an option (category 2B). The panel was not in agreement regarding chemoradiation, because there are no published trials of this approach for unresectable salivary gland tumors. Chemotherapy may be used for palliation in advanced disease. Various agents (for example, paclitaxel) and combinations (for example, cisplatin, doxorubicin,

cyclophosphamide; carboplatin and paclitaxel) have been shown in small series to be active for some salivary gland malignant histologies.

\section{Carcinoma of the Lip}

The guidelines for squamous cell carcinoma of the lip generally follow accepted clinical practice patterns established over several decades. No randomized clinical trials have been conducted that can be used to direct therapy. In general, treatment strategies are determined by anticipated functional and cosmetic results. The incidence of lymph node metastases, especially in early-stage lower lip cancer, is low, averaging less than $10 \%$. The risk of lymph node metastases is related to the location, size, and grade of the primary tumor. Elective neck dissection or neck irradiation can be avoided in patients with earlystage disease and a clinically negative neck. Treatment recommendations are based on clinical stage, medical status of the patient, and patient preference.

\section{Workup and Staging}

The workup for patients with squamous cell carcinoma of the lip consists of a physical examination, biopsy, and chest imaging. A dental Panorex (panoramic x-ray) and computerized tomographic (CT) scan or magnetic resonance imaging (MRI) are done if bone invasion is suspected. The AJCC TNM staging system reflects tumor size, extension, and nodal disease (see Table 1). This system does predict the risk for local recurrence. The location of the primary tumor also is predictive. Tumors in the upper lip and commissural areas have a higher incidence of lymph node metastases at the time of diagnosis. Systemic dissemination is rare, occurring in approximately $10 \%$ to $15 \%$ of patients, most often in those with uncontrolled locoregional disease.

\section{Treatment of the Primary}

The treatment of lip cancer is governed by the stage of the disease. The choice of a local treatment modality is based on the expected functional and cosmetic outcome. In early-stage cancers, surgery and radiation are equivalent options in terms of local control. Some very small or superficial cancers are managed more expeditiously with a surgical excision without resultant functional deformity or an undesired cosmetic result. On the other hand, a superficial cancer that occupies most of the lower lip, for example, would be best managed with RT. Some advanced lip cancers can cause a great deal of tissue destruction and secondary deformity. Surgery is a more viable option in this clinical setting. Surgery is also the local modality of choice for 
advanced cancers with extension into the bone. Patients with resectable T3, N0; T4a, N0; or any T, N1-3 disease who are a poor surgical risk can be treated with definitive RT (with or without brachytherapy) or with chemotherapy/RT.

\section{Management of the Neck}

The management of the neck is also governed by stage, but the location of the tumor should also be taken into account. For example, the lymphatics of the upper lip are very extensive. Thus, tumors in this location are more apt to spread to deep superior jugular nodes. The position of the tumor along the lip also can be helpful in predicting the pattern of lymph node spread. A midline location can place a patient at higher risk for contralateral disease. For patients with advanced disease (T3, T4a) and an NO neck, a unilateral or bilateral selective neck dissection should be considered. When a patient presents with palpable disease, care is taken to ensure all appropriate nodal levels are dissected.

\section{Radiation}

Radiotherapy, when used as definitive treatment, may consist of external-beam RT or brachytherapy alone or in combination, depending on the size of the tumor. The dose required also depends on tumor size, but doses of 66 Gy or more are adequate to control the disease. For T1-2, N0 lesions, the total dose of external-beam RT may be decreased to $50 \mathrm{~Gy}$ or more when given in conjunction with brachytherapy. In the adjuvant setting, doses of 60 Gy or more are required, depending on the pathologic features. In both definitive and adjuvant settings, the neck is treated with doses that address adverse features, such as positive margins or invasion (perineural, vascular, and/or lymphatic)

\section{Follow-up/Surveillance}

Follow-up for patients with treated cancers of the lip relies solely on periodic physical examinations every 1 to 3 months during year 1 , every 2 to 4 months during year 2 , every 4 to 6 months during years 3 to 5 , and every 6 to 12 months thereafter.

\section{Cancer of the Oral Cavity}

The oral cavity includes the following subsites: buccal mucosa, upper and lower alveolar ridge, retromolar trigone, floor of the mouth, hard palate, and anterior two thirds of the tongue. There is a rich lymphatic supply to the area, and initial regional node dissemination is to nodal groups at level I, level II, and level III.

Regional node involvement at presentation is evident in approximately $30 \%$ of patients, but the risk varies according to subsite. For example, primaries of the alveolar ridge and hard palate infrequently involve the neck, whereas occult neck metastasis is common (50\% to 60\%) in patients with anterior tongue cancers. In general, all patients undergo treatment of the neck with either unilateral or bilateral selective neck dissection or if primary RT is chosen for treatment of T1-2, N0 disease, at least $50 \mathrm{~Gy}$ is given to the neck at risk.

\section{Workup and Staging}

Imaging studies to evaluate mandibular involvement and a careful dental evaluation (including Panorex , as indicated) are particularly important for staging (see Table 1) and planning therapy for oral cavity cancers in addition to a physical examination, biopsy, and chest imaging.

\section{Treatment}

Surgery and RT represent the standards of care for early-stage and locally advanced resectable lesions in the oral cavity. The specific treatment is dictated by the TN stage and, if $\mathrm{NO}$ at diagnosis, by the risk 
of nodal involvement. Multidisciplinary team involvement is particularly important for this site because of the critical physiologic functions of mastication, deglutition, and articulation of speech, which may be affected. Most panelists prefer surgical therapy for resectable oral cavity tumors. Advances in reconstruction using microvascular techniques have led to improved functional outcomes for patients with locally advanced disease.

Postoperative chemotherapy/RT is recommended (category 1 ) for all patients with resected oral cavity cancers with the adverse pathologic features of extracapsular nodal spread and/or a positive mucosal margin $^{45-47}$ (category 1). For other risk features-such as pT3 or pT4 primary, N2 or N3 nodal disease, nodal disease in levels IV or V, or perineural invasion or vascular embolism-clinical judgment should be utilized in the consideration of adding chemotherapy to RT or treating with RT alone. The concept of organ preservation using chemotherapy in the initial management of locally advanced resectable disease has not been studied in trials specifically designed for this site.

\section{Follow-up/Surveillance}

Follow-up for patients with treated cancers of the oral cavity consists of periodic physical examinations, chest imaging as clinically indicated, and, if the thyroid was irradiated, measurement of thyrotropin (TSH) every 6 to 12 months. Speech, hearing, \& swallowing evaluation and rehabilitation may be useful, as indicated. Smoking cessation counseling and dental follow-up are also recommended.

\section{Cancer of the Oropharynx}

The oropharynx includes the base of the tongue, tonsils, soft palate, and posterior pharyngeal wall. The oropharynx is extremely rich in lymphatics. Depending on the subsite involved, $15 \%$ to $75 \%$ of patients present with lymph node involvement. Efforts to improve the outcome of patients with locally advanced disease are ongoing. Participation in clinical trials is strongly recommended.

\section{Workup and Staging}

A multidisciplinary consultation is encouraged. Accurate staging depends on a thorough physical examination coupled with appropriate imaging studies. CT with contrast or MRI is recommended for the primary and the neck; chest imaging is also recommended.

Examination of the $\mathrm{H} \& \mathrm{~N}$ region should include an examination under anesthesia with endoscopy. Bronchoscopy and esophagoscopy are also recommended because of the relative frequency of simultaneous second primaries. A dental evaluation is recommended, with Panorex studies as indicated. Speech and swallowing evaluation may be useful, as indicated. Testing for high-risk oncogenic HPV types (HPV 16, -18, $-31,-33,-35$ ) is recommended (using either polymerase chain reaction [PCR] or in situ hybridization [ISH]) because of the strong association with oropharyngeal squamous cell carcinoma and the increasing incidence of cancers of the lingual and palatine tonsils. ${ }^{4,98,99}$ In patients with oropharyngeal carcinoma, recent data indicate that those with HPV-associated cancers have improved response to treatment and improved survival when compared with HPV-negative tumors. ${ }^{100}$

\section{Treatment}

The treatment algorithm has been divided into 3 staging categories: (1) T1-2, N0-1; (2) T3-4a, N0; and (3) T3-4a, N+ or any T, N2-3. Earlystage (T1-2, N0-1) oropharyngeal cancers may be treated with primary surgery including neck dissection, as indicated, or with definitive radiotherapy (category $2 \mathrm{~B}$ ). The panel members strongly disagreed about the third option of RT plus systemic therapy (category 3) (see ORPH-2). Surgery is also indicated for salvage in cases of residual or recurrent disease after definitive radiotherapy. Radiotherapy alone is appropriate management for patients with a T1 or T2 primary and N1 disease. For patients treated surgically who have adverse pathologic 
features that warrant post-operative RT, the optimal time interval between resection and RT is not more than 6 weeks. Adjuvant chemotherapy/RT is recommended (category 1 ) for adverse pathologic features of extracapsular nodal spread and/or positive mucosal margin. ${ }^{101}$ For other risk features-such as pT3 or pT4 primary, N2 or N3 nodal disease, nodal disease in levels IV or $\mathrm{V}$, or perineural invasion or vascular embolism—clinical judgment should be utilized in the consideration of adding chemotherapy to RT or treating with RT alone.

For advanced resectable disease (T3-4a, any $\mathrm{N}$ or any $\mathrm{T}, \mathrm{N} 2-3)$, there are 3 treatment approaches in the algorithms (see ORPH 3 and ORPH 4), in addition to enrollment in a multimodality clinical trial that includes function evaluation. The three approaches are : (1) concurrent systemic therapy/RT (for example, cisplatin [category 1] preferred) (salvage surgery is used for managing residual or recurrent disease); ${ }^{101}$ (2) surgery with neck dissection and reconstruction, as indicated, followed by radiotherapy with or without chemotherapy as dictated by the presence of pathologic adverse features; or (3) induction chemotherapy followed by chemo/RT (category 3) for which there was strong disagreement among panel members. ${ }^{101-106}$

For the concurrent systemic therapy/RT approach, all patients are evaluated at completion of treatment for response in the primary site and in the neck. For patients with nodal involvement at initial staging who achieve a complete response in the primary and the neck, subsequent management is based on initial nodal stage. Patients who had N1 disease are observed, while patients who had N2 or N3 disease can be either observed or undergo a planned neck dissection. This is an area of controversy among NCCN institution surgeons. However, if residual neck disease is present (and the primary is controlled), there is uniform agreement to proceed to neck dissection. Patients with residual tumor in the primary should be offered salvage surgery with neck dissection as indicated.

Concurrent systemic therapy/RT with cisplatin (category 1 ) is preferred for treatment of locally advanced (T3-4a or N2-3) cancer of the oropharynx. The use of induction chemotherapy added to chemoradiotherapy (termed sequential therapy) is an area of controversy among the NCCN panelists. Most randomized trials of induction chemotherapy followed by radiotherapy or surgery (which were published in the 1980s and 1990s) did not demonstrate a survival advantage. Induction chemotherapy had no effect on local control; however, in many trials, it did reduce the distant metastatic rate. A rationale for reevaluating induction chemotherapy added to concurrent chemotherapy/RT is to reduce distant metastases as a site of failure now that improved local control can be achieved with concurrent chemotherapy/RT. Results from 3 phase III trials-that compared induction cisplatin plus infusional 5-FU with or without the addition of a taxane (docetaxel or paclitaxel)—showed significantly improved outcomes (response rates, disease-free survival or overall survival depending on the trial) for patients in the 3 drug induction group compared to those receiving 2 drugs (cisplatin plus 5-FU). ${ }^{104,106,107}$ Taken together, these results show that a more effective triplet combination has been identified compared to the standard of cisplatin plus 5-FU used in the induction trials of the 1980s and 1990s. However, a survival advantage over concurrent chemoradiation has yet to be demonstrated. The NCCN panel uniformly agreed that clinical trials should be performed to directly answer the question of whether or not induction chemotherapy added to systemic therapy/RT improves survival in patients with locally advanced cancer of the oropharynx and other specified sites. Such trials are in progress, and the panel members uniformly agreed that patients should be enrolled in these trials. The panel members differed in their opinion as to whether or not this treatment should be considered a standard treatment option. A 
small minority of panel members do advocate this approach (see ORPH-3). This disagreement is reflected by a category 3 recommendation in the algorithms.

Altered fractionation is preferred when radiotherapy is used definitively for selected T1, N1 or T2, N0-1 tumors. For patients not receiving concurrent chemoradiation, altered fractionation is preferred. The recommended schedules are: (1) concomitant boost accelerated radiotherapy consisting of 72 Gy delivered over 6 weeks using 1.8 Gy/fractions to the large volume and $1.5 \mathrm{~Gy}$ boost as the second daily fraction 6 hours later during the last 12 treatments to a smaller volume; or (2) hyperfractionation consisting of 81.6 Gy given in 7 weeks with 1.2 Gy/fractions twice daily 6 hours apart. This change from standard radiotherapy for large lesions was made on the basis of the results of the RTOG 9003 protocol, which detected a local control advantage for patients who were treated with hyperfractionation and concomitant boost versus those treated with standard fractionation or accelerated fractionation with a break in the treatment schedule. ${ }^{61,62}$ Increased acute toxicity was demonstrated in both altered fractionation schedules when compared with standard radiotherapy. The concomitant boost schedule resulted in prolongation of acute symptoms 6 to 24 months after the initiation of treatment, but no significant difference was demonstrated in the frequency of late effects among schedules. In addition to the RTOG trial, 4 other randomized trials have demonstrated improved outcomes with hyperfractionation. ${ }^{58,108}$

\section{Salvage Surgery}

Patients with advanced carcinoma of the oropharynx who undergo nonsurgical treatment, such as concurrent chemotherapy and RT, need very close follow-up both to evaluate for local recurrence and to assess for ipsilateral or contralateral neck recurrence. The patients who do not respond completely to chemotherapy/RT require salvage surgery to the primary and the neck. However, all panelists emphasized that it may be difficult to detect local or regional recurrence due to radiation-related tissue changes and, this may result in a delayed diagnosis of persistent or recurrent disease. The panelists also emphasized the increased risk of complications when salvage surgery is attempted. Additionally, laryngectomy is occasionally required to obtain clear surgical margins or to prevent aspiration in patients with advanced oropharyngeal cancer. Some of these patients may require microvascular free flap reconstruction to cover the defects at the primary site. The patients undergoing neck dissection may develop complications related to delayed wound healing, skin necrosis, or carotid exposure. The patients requiring salvage laryngectomy may have high incidence of pharyngocutaneous fistula and may require either a free flap reconstruction of the laryngopharyngeal defect or if the pharynx can be closed primarily, buttressing the suture line with myocutaneous flap.

\section{Follow-up/Surveillance}

The follow-up of patients treated for oropharyngeal cancer continues to rely on physical examination. Chest imaging is recommended as clinically indicated as surveillance for second primary tumors. Posttreatment baseline imaging (3-6 months) of the primary and neck is recommended for disease that is difficult to follow (ie, patients who have had chemoradiation not surgery); repeat imaging if indicated by signs/symptoms on physical examination. If PET is used for follow-up, the first scan should be performed at not less than 12 weeks after treatment to reduce the false-positive rate. Patients whose thyroid gland has been irradiated should have TSH levels monitored every 6 to 12 months. Speech, hearing, \& swallowing evaluation and rehabilitation and dental evaluation should be done as indicated. Smoking cessation counseling is also recommended.

\section{Cancer of the Hypopharynx}

The hypopharynx extends from the superior border of the hyoid bone to the lower border of the cricoid cartilage and is essentially a muscular, 
lined tube extending from the oropharynx to the cervical esophagus. For staging purposes, the hypopharynx is divided into 3 areas: (1) the pyriform sinus (the most common site of cancer in the hypopharynx); (2) the lateral and posterior pharyngeal walls; and (3) the postcricoid area.

\section{Workup and Staging}

A multidisciplinary consultation is encouraged. Accurate staging depends on a thorough physical examination coupled with appropriate imaging studies, such as CT with contrast or MRI of the primary and neck and chest imaging. Examination of the H\&N region should include an examination under anesthesia with endoscopy. Bronchoscopy and esophagoscopy are also recommended because of the relative frequency of simultaneous second primaries. A dental evaluation is recommended. Speech and swallowing evaluation should be performed in most patients.

At the time of diagnosis, approximately $60 \%$ of patients with cancer of the hypopharynx have locally advanced disease with spread to regional nodes. Furthermore, autopsy series have shown a high rate of distant metastases (60\%) involving virtually every organ. ${ }^{109}$ Thus, the prognosis for patients with cancer of the hypopharynx is quite poor. Despite standard radical surgery and radiotherapy, the persistent or recurrent locoregional disease and distant dissemination contribute to the poor outcome for these patients.

\section{Treatment}

Patients with resectable disease are divided into 2 groups: those patients with early-stage cancer (T1, N0; small T2, N0) who do not require a total laryngectomy and those patients with advanced resectable cancer ( $\mathrm{T} 1, \mathrm{~N}+; \mathrm{T} 2-4 \mathrm{a}$, any $\mathrm{N}$ ) who do require laryngectomy. The surgery and radiotherapy options for the former group (see HYPO-2) represent a consensus among the panel members. For patients treated initially with definitive RT (without chemotherapy), surgery is indicated for residual neck disease. For patients with a complete response of the neck, observation is recommended.

Patients with more advanced disease (defined as $\mathrm{T} 1, \mathrm{~N}+; \mathrm{T} 2-3$, any $\mathrm{N}$ ) (see Table 2) requiring total laryngectomy and partial or total pharyngectomy may be managed with 3 approaches in addition to enrollment in a multimodality clinical trial that includes function evaluation: (1) induction chemotherapy followed by definitive RT if a complete response was achieved at the primary site (category 1$)^{110}$ or followed by concurrent chemoradiation if a partial response was achieved at the primary site (category $2 \mathrm{~B}$ ) or followed by surgery if the response to induction chemotherapy was less than partial; (2) surgery with neck dissection and post-operative radiation or chemoradiation as dictated by pathologic risk features; or (3) concurrent chemotherapy/RT cisplatin preferred (category $2 \mathrm{~B}$ ). Note that the panel uniformly supports the recommendation of induction chemotherapy (category 1 ) followed either by definitive RT (category 1 ) or by chemoradiation (category 2B) based upon the response achieved at the primary site for patients with (1) $\mathrm{T} 1, \mathrm{~N}+$, or (2) T2-3, any $\mathrm{N}$ disease. ${ }^{110}$ The standard induction regimen is docetaxel, cisplatin, and 5-FU (TPF). ${ }^{106,107,111}$ Given the functional loss resulting from this surgery and the poor prognosis, participation in clinical trials is emphasized.

The recommendation of the induction chemotherapy (cisplatin and 5 -FU)/definitive radiotherapy option is based on the results of an EORTC randomized trial. ${ }^{110}$ This trial enrolled 194 eligible patients with stage II, stage III, or stage IV resectable squamous cell carcinoma of the pyriform sinus (152 patients) and aryepiglottic fold (42 patients), excluding patients with T1 or N2c disease. Patients were randomly assigned either to laryngopharyngectomy and postoperative radiotherapy, or to chemotherapy with cisplatin and 5-FU for a maximum of 3 cycles, followed by definitive radiotherapy. In contrast to 
a similar regimen used for laryngeal cancer, a complete response to induction chemotherapy was required in order to proceed with definitive radiotherapy. The published results showed equivalent survival, with median survival duration and 3-year survival rate of 25 months and $43 \%$, respectively, for the surgery group versus 44 months and $57 \%$, respectively, for the induction chemotherapy group. A functioning larynx was preserved in $42 \%$ of patients who did not undergo surgery. Local or regional failure rates did not differ between the surgery-treated patients and chemotherapy-treated patients, although the chemotherapy recipients did demonstrate a significant reduction in distant metastases as a site of first failure $(P=.041)$. Adherence to the requirements for complete response to chemotherapy and for inclusion of only patients with the specified TN-stage are emphasized.

As noted in the algorithm, surgery is recommended if less than a partial response occurs after 3 cycles of induction chemotherapy. In this situation, or when primary surgery is the selected management path, postoperative chemotherapy/RT is recommended (category 1 ) for adverse pathologic features of extracapsular nodal spread and/or positive mucosal margin. For other risk features—such as pT3 or pT4 primary, N2 or N3 nodal disease, nodal disease in levels IV or V, or perineural invasion or vascular embolism-clinical judgment should be utilized in the consideration of adding chemotherapy to RT or treating with RT alone.

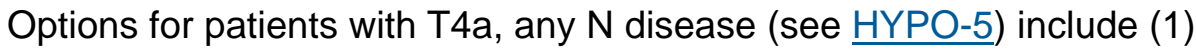
surgery plus comprehensive neck dissection (preferred) followed by adjuvant chemotherapy/RT (category 1); (2) multimodality clinical trials that include function evaluation; (3) concurrent systemic therapy/RT (category 3); or induction chemotherapy followed by chemotherapy/RT or RT (category 3).

\section{Follow-up/Surveillance}

The recommended schedule of follow-up evaluations for patients with cancer of the hypopharynx (see HYPO-5) is the same as for patients with cancer of the oropharynx.

\section{Occult Primary Cancer}

When patients present with metastatic tumor in a neck node and no primary site can be identified after appropriate investigation, the tumor is defined as an "occult" or unknown primary cancer; this is an uncommon disease, accounting for about $5 \%$ of patients presenting to referral centers. While patients with very small tonsil and tongue base cancers frequently present with enlarged neck nodes and are classified as an "unknown primary", most will be diagnosed by directed biopsy and tonsillectomy. H\&N cancer of unknown primary site is a highly curable disease. After appropriate evaluation and treatment, most patients experience low morbidity and many will be cured. The primary tumor becomes apparent on follow-up only in a few cases. Patients and oncologists are often concerned when the primary cancer cannot be found. This concern may lead to intensive, fruitless, and costly diagnostic maneuvers.

Most patients older than 40 years who present with a neck mass prove to have metastatic cancer. The source of the lymphadenopathy is almost always discovered in the course of a complete H\&N examination, which should be performed on all patients with neck masses before other studies are initiated. The following should be assessed during office evaluation: 1) antecedent history of malignancy and 2) prior excision, destruction, or regression of cutaneous lesions.

\section{Workup}

When patients present with a neck mass, fine-needle aspiration (FNA) should be the first study undertaken. Needle aspiration generally guides 
management and treatment planning. Core or open biopsy should be avoided, because it may alter or interfere with subsequent treatment.

When a needle biopsy demonstrates squamous cell carcinoma, adenocarcinoma, or anaplastic epithelial cancer and no primary site has been found, additional studies are needed. HPV-16 and Epstein Barr Virus encoded RNA (EBER) testing are suggested for squamous cell or undifferentiated histology. ${ }^{98,99,112-114}$

Nasopharyngolaryngoscopy, chest imaging, and either CT scan with contrast or MRI with gadolinium should be performed. A fused PET/CT scan should only be done if other tests do not reveal a primary. PET can be used to confirm clinical impressions, detect an unknown primary, and for surveillance. Other imaging studies have very low yield and should not be undertaken.

If the FNA proves nondiagnostic, then repeat FNA, core biopsy, or open biopsy may be needed. Open biopsy should not be performed unless the patient is prepared for definitive surgical management of the malignancy documented in the operating room. This management may entail a formal neck dissection. Therefore, an open biopsy of an undiagnosed neck mass should not be undertaken lightly, and patients should be thoroughly apprised of the potential sequelae.

When the imaging studies and thorough office examination (including examination of the nasopharynx, oropharynx, larynx, and hypopharynx and attention to the skin) do not reveal a primary tumor, then an examination under anesthesia should be performed. Mucosal sites should be inspected and examined. Appropriate endoscopies with directed biopsies of likely primary sites are recommended, but they seldom disclose a primary cancer. Many primary cancers are identified after tonsillectomy. However, the clinical significance of such tumors is uncertain. When patients have been treated without tonsillectomy, only a few develop a clinically significant primary tumor.

\section{Treatment}

Comprehensive neck dissection (including level I-III) is recommended for all patients with adenocarcinoma. If the metastatic adenocarcinoma presents high in the neck, parotidectomy may be included with the neck dissection. NCCN member institutions have major disagreements about the management of patients with poorly differentiated or nonkeratinizing squamous cell, anaplastic cancer of unknown primary site, or other uncommon histologies. Some members believe such patients should be managed with comprehensive neck dissection (levels I-V), whereas others believe the following options can be used (all the following are category 3): (1) chemoradiation; (2) primary RT; or (3) induction chemotherapy followed by chemoradiation.

If an N1 node was excised in an open biopsy, then most NCCN institutions recommend radiation that encompasses the potential primary sites as determined by the neck node levels involved and both necks. Some institutions would radiate the neck only (category 3 ). When a neck dissection is performed, postoperative radiation and concurrent chemotherapy are recommended by all institutions if extracapsular nodal spread was present or if the patient presented with N2 or N3 disease. Some NCCN institutions would radiate the neck only (category 3 ), whereas most institutions would also radiate the likely occult primary sites based on the level of nodes involved;

chemotherapy/RT to the likely primary site and the neck is also an option (category 2B). Extending the radiation field to encompass all possible mucosal primary sites is controversial and the source of much disagreement. There is little evidence to support a survival benefit from radiation to all possible primary sites.

\section{Cancer of the Larynx}

The larynx is divided into 3 regions: supraglottis, glottis, and subglottis. The distribution of cancers is as follows: $30 \%$ to $35 \%$ in the supraglottic region, $60 \%$ to $65 \%$ in the glottic region, and $5 \%$ in the subglottic 
region. The AJCC staging classification for laryngeal primary tumors is determined by the number of subsites involved, vocal cord mobility, and the presence of metastases (see Table 3).

The incidence and pattern of metastatic spread to regional nodes varies with the primary region. More than $50 \%$ of patients with supraglottic primaries present with spread to regional nodes because of an abundant lymphatic network that crosses the midline. Bilateral adenopathy is not uncommon with early-stage primaries. Thus, supraglottic cancer is often locally advanced at diagnosis. In contrast, the lymphatic drainage of the glottis is sparse and early-stage primaries rarely spread to regional nodes. Because hoarseness is an early symptom, most glottic cancers are in an early stage at diagnosis. Thus, glottic cancers have an excellent cure rate-in the range of $80 \%$ to $90 \%$. As with other cancers of the H\&N, nodal involvement decreases survival rates by approximately $50 \%$.

\section{Workup and Staging}

The evaluation of the patient to determine tumor stage is similar for glottic and supraglottic primaries. In both sites, the algorithms now explicitly recommend CT scan with contrast and thin cuts through the larynx, or MRI of the primary and neck; chest imaging is also recommended. These imaging tests are considered particularly important for accurate staging. Examination under anesthesia with endoscopy is also recommended. A barium esophagram is recommended for patients with subglottic tumors. Speech, swallowing, and dental evaluation should be done if indicated. Multidisciplinary consultation is particularly important for both sites because of the potential for loss of speech and, in some instances, for swallowing dysfunction.

\section{Treatment}

The treatment of patients with laryngeal cancer is divided into 3 categories: (1) tumors of the glottic larynx, (2) tumors of the supraglottic larynx without positive nodes (N0), and (3) tumors of the supraglottic larynx with positive nodes $(\mathrm{N}+)$.

For patients with carcinoma in situ of the larynx, recommended treatment options include endoscopic removal (stripping, laser) or RT. NCCN also encourages participation in clinical trials. For invasive cancer, surgery (partial laryngectomy through either endoscopic or open approaches) and radiotherapy are equally effective for early-stage glottic or supraglottic cancers. The choice of treatment modality depends on functional outcome, the patient's wishes, reliability of follow-up, and general medical condition.

Management of the neck is dictated by the risk of occult nodal spread. Participation in clinical trials is preferred for patients with locally advanced laryngeal cancer requiring total laryngectomy. Resectable, advanced-stage supraglottic and glottic primaries can be managed surgically with a combined modality approach consisting of either (1) total laryngectomy, or (2) concurrent systemic therapy/RT cisplatin (category 1) preferred. ${ }^{115}$ In patients with laryngeal cancer, radiotherapy with concurrent administration of cisplatin is superior either to induction chemotherapy followed by radiotherapy or to radiotherapy alone for laryngeal preservation and locoregional control. ${ }^{115}$ Selected cases can be managed with conservation surgical techniques that preserve vocal function.

Patients with locally advanced disease who desire laryngeal preservation should receive treatment with concurrent chemotherapy consisting of cisplatin $100 \mathrm{mg} / \mathrm{m}^{2}$ on days 1, 22, and 43 (category 1) preferred and radiotherapy; ${ }^{115}$ the second option is definitive RT (without chemotherapy) for patients who are medically unfit or refuse 
chemotherapy. Surgery is reserved for managing the neck as indicated, for those patients whose disease persists after radiotherapy, or those patients who develop a subsequent locoregional recurrence.

The NCCN recommendations for managing locally advanced, resectable glottic and supraglottic cancers requiring laryngectomy reflect the results of Intergroup trial R91-11. ${ }^{115}$ Before 2002, either induction chemotherapy with cisplatin/5-FU followed by radiotherapy or definitive radiotherapy alone (without chemotherapy) were the standard of care options recommended in the NCCN H\&N guidelines based on the results of the Veterans Administration (VA) Laryngeal Cancer Study Group trial published in $1991 .{ }^{116}$ In the 2002-2008 versions of the guidelines, concurrent radiotherapy and cisplatin $100 \mathrm{mg} / \mathrm{m}^{2}$ is the recommended option for achieving laryngeal preservation. R91-11 was a successor trial to the Veterans Administration trial and compared 3 non-surgical regimens: (1) induction cisplatin/5-FU followed by RT (control arm and identical to that in the VA trial); (2) concurrent RT and cisplatin $100 \mathrm{mg} / \mathrm{m}^{2}$ days 1,22 , and 43; and (3) RT alone.

Radiotherapy was uniform in all 3 arms, 70 Gy/7 wks, 2 Gy/fx. Laryngectomy was used for salvage of treatment failures in all arms. Stage III and IV (M0) patients were eligible, excluding T1 primaries and high-volume T4 primaries (tumor extending more than $1 \mathrm{~cm}$ into the base of tongue or tumor penetrating through cartilage). The key findings of the trial were a statistically significant higher 2-year laryngeal preservation (local control) rate for concurrent RT with cisplatin of $88 \%$, compared to $74 \%$ with induction chemotherapy and to $69 \%$ with RT alone; no significant difference in laryngeal preservation between induction and RT alone treatments; and similar survival for all treatment groups. These R91-11 results changed the standard of care to concurrent RT and cisplatin (category 1 ) preferred for achieving laryngeal preservation for T3, NO and T4a, NO supraglottic cancers and for most T3, any $\mathrm{N}$ glottic cancers. ${ }^{115}$
For patients with glottic T4a tumors, the standard approach is a laryngectomy with ipsilateral thyroidectomy and neck dissection as indicated. For selected patients with glottic T4a tumors, the panel recommends 1) considering concurrent chemoradiation, or 2) clinical trials testing function-preserving surgical or nonsurgical approaches.

For managing $\mathrm{T} 4 \mathrm{a}$, NO supraglottic primaries, the panel made a distinction between (1) high-volume, base-of-tongue involvement ( $>1$ $\mathrm{cm}$ ) or tumor penetration through cartilage; and (2) low-volume disease with no cartilage penetration on imaging or with $1 \mathrm{~cm}$ or less extension into the base of the tongue. This later category of T4a supraglottic patients was eligible for Intergroup trial R91-11. The committee prefers nonsurgical, larynx-preserving treatment with concurrent RT and systemic therapy with cisplatin (category 1 ) for patients with low-volume disease whose tumor does not penetrate through cartilage. ${ }^{115}$ In contrast, the recommended options for those with high-volume T4a, N+ disease (for example, cartilage destruction, skin involvement, massive invasion of the base of the tongue) are either (1) laryngectomy, ipsilateral thyroidectomy with ipsilateral or bilateral neck dissection followed by chemotherapy/RT (category 1 for chemo/RT); or (2) a clinical trial (see SUPRA-8). Definitive radiotherapy alone (without chemotherapy) is reserved for patients in the poor medical risk category.

\section{Follow-up/Surveillance}

It is particularly important for nonsurgically treated patients to have careful and regular follow-up examinations by a trained H\&N surgical oncologist so that any local or regional recurrence is detected early, and salvage surgery (and neck dissection as indicated) is performed. Post-treatment baseline imaging (3-6 months) of the primary and neck is recommended for disease that is difficult to follow; repeat imaging if indicated by signs/symptoms on physical examination. If PET is used for follow-up, the first scan should not be performed sooner than 12 
weeks after treatment. Follow-up examinations in many of these patients need to be supplemented with serial endoscopy or highresolution, advanced radiologic imaging techniques because of the scarring, edema, and fibrosis that occur in the laryngeal tissues and neck after high-dose radiation. Speech, hearing, \& swallowing evaluation and rehabilitation may be useful, as indicated. Smoking cessation counseling is recommended.

\section{Carcinoma of the Nasopharynx}

Carcinoma of the nasopharynx is uncommon in the United States. Among $\mathrm{H} \& \mathrm{~N}$ cancers, it has the highest propensity to metastasize to distant sites. Nasopharyngeal cancer also poses a significant risk for isolated local recurrences after definitive radiation (without chemotherapy) for locally advanced disease. ${ }^{117-120}$ Oddly enough, regional recurrences are uncommon in this disease, occurring in only $10 \%$ to $19 \%$ of patients. ${ }^{120,121}$

The NCCN H\&N guidelines for the evaluation and management of carcinoma of the nasopharynx attempt to address risk for both local and distant disease. RT was the standard treatment for all stages of this disease, until the mid-1990s, when trial data showed improved survival for locally advanced tumors treated with concurrent RT and cisplatin. ${ }^{121}$

Stage is accepted as prognostically important. The prognostic significance of histology is still controversial. Several retrospective reviews indicated local control and survival appear to depend on histologic subtypes, ${ }^{120-124}$ whereas one study found no association between histology and these outcomes. ${ }^{125}$ The World Health Organization (WHO) classification for nasopharyngeal cancer is used most often. Type 1 represents well to moderately well-differentiated squamous cell cancers. Type 2 denotes nonkeratinizing tumors, including transitional carcinoma and lymphoepithelioma. Type 3 represents undifferentiated carcinomas, including lymphoepithelioma, anaplastic, clear cell, and spindle cell variants.

\section{Workup and Staging}

The workup of nasopharyngeal cancer includes a history, physical examination, nasopharyngeal examination and biopsy, dental evaluation if indicated, and appropriate diagnostic imaging studies (for example, MRI and/or CT with contrast). These studies are important to determine the full extent of tumor in order to assign stage appropriately and to design radiation ports that will encompass all the disease with appropriate doses. Chest imaging should also be obtained.

Multidisciplinary consultation is encouraged. The 2002 AJCC staging classification is used as the basis for treatment recommendations (see Table 2). For patients with WHO class 2-3/N2-3 disease, imaging for distant metastases (that is, chest, liver, bone) may include PET scan and/or CT.

\section{Treatment}

Treatment options are subdivided according to $\mathrm{T}, \mathrm{N}$, and $\mathrm{M}$ status, rather than by stage alone. Patients with early-stage nasopharyngeal tumors (T1, NO, MO, and T2a, NO, MO tumors) may be treated with definitive RT alone (without chemotherapy) to the nasopharynx, with elective radiation to the neck. For early-stage cancer, radiation doses of at least 70 Gy given with standard fractions are necessary for control of gross tumor (see NASO-A). The local control rate for these tumors ranges from $80 \%$ to $90 \%$, whereas T3-4 tumors have a control rate of $30 \%$ to $65 \%$. $^{122,126}$

The combination of RT and platinum-based chemotherapy has been shown to increase the local control rate from $54 \%$ to $78 \%$. The Intergroup trial 0099, which randomly assigned patients to chemotherapy plus external-beam RT versus external radiation alone, closed early when an interim analysis disclosed a significant survival 
and progression-free survival advantage favoring the combined chemotherapy and radiation group. ${ }^{127}$ The addition of chemotherapy also decreased local, regional, and distant recurrence rates. A similar randomized study conducted in Singapore, which was modeled after the Intergroup treatment regimen, continued to show the benefit of the addition of chemotherapy to radiation therapy. Adjuvant chemotherapy after combined chemotherapy and radiation was also given in this trial. ${ }^{128}$ In addition, the administration of the cisplatin dose was spread out over several days, and this regimen appeared to reduce toxicity while still providing a beneficial antitumor effect.

The guidelines recommend combined chemotherapy (cisplatin) plus radiotherapy for T1-T2a, N1-3; and for T2b-T4a, any N lesions (stages IIB, III, IVA, IVB). The scheduling and doses of chemotherapy are those used in the intergroup trial 0099. Although an unusual occurrence, a patient with residual disease in the neck and a complete response at the primary should undergo a neck dissection. Initial therapy for patients who present with metastatic disease (stage IV) should consist of a platinum-based combination chemotherapy regimen. If a complete response is achieved, definitive RT alone (without chemotherapy) should be administered to the primary tumor and neck area. For earlystage cancer, radiation doses of at least 70 Gy given with standard fractions are necessary for control of gross tumor. In patients with metastatic carcinoma who have failed platinum-based therapy, a tripletbased combination using paclitaxel, carboplatin, and gemcitabine may be useful. ${ }^{129}$ Likewise, cetuximab plus carboplatin may be useful for patients with recurrent or metastatic nasopharyngeal cancer who have failed platinum-based therapy. ${ }^{130}$ Gemcitabine monotherapy or in combination with cisplatin may also be useful. ${ }^{131-133}$

\section{Follow-up/Surveillance}

For patients whose nasopharyngeal cancer has been treated, the recommended follow-up includes periodic physical examination and an assessment of thyroid function (that is, the TSH level should be determined every 6 to 12 months). Increased TSH levels have been detected in $20 \%$ to $25 \%$ of patients who received neck irradiation. ${ }^{134}$ Post-treatment baseline imaging of the primary and neck is recommended; repeat imaging if indicated by signs/symptoms on physical examination. Speech, hearing, \& swallowing evaluation and rehabilitation and dental evaluation may be useful, as indicated. Smoking cessation counseling is recommended.

\section{Advanced Head and Neck Cancer}

Advanced H\&N cancer includes newly diagnosed but unresectable disease (see "Head and Neck Surgery"), recurrent disease, and metastatic disease. The treatment goal for patients with newly diagnosed but unresectable disease is cure. For the recurrent disease group, the goal is cure (if surgery or radiation remains feasible) or palliation (if the patient has received previous radiotherapy and the disease is unresectable). The goal for patients with metastatic disease is palliation or prolongation of life.

\section{Treatment}

Participation in clinical trials is preferred for all patients with advanced H\&N cancer. For patients with unresectable disease, such trials include testing altered fraction radiotherapy schedules, concurrent chemoradiotherapy, and novel radiosensitizers. For patients with recurrent disease not amenable to curative therapy and patients with metastatic disease, studies include trials of new agents and reirradiation.

\section{Unresectable Disease}

For patients with a performance status (PS) of 0 or 1 , the standard treatment of newly diagnosed, unresectable disease is concurrent cisplatin (single agent) or carboplatin-based chemotherapy and radiotherapy (category 1 ). ${ }^{135}$ The panel disagreed regarding whether 
induction chemotherapy (cisplatin) followed by chemoradiation should be used (category 3 ) for patients with a PS of 0 or 1 . For those with a PS of 2, the recommended treatment is generally radiotherapy with or without concurrent chemotherapy; again, the panel disagreed about using induction chemotherapy followed by RT (category 3 ). For those with PS of 3 , the recommended treatment is generally radiotherapy alone or, in some cases, best supportive care.

Many randomized trials ${ }^{135-144}$ and meta-analyses of clinical trials ${ }^{145-149}$ demonstrate significantly improved overall survival, disease-free survival, and local control when concomitant or alternating chemotherapy and radiation is compared with radiotherapy alone. All combined chemoradiotherapy regimens are associated with various degrees of enhanced mucosal toxicities, which require close patient monitoring, ideally provided by a team experienced in treating $\mathrm{H} \& \mathrm{~N}$ cancer patients. The various single-agent chemoradiotherapy regimens have not been directly compared in randomized trials. Therefore, no optimal standard regimen is defined. Single-agent cisplatin plus RT is effective and relatively easy to administer. ${ }^{135}$ In a phase III randomized trial, cetuximab-based chemoradiotherapy improved locoregional control and overall survival in patients with stage III/IV head and neck cancer; ${ }^{150}$ however, there have been concerns about the method used to assess locoregional control. ${ }^{151} \mathrm{~A}$ randomized phase II study in patients with advanced H\&N (oral cavity, oropharynx, or hypopharynx) suggested that cisplatin plus paclitaxel and RT may yield better overall survival than either cisplatin plus 5-FU and RT or hydroxyurea, 5-FU and RT, although statistical comparisons were not possible. ${ }^{152,153}$

Based on published data, concurrent chemoradiation typically uses conventional fractionation at $2.0 \mathrm{~g}$ per fraction to $70 \mathrm{~Gy}$ or more in 7 weeks with single-agent cisplatin given every 3 weeks at $100 \mathrm{mg} / \mathrm{m}^{2}$. Use of other fraction sizes (for example, 1.8 Gy, conventional), multiagent chemotherapy, or altered fractionation with chemotherapy has been evaluated, but there is no consensus on the optimal approach. In general, the use of concurrent chemoradiation carries a high toxicity burden, and altered fractionation or multiagent chemotherapy will likely further increase the toxicity burden. For any chemoradiation approach, close attention should be paid to published reports for the specific chemotherapy agent, dose, and schedule of administration. Chemoradiation should be performed by an experienced team and should include substantial supportive care.

Bonner and colleagues have randomly assigned 424 patients with locally advanced and measurable squamous cell carcinomas of the $\mathrm{H} \& \mathrm{~N}$ to receive definitive radiotherapy with or without cetuximab. ${ }^{150}$ Locoregional control and survival (49 months versus 29.3 months, $P=.03$ ) were significantly improved in patients treated with radiotherapy and cetuximab compared to radiotherapy alone. Radiotherapy and cetuximab may provide a therapeutic option for patients not considered medically fit for standard chemoradiotherapy regimens. However, more study is needed.

\section{Recurrent Disease}

Surgery is recommended for resectable recurrent locoregional disease, usually followed by radiation if it has not yet been administered. If the recurrence is unresectable and the patient did not have prior RT, then radiotherapy with concurrent cisplatin or carboplatin-based chemotherapy is recommended (category 1 ) for patients with PS of 0 or 1 (see ADV-1). For patients with recurrent disease not amenable to curative-intent radiation or surgery, the treatment approach is the same as that for patients with metastatic disease. For select patients, enrollment in a clinical trial of re-irradiation may be appropriate.

A study in patients with metastatic or recurrent $\mathrm{H} \& \mathrm{~N}$ cancer found no significant difference in survival when comparing cisplatin plus 5-FU with cisplatin plus paclitaxel. ${ }^{154}$ Other combination regimens used in this patient population include carboplatin plus $5-\mathrm{FU}^{155}$ and cetuximab plus 
cisplatin. ${ }^{156,157}$ These regimens are all listed as treatment options for this patient population.

Squamous cell carcinomas emerge after the accumulation of multiple genomic events. In a multistep process, there appear to be essential molecular alterations, which confer a survival advantage for cancer cells. The epidermal growth factor receptor (EGFR) is a

transmembrane glycoprotein, activation of which triggers a cascade of downstream intracellular signaling events important for regulation of epithelial cell growth. Overexpression of EGFR and/or common ligands has been observed in greater than $90 \%$ of squamous cell carcinomas of the H\&N. This finding has led to the development of EGFR inhibitors, such as the monoclonal antibody cetuximab and small molecule tyrosine kinase inhibitors (such as erlotinib and gefitinib).

In phase II trials, cetuximab was combined with cisplatin in treating patients with recurrent disease that was refractory to platinum-based chemotherapy. ${ }^{158-160}$ Objective tumor response was observed in $12 \%$ to $14 \%$ of patients. Vermorken and colleagues reported response in $13 \%$ of platinum refractory patients, treated with cetuximab administered as a single agent. ${ }^{161}$ Burtness and colleagues ${ }^{156}$ did a direct comparison of cisplatin plus cetuximab versus cisplatin plus placebo as first-line treatment of recurrent disease; they reported a significant improvement in response rate (26\% versus $10 \%$, respectively). Taken together, this data indicates that cetuximab does not overcome platinum resistance, the single-agent response rate is on the order of $12 \%$ to $14 \%$. In combination with cisplatin as first-line treatment, a synergistic effect is observed. Moreover, the preliminary results of a phase III trial (Extreme) of 442 patients with recurrent or metastatic squamous cell carcinoma found that cetuximab plus cisplatin/5-FU or carboplatin/ 5-FU improved median survival when compared to the standard chemotherapy doublet (10.1 months versus 7.4 months, $P=.036){ }^{162}$
Other recent phase II trials using either cisplatin/docetaxel/erlotinib or paclitaxel/cetuximab also suggest benefit from EGFR inhibition. ${ }^{163,164}$

\section{Metastatic Disease}

Palliative adjunctive measures include radiotherapy to areas of symptomatic disease, analgesics, and investigational agents aimed at controlling locally advanced tumors. Single agents and combination systemic chemotherapy regimens are both used. Response rates to single agents range from $15 \%$ to $35 \%$. The most active agents include cisplatin, carboplatin, paclitaxel, docetaxel, 5-FU, methotrexate, ifosfamide, bleomycin, gemcitabine (for nasopharyngeal cancer), and cetuximab. The most active regimens include (1) cisplatin or carboplatin, plus 5-FU; ${ }^{154,155}$ or (2) cisplatin or carboplatin, plus a taxane. ${ }^{154}$ These regimens result in higher response rates of $30 \%$ to $40 \%$.

Randomized trials assessing a combination of cisplatin plus 5-FU versus single-agent therapy with cisplatin, $5-\mathrm{FU}$, or methotrexate have demonstrated significantly higher response rates for the combination regimen. No difference in overall survival, however, is demonstrable. ${ }^{154,155,165-167}$ The median survival with chemotherapy is approximately 6 months, and the 1-year survival rate is approximately $20 \%$. Achievement of a complete response is associated with longer survival and, although infrequent, has been reported more often with combination regimens.

The standard treatment of patients with incurable, recurrent, or metastatic H\&N cancer should be dictated, in large part, by the patient's PS (see ADV-2). Individuals with a good PS (0-1) may be offered combination or single-agent chemotherapy. Patients should be fully informed about the goals of treatment, cost of combination chemotherapy, and potential for added toxicity. For patients with a PS of 2, single-agent chemotherapy or best supportive care is most appropriate. For patients with a good PS who relapse after first-line 
chemotherapy, second-line treatment in a clinical trial or best supportive care is appropriate. For patients with a PS of 3 , best supportive care is appropriate.

\section{Disclosures for the NCCN Head and Neck Cancers Guidelines Panel}

At the beginning of each panel meeting to develop NCCN guidelines, panel members disclosed the names of companies, foundations, and/or funding agencies from which they received research support; for which they participate in speakers' bureau, advisory boards; and/or in which they have equity interest or patents. Members of the panel indicated that they have received support from the following: Amgen Inc; AstraZeneca; Boehringer Ingelheim, Bristol Myers-Squibb; Genentech Inc; GlaxoSmithKline; ImClone Systems Inc; Ingenium Excellentia, Inc. IRX Therapeutics; Novartis; Pfizer Inc; and sanofi-aventis. Some panel members do not accept any support from industry. The panel did not regard any potential conflicts of interest as sufficient reason to disallow participation in panel deliberations by any member. 


\section{Figure 1}

Anatomic sites and subsites of the head and neck

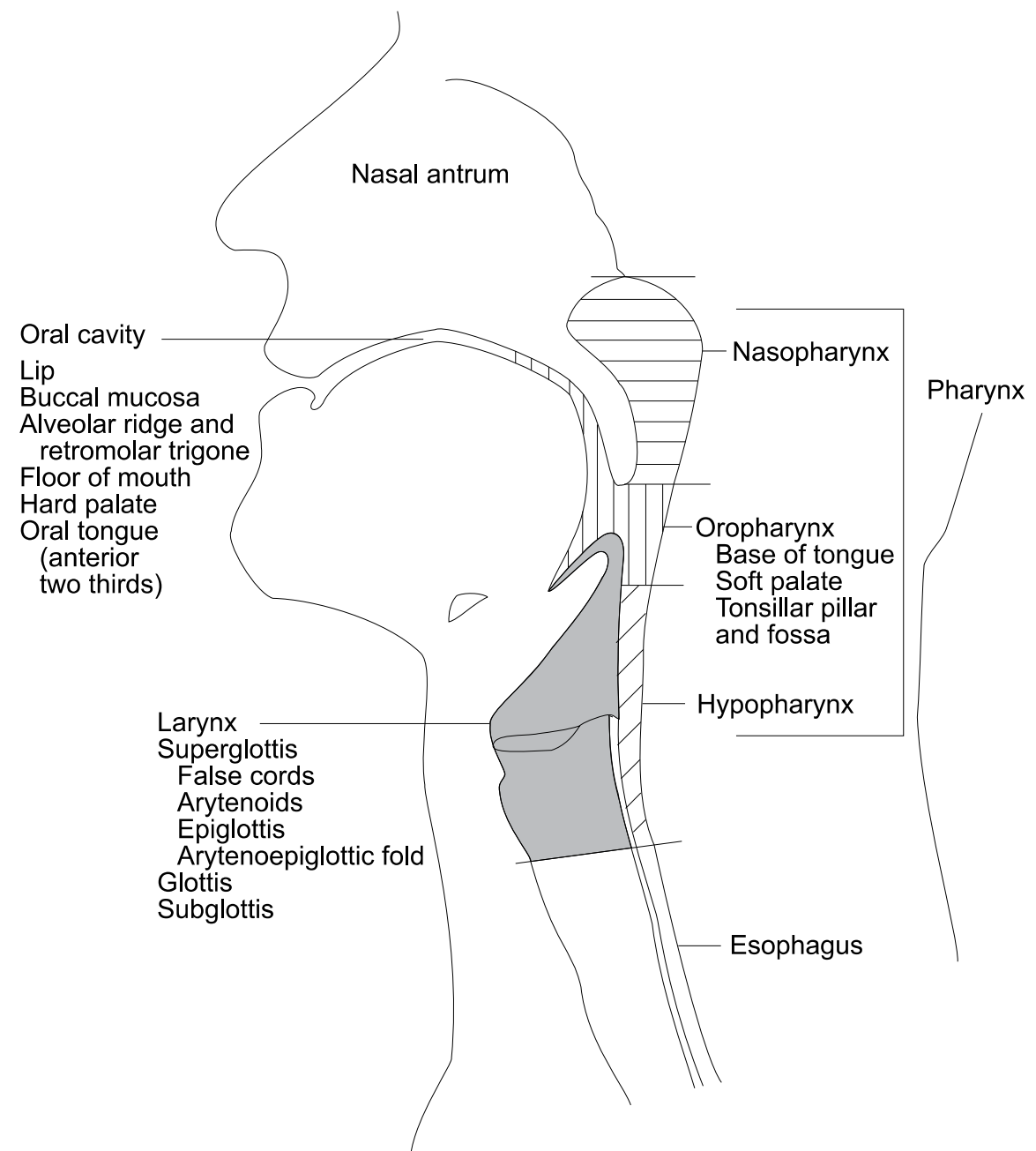

Reprinted with permission, from CMP Healthcare Media. Source: Cancer Management: A Multidisciplinary Approach, 9th ed. Pazdur R, Coia L, Hoskins W, et al (eds), Chapter 4. Copyright 2005, All rights reserved.

\section{Figure 2}

Level designation for cervical lymphatics in the right neck

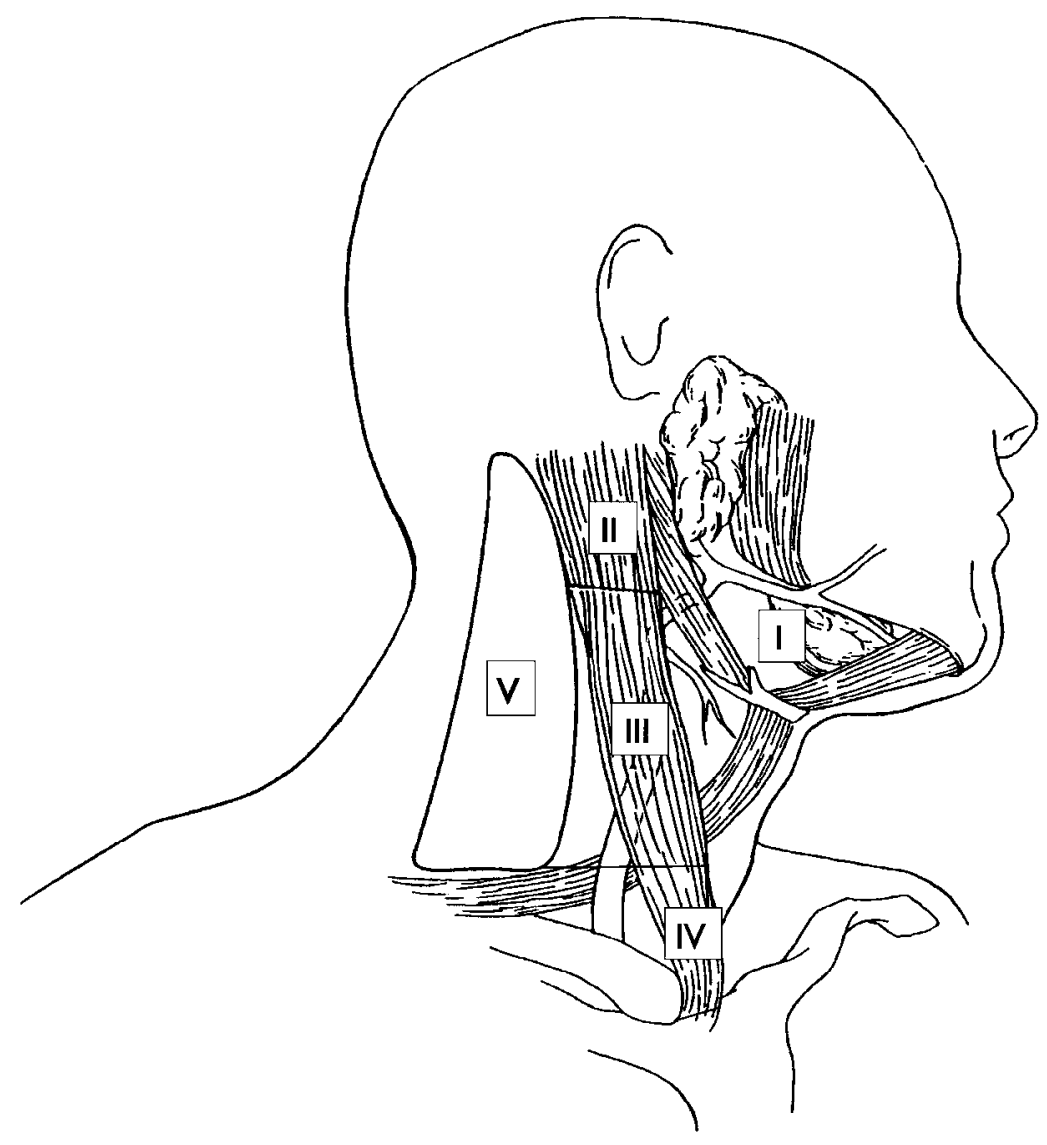

Reprinted with permission, from CMP Healthcare Media. Source: Cancer Management: A Multidisciplinary Approach, 9th ed. Pazdur R, Coia L, Hoskins W, et al (eds), Chapter 4. Copyright 2005, All rights reserved. 


\section{References}

1. Jemal A, Siegel R, Ward E, et al. Cancer Statistics, 2008. CA Cancer J Clin 2008;58:71-96.

2. Gillison ML, Koch WM, Capone RB, et al. Evidence for a causal association between human papillomavirus and a subset of head and neck cancers. J Natl Cancer Inst 2000;92(9):709-720.

3. Kreimer AR, Clifford GM, Boyle P, Franceschi S. Human papillomavirus types in head and neck squamous cell carcinomas worldwide: a systematic review. Cancer Epidemiol Biomarkers Prev 2005;14(2):467-475.

4. Applebaum KM, Furniss CS, Zeka A, et al. Lack of association of alcohol and tobacco with HPV16-associated head and neck cancer. J Natl Cancer Inst 2007;99(23):1801-1810.

5. D'Souza G, Kreimer AR, Viscidi R, et al. Case-control study of human papillomavirus and oropharyngeal cancer. N Engl J Med 2007;356(19):1944-1956.

6. Schlecht NF, Burk RD, Adrien L, et al. Gene expression profiles in HPV-infected head and neck cancer. J Pathol 2007;213(3):283-293.

7. Sturgis EM. Trends in head and neck cancer incidence in relation to smoking prevalence. Cancer 2007;110(7):1429-1435.

8. Greene FL, Page DL, Fleming ID, et al (eds). AJCC Cancer Staging Manual, Sixth Edition Springer-Verlag: New York 2002.

9. Colasanto JM, Prasad P, Nash MA, et al. Nutritional support of patients undergoing radiation therapy for head and neck cancer.

Oncology 2005;19:371-382.

10. Schnoll RA, Zhang B, Rue M, et al. Brief physician-initiated quitsmoking strategies for clinical oncology settings: a trial coordinated by the Eastern Cooperative Oncology Group. J Clin Oncol 2003;21:355-365.
11. Gritz ER, Carr CR, Rapkin D, et al. Predictors of long-term smoking cessation in head and neck cancer patients. Cancer Epidemiol Biomarkers Prev 1993;2:261-270.

12. Feinstein AR. The pre-therapeutic classification of comorbidity in chronic disease. J Chron Dis 1970;23:455-469.

13. Charlson ME, Pompei $P$, Ales $K L$, et al. A new method of classifying prognostic comorbidity in longitudinal studies: development and validation. J Chronic Dis 1987;40:373-383.

14. Piccirillo JF. Importance of comorbidity in head and neck cancer. Laryngoscope 2000;110:593-602.

15. Piccirillo JF, Lacy PD, Basu A, et al. Development of a new head and neck cancer-specific comorbidity index. Arch Otolaryngol Head Neck Surg 2002;128:1172-1179.

16. Piccirillo JF. Impact of comorbidity and symptoms on the prognosis of patients with oral carcinoma. Arch Otolaryngol Head Neck Surg 2000;126:1086-1088.

17. Chen AY, Matson LK, Roberts D, et al. The significance of comorbidity in advanced laryngeal cancer. Head Neck 2001;23:566-572.

18. Singh B, Bhaya M, Stern J, et al. Validation of the Charlson comorbidity index in patients with head and neck cancer: a multiinstitutional study. Laryngoscope 1997;107:1469-1475.

19. Hall SF, Rochon PA, Streiner DL, et al. Measuring comorbidity in patients with head and neck cancer. Laryngoscope 2002;112:1988-1996.

20. Hall SF, Groome PA, Rothwell D. The impact of comorbidity on the survival of patients with squamous cell carcinoma of the head and neck. Head Neck 2000;22:317-322.

21. Ribeiro KC, Kowalski LP, Latorre MR. Impact of comorbidity, symptoms, and patients' characteristics on the prognosis of oral carcinomas. Arch Otolaryngol Head Neck Surg 2000;126:1079-1085. 
22. de Graeff A, de Leeuw JR, Ros WJ, et al. Pretreatment factors predicting quality of life after treatment for head and neck cancer. Head Neck 2000;22:398-407.

23. Funk GF, Karnell LH, Whitehead S, et al. Free tissue transfer versus pedicled flap cost in head and neck cancer. Otolaryngol Head Neck Surg 2002;127:205-212.

24. Farwell DG, Reilly DF, Weymuller EA Jr., et al. Predictors of perioperative complications in head and neck patients. Arch Otolaryngol Head Neck Surg 2002;128:505-511.

25. Kaplan MH, Feinstein AR. The importance of classifying initial comorbidity in evaluating the outcome of diabetes mellitus. J Chronic Dis 1974;27:387-404.

26. Bang D, Piccirillo JF, Littenberg B, et al. The Adult Comorbidity Evaluation-27 (ACE-27) test: a new comorbidity index for patients with cancer. Paper presented at: Annual Meeting of the American Society of Clinical Oncology; May 20, 2000, 2000; New Orleans, La.

27. Piccirillo JF, Costas I, Claybour $P$, et al. The measurement of comorbidity by cancer registries. J Reg Management 2003;30:8-14.

28. Patrick DL, Erickson P. Health status and health policy: quality of life in health care evaluation and resource allocation. New York: Oxford University Press; 1993.

29. Yueh B. Measuring and Reporting Quality of Life in Head and Neck Cancer, 2002; McLean, Virginia.

30. Rogers SN, Gwanne S, Lowe D, et al. The addition of mood and anxiety domains to the University of Washington quality of life scale. Head Neck 2002;24:521-529.

31. Bjordal K, Hammerlid E, Ahlner-Elmqvist M, et al. Quality of life in head and neck cancer patients: validation of the European Organization for Research and Treatment of Cancer Quality of Life QuestionnaireH\&amp;N35. J Clin Oncol 1999;17:1008-1019.
32. Cella DF. Manual for the Functional Assessment of Cancer Therapy (FACT) Measurement System (version 3). Chicago: Rush Medical Center; 1994.

33. List MA, D'Antonio LL, Cella DF, et al. The Performance Status Scale for Head and Neck Cancer Patients and the Functional Assessment of Cancer Therapy-Head and Neck Scale. A study of utility and validity. Cancer 1996;77:2294-2301.

34. Byers RM. Neck dissection: Concepts, controversies, and technique. Semin Surg Oncol 1991;7:9-13.

35. Stringer SP. Current concepts in surgical management of neck metastases for head and neck cancer. Oncology 1995;9:547-554.

36. Candela FC, Kothari K, Shah JP. Patterns of cervical node metastases from squamous cell carcinoma of the oropharynx and hypopharynx. Head Neck 199012:197-203.

37. Candela FC, Shah L, Jaques DP, et al. Patterns of cervical node metastases from squamous cell carcinoma of the larynx. Arch Otolaryngol Head Neck Surg 199016:432-435.

38. Shah JP, Candela FC, Poddar AK. The patterns of cervical lymph node metastases from squamous cell carcinomas of the oral cavity. Cancer 1990;66:109-113.

39. Shah JP, Cendon RA, Farr HW, et al. Carcinoma of the oral cavity: Factors affecting treatment failure at the primary site and neck. Am J Surg 1976;132:504-507.

40. Looser KG, Shah JP, Strong EW. The significance of "positive margins" in surgically resected epidermoid carcinomas. Head Neck Surg 1978;1:107-111.

41. Johnson JT, Barnes EL, Myers EN, et al. The extracapsular spread of tumors in cervical node metastasis. Arch Otolaryngol Head Neck Surg 1981;107:725-729.

42. Feldman $\mathrm{M}$, Fletcher $\mathrm{FH}$. Analysis of the parameters relating to failures above the clavicle in patients treated by postoperative 
irradiation for squamous-cell carcinomas of the oral cavity or oropharynx. J Radiat Oncol Biol Phys 1982;8:27-30.

43. Mirimanoff RO, Wang CC, Doppke KP. Combined surgery and postoperative radiation therapy for advanced laryngeal and hyopharyngeal carcinomas. Int J Radiat Oncol Biol Phys 1985;11:499-504.

44. Peters LJ, Goepfert H, Ang KK, et al. Evaluation of the dose for postoperative radiation therapy of head and neck cancer: First report of a prospective randomized trial. Int J Radiat Oncol Biol Phys 1993;26:3-9.

45. Bernier J, Domenge C, Ozsahin M, et al. Postoperative irradiation with or without concomitant chemotherapy for locally advanced head and neck cancer. N Engl J Med 2004;350:1945-1952.

46. Cooper JS, Pajak TF, Forastiere AA, et al. Postoperative concurrent radiotherapy and chemotherapy for high-risk squamous-cell carcinoma of the head and neck. N Engl J Med 2004;350:1937-1944.

47. Bernier J, Cooper JS, Pajak TF, et al. Defining risk levels in locally advanced head and neck cancers: A comparative analysis of concurrent postoperative radiation plus chemotherapy trials of the EORTC (\#22931) and RTOG (\# 9501). Head Neck 2005;27:843-850.

48. Bachaud JM, Cohen-Jonathan E, Alzieu C, et al. Combined postoperative radiotherapy and weekly cisplatin infusion for locally advanced head and neck carcinoma: final report of a randomized trial. Int J Radiat Oncol Biol Phys 1996;36(5):999-1004.

49. Jeremic B, Milicic B, Dagovic A, et al. Radiation therapy with or without concurrent low-dose daily chemotherapy in locally advanced, nonmetastatic squamous cell carcinoma of the head and neck. Clin Oncol 2004;22(17):3540-3548.

50. Harwood AR, Beale FA, Cummings BJ, et al. T2 Glottic cancer: An analysis of dose-time volume factors. Int J Radiat Oncol Biol Phys 1981;7:1501-1505.
51. Amornmarn R, Prempreet T, Viravathana T, et al. A therapeutic approach to early vocal-cord carcinoma. Acta Radiol Oncol 1985;24:321-325.

52. Schwaibold F, Scariato A, Nunno M, et al. The effect of fraction size on control of early glottic cancer. Int J Radiat Oncol Biol Phys 1988;14:451-454.

53. Kim RY, Marks ME, Salter MM. Early-stage glottic cancer: Importance of dose fractionation in radiation therapy. Radiology 1992;182:273-275.

54. Parson JT. Time-dose-volume relationships in radiation therapy. In: Million RR, Cassisi NJ, eds. Management of Head and Neck Cancer: A Multidisciplinary Approach, 2nd ed. Philadelphia: JB Lippincott 1994;203-243.

55. Thames HD, Withers HR, Peters LJ, et al. Changes in early and late radiation responses with altered dose fractionation: Implications for dose-survival relationships. Int J Radiat Oncol Biol Phys 1982;8:219-226.

56. Withers HR, Thames HD, Peters LJ. Differences in the fractionation response of acutely and late-responding tissues. In: Kaercher $\mathrm{KH}$, Kogelnik HD, Reinartz G, eds. Progress in Radio-Oncology, vol 11 New York: Raven Press 1982;287-296.

57. Bourhis J, Wibault P, Lusinchi A, et al. Status of accelerated fractionation radiotherapy in head and neck squamous cell carcinomas. Curr Opin Oncol 1997;9:262-266.

58. Horiot JC, Le Fur R, N'Guyen T, et al. Hyperfractionation versus conventional fractionation in oropharyngeal carcinoma: Final analysis of a randomized trial of the EORTC cooperative group of radiotherapy. Radiother Oncol 1992;25:231-241.

59. Horiot JC. [Controlled clinical trials of hyperfractionated and accelerated radiotherapy in otorhinolaryngologic cancers] [Article in French]. Bull Acad Natl Med 1998;182:1247-1260; discussion 1261.]. 
60. Horiot JC, Bontemps P, Lagarde C. Accelerated fractionation (AF) compared to conventional fractionation (CF) improves loco-regional control in the radiotherapy of advanced head and neck cancers: Results of EORTC 22851 trial. Radiother Oncol 1997;44:111-121.

61. Fu KK, Pajak TF, Trotti A, et al. A radiation therapy oncology group (RTOG) phase III randomized study to compare hyperfractionation and two variants of accelerated fractionation to standard fractionation radiotherapy for head and neck squamous cell carcinomas: first report of RTOG 9003. Int J Radiation Oncol Biol Phys 2000;48:7-16.

62. Trotti A, Fu K., Pajak T, et al. Long term outcomes of RTOG 90-03: A comparison of hyperfractionation and two variants of accelerated fractionation to standard fractionation radiotherapy for head and neck squamous cell carcinoma [Abstract \#116]. Proc Amer Soc Thera Rad Onc (ASTRO), Denver, CO. Int J Radiat Oncol Biol Phys 2005;63:S70-S71.

(www.oncolink.com/conferences/article.cfm?c=3\&s=33\&ss=197\&id=1290)

63. Bourhis J, Overgaard J, Audry H, et al; Meta-Analysis of Radiotherapy in Carcinomas of Head and neck (MARCH) Collaborative Group. Hyperfractionated or accelerated radiotherapy in head and neck cancer: a meta-analysis. Lancet 2006;368(9538):843-854.

64. Budach V, Stuschke M, Budach W, et al. Hyperfractionated accelerated chemoradiation with concurrent fluorouracil-mitomycin is more effective than dose-escalated hyperfractionated accelerated radiation therapy alone in locally advanced head and neck cancer: final results of the radiotherapy cooperative clinical trials group of the German Cancer Society 95-06 Prospective Randomized Trial. Clin Oncol 2005;23(6):1125-1135.

65. Budach W, Hehr T, Budach V, et al. A meta-analysis of hyperfractionated and accelerated radiotherapy and combined chemotherapy and radiotherapy regimens in unresected locally advanced squamous cell carcinoma of the head and neck. BMC Cancer 2006 Jan 31;6:28.
66. Fu KK, Chan EK, Phillips TL, et al. Time, dose, and volume factors in interstitial radium implant of carcinoma of the oral tongue. Radiology 1976;119:209-213.

67. Pigneux J, Richard PM, Lagarde C. The place of interstitial therapy using iridium 192 in the management of carcinoma of the lip. Cancer 1979;43:1073-1077.

68. Mendenhall WM, Van Cise WS, Bova FJ, et al. Analysis of timedose factors in squamous cell carcinoma of the oral tongue and floor of mouth treated with radiation therapy alone. Int J Radiat Oncol Biol Phys 1981;7:1005-1011.

69. Puthawala AA, Syed AM, Neblett D, et al. The role of afterloading iridium 192 implant in the management of carcinoma of the tongue. Int J Radiat Oncol Biol Phys 1981;7:407-412.

70. Goffinet DR, Fee WE, Wells J, et al. Iridium-192 pharyngoepiglottic fold interstitial implants: The key to successful treatment of base tongue carcinoma by radiation therapy. Cancer 1985;55:941-948.

71. Puthawala AA, Syed AM, Eads DL, et al. Limited external irradiation and interstitial iridium-192 implant in the treatment of squamous cell carcinoma of the tonsillar region. Int J Radiat Oncol Biol Phys 1985;11:1595-1602.

72. Vikram B, Strong E, Shah JP, et al. A non-looping afterloading technique for base of tongue implants: Results of the first 20 patients. Int J Radiat Oncol Biol Phys 1985;11:1853-1855.

73. Crook J, Mazeron JJ, Marinello G, et al. Combined external irradiation and interstitial implantation for T1 and T2 epidermoid carcinomas of base of tongue: The Creteil experience (1971-1981). Int J Radiat Oncol Biol Phys 1988;15:105-114.

74. Mazeron JJ, Belkacemi Y, Simon JM, et al. Place of iridium 192 implantation in definitive irradiation of faucial arch squamous cell carcinomas. Int J Radiat Oncol Biol Phys 1993;27:251-257.

75. Butler EB, Teh BS, Grant WH, et al. Smart (simultaneous modulated accelerated radiation therapy) boost: a new accelerated 
fractionation schedule for the treatment of head and neck cancer with intensity modulated radiotherapy. Int J Radiat Oncol Biol Phys 1999;45:21-32.

76. Eisbruch A, Ten Haken RK, Kim HM, et al. Dose, volume, and function relationships in parotid salivary glands following conformal and Intensity-Modulated irradiation of head and neck cancer. Int J Radiat Oncol Biol Phys 1999;45:577-587.

77. Dawson LA, Anzai Y, Marsh L, et al. Patterns of local-regional recurrence following parotid-sparing conformal and segmental IntensityModulated Radiotherapy for Head and Neck Cancer. Int J Radiat Oncol Biol Phys 2000;46:1117-1126.

78. Chao KSC, Low DA, Perez CA, et al. Intensity modulated radiation therapy for head and neck cancers: the Mallinckrodt experience. Int J Cancer (Radiat Oncol Invest) 2000;90:92-103.

79. Mittal BB, Kepka A, Mahadevan A, et al. Tissue/Dose Compensation to reduce toxicity from combined radiation and chemotherapy for advanced Head and Neck Cancers. Int J Cancer (Radiat Oncol Invest) 2001;96:61-70.

80. Teh BS, Mai WY, Grant WH 3rd, et al. Intensity modulated radiotherapy (IMRT) decreases treatment-related morbidity and potentially enhances tumor control. Cancer Invest 2002;20:437-451.

81. Chen YJ, Kuo JV, Ramsinghani NS, et al. Intensity-modulated radiotherapy for previously irradiated, recurrent head-and-neck cancer. Med Dosim 2002;27:171-176.

82. De Neve W, Duthoy W, Boterberg T, et al. Intensity Modulated Radiation Therapy: Results in Head and Neck cancer and Improvements ahead of us. Int J Radiat Oncol Biol Phys 2003;55:460.

83. Sanguineti G, Endres EJ, Gunn BG, Brent Parker B. Is there a "mucosa-sparing" benefit of IMRT for head-and-neck cancer? Int J Radiat Oncol Biol Phys 2006;66:931-938.
84. Lee NY, Harris J, Garden A, et al. Phase II multi-institutional study of IMRT \pm chemotherapy for nasopharyngeal carcinoma (RTOG 0225): Preliminary results. Int J Radiat Oncol Biol Phys 2007;69:S13-S14.

85. Lee N, Xia P, Fischbein NJ, et al. Intensity-modulated radiation therapy for head-and-neck cancer: the UCSF experience focusing on target volume delineation. Int J Radiat Oncol Biol Phys 2003;57(1):49-60.

86. Dabaja B, Salehpour MR, Rosen I, et al. Intensity-modulated radiation therapy (IMRT) of cancers of the head and neck: comparison of split-field and whole-field techniques. Int J Radiat Oncol Biol Phys 2005;63(4):1000-1005. Epub 2005 Jun 22.

87. Lee N, Mechalakos J, Puri DR, Hunt M. Choosing an intensitymodulated radiation therapy technique in the treatment of head-andneck cancer. Int J Radiat Oncol Biol Phys 2007;68(5):1299-1309. Epub 2007 Jan 22.

88. Das IJ , Cheng C-W, Chopra KL, et al. Intensity-modulated radiation therapy dose prescription, recording, and delivery: patterns of variability among institutions and treatment planning systems. J Natl Cancer Inst 2008;100:300-307. Advance Access published on March 5, 2008.

89. Cohen ZR, Marmor E, Fuller GN, DeMonte F. Misdiagnosis of olfactory neuroblastoma. Neurosurg Focus 2002;12(5):e3.

90. Ejaz A, Wenig BM. Sinonasal undifferentiated carcinoma: clinical and pathologic features and a discussion on classification, cellular differentiation, and differential diagnosis. Adv Anat Pathol 2005;12(3):134-143.

91. Iezzoni JC, Mills SE. "Undifferentiated" small round cell tumors of the sinonasal tract: differential diagnosis update. Am J Clin Pathol 2005;124 Suppl:S110-S121.

92. Mendenhall WM, Mendenhall CM, Riggs CE Jr, et al. Sinonasal undifferentiated carcinoma. Am J Clin Oncol 2006;29(1):27-31. 
93. Kim BS, Vongtama R, Juillard G. Sinonasal undifferentiated carcinoma: case series and literature review. Am J Otolaryngol 2004;25(3):162-166.

94. Smith SR, Som P, Fahmy A, et al. A clinicopathological study of sinonasal neuroendocrine carcinoma and sinonasal undifferentiated carcinoma. Laryngoscope 2000;110(10 Pt 1):1617-1622.

95. Diaz EM Jr, Johnigan RH 3rd, Pero C, et al. Olfactory neuroblastoma: the 22-year experience at one comprehensive cancer center. Head Neck 2005;27(2):138-149.

96. McLean JN, Nunley SR, Klass C, et al. Combined modality therapy of esthesioneuroblastoma. Otolaryngol Head Neck Surg 2007;136(6):998-1002.

97. Bron LP, Traynor SJ, McNeil EB, O'Brien CJ. Primary and metastatic cancer of the parotid: comparison of clinical behavior in 232 cases. Laryngoscope 2003;113(6):1070-1075.

98. Furniss CS, McClean MD, Smith JF, et al. Human papillomavirus 16 and head and neck squamous cell carcinoma. Int J Cancer 2007;120(11):2386-2392.

99. Fakhry C, Gillison ML. Clinical implications of human papillomavirus in head and neck cancers. J Clin Oncol 2006;24(17):2606-2611.

100. Fakhry C, Westra WH, Li S, et al. Improved survival of patients with human papillomavirus-positive head and neck squamous cell carcinoma in a prospective clinical trial. J Natl Cancer Inst 2008;100(4):261-269. Epub 2008 Feb 12.

101. Denis F, Garaud P, Bardet E, et al. Final results of the 94-01 French Head and Neck Oncology and Radiotherapy Group randomized trial comparing radiotherapy alone with concomitant radiochemotherapy in advanced-stage oropharynx carcinoma. J Clin Oncol 2004;22:69-76. Epub 2003 Dec 02.

102. Vokes EE, Stenson K, Rosen FR, et al. Weekly carboplatin and paclitaxel followed by concomitant paclitaxel, fluorouracil, and hydroxyurea chemoradiotherapy: curative and organ-preserving therapy for advanced head and neck cancer. J Clin Oncol 2003;21:320-326.

103. Hitt R, Grau J, Lopez-Pousa A, et al. Phase II/III trial of induction chemotherapy (ICT) with cisplatin/5-fluorouracil (PF) vs. docetaxel (T) plus PF (TPF) followed by chemoradiotherapy (CRT) vs. CRT for unresectable locally advanced head and neck cancer (LAHNC) (abstract). ASCO Annual Meeting Proceedings (post-meeting edition). J Clin Oncol 2005;23:5578.

104. Hitt R, López-Pousa A, Martínez-Trufero J, et al. Phase III study comparing cisplatin plus fluorouracil to paclitaxel, cisplatin, and fluorouracil induction chemotherapy followed by chemoradiotherapy in locally advanced head and neck cancer. J Clin Oncol 2005;23(34):8636-8645. Epub 2005 Nov 7.

105. Hitt R, Grau J, Lopez-Pousa A, et al. Randomized phase II/III clinical trial of induction chemotherapy (ICT) with either cisplatin/5fluorouracil (PF) or docetaxel/cisplatin/5-fluorouracil (TPF) followed by chemoradiotherapy (CRT) vs. crt alone for patients (pts) with unresectable locally advanced head and neck cancer (LAHNC). ASCO Annual Meeting Proceedings (post-meeting edition). J Clin Oncol 2006;24:5515.

106. Posner MR, Hershock DM, Blajman CR, et al. Cisplatin and fluorouracil alone or with docetaxel in head and neck cancer. $N$ Engl J Med 2007;357(17):1705-1715.

107. Vermorken JB, Remenar E, van Herpen C, et al; EORTC 24971/TAX 323 Study Group. Cisplatin, fluorouracil, and docetaxel in unresectable head and neck cancer. N Engl J Med 2007;357(17):1695-1704.

108. Pinto LJ, Canary PCV, Araujo CMM, et al. Prospective randomized trial comparing hyperfractionated versus conventional radiotherapy in stages III and IV oropharyngeal carcinoma. Int J Radiat Oncol Biol Phys 1990;21:557-562. 
109. Kotwall C, Sako K, Razack MS, et al. Metastatic patterns in squamous cell cancer of the head and neck. Am J Surg 1987;154:439-442.

110. Lefebvre J-L, Chevalier D, Luboinski B, et al. Larynx preservation in pyriform sinus cancer: Preliminary results of a European

Organization for Research and Treatment of Cancer phase III trial.

J Natl Cancer Inst 1996;88:890-899.

111. Schrijvers D, Van Herpen C, Kerger J, et al. Docetaxel, cisplatin and 5-fluorouracil in patients with locally advanced unresectable head and neck cancer: a phase I-II feasibility study. Ann Oncol 2004;15:638-645.

112. Loughrey M, Trivett M, Lade S, et al. Diagnostic application of Epstein-Barr virus-encoded RNA in situ hybridisation. Pathology 2004;36(4):301-308.

113. Yap YY, Hassan S, Chan M, et al. Epstein-Barr virus DNA detection in the diagnosis of nasopharyngeal carcinoma. Otolaryngol Head Neck Surg 2007;136(6):986-991.

\section{Zhang MQ El-Mofty SK, Dávila RM. Detection of human} papillomavirus-related squamous cell carcinoma cytologically and by in situ hybridization in fine-needle aspiration biopsies of cervical metastasis: A tool for identifying the site of an occult head and neck primary. Cancer 2008;114(2):118-123.

115. Forastiere AA, Goepfert $\mathrm{H}$, Maor M, et al. Concurrent chemotherapy and radiotherapy for organ preservation in advanced laryngeal cancer. N Engl J Med 2003;349:2091-2098.

116. Department of Veterans Affairs Laryngeal Cancer Study Group. Induction chemotherapy plus radiation compared with surgery plus radiation in patients with advanced laryngeal cancer. N Engl J Med 1991;324:1685-1690.

117. Cooper IS, Rowe JD, Newall J. Regional stage IV carcinoma of the nasopharynx treated by aggressive radiotherapy. Int J Radiat Oncol Biol Phys 1983;9:1737-1745.
118. Bailet JW, Mark RI, Abemayor E, et al. Nasopharynx carcinoma: Treatment results with primary radiation therapy. Laryngoscope 1992;102:965-972.

119. Johansen LV, Mestre M, Overgaard J. Carcinoma of the nasopharynx: Analysis of treatment results in 167 consecutively admitted patients. Head Neck 1992;14:200-207.

120. Sanguineti G, Geara F, Garden A, et al. Carcinoma of the nasopharynx treated by radiotherapy alone: Determinants of local and regional control. Int Radiat Oncol Biol Phys 1997;37:985-996.

121. Wang CC. Radiation Therapy for Head and Neck Neoplasms, 3rd ed. New York: Wiley-Liss 1997:274.

122. Mesic JB, Fletcher GH, Geopfert $\mathrm{H}$. Mega-voltage irradiation of epithelial tumors of the nasopharynx. Int J Radiat Oncol Biol Phys 1981;7:452.

123. Frezza G, Barbieri F, Emiliani $E$, et al. Patterns of failure in nasopharyngeal cancer treated with megavoltage irradiation. Radiother Oncol 1986;5:287-294.

124. Santos IA, Gonzalez CP, Dela Fuente I, et al. Impact of change in the treatment of nasopharyngeal carcinoma: An experience of 30 years. Radiother Oncol 1995;36:121-127.

125. Perez CA, DeVineni VR, Marcial-Vega V, et al. Carcinoma of the nasopharynx: Factors affecting prognosis. Int J Radiat Oncol Biol Phys 1992;23:271-280.

126. Hoppe RT, Goffinet DR, Bagshaw MA. Carcinoma of the nasopharynx: Eighteen years experience with megavoltage radiation therapy. Cancer 1976;37:2605-2612.

127. Al-Sarraf M, LeBlanc M, Giri PG, et al. Chemoradiotherapy versus radiotherapy in patients with advanced nasopharyngeal cancer: phase III randomized Intergroup study 0099. J Clin Oncol 1998;16:1310-1317.

128. Wee J, Tan EH, Tai BC, et al. Randomized trial of radiotherapy versus concurrent chemoradiotherapy followed by adjuvant 
chemotherapy in patients with American Joint Committee on Cancer/International Union Against Cancer Stage III and IV nasopharyngeal cancer of the endemic variety. J Clin Oncol 2005;23:6730-6738.

129. Leong SS, Wee J, Tay MH, et al. Paclitaxel, carboplatin, and gemcitabine in metastatic nasopharyngeal carcinoma: a Phase II trial using a triplet combination. Cancer 2005;103:569-575.

130. Chan AT, Hsu MM, Goh BC, et al. Multicenter, phase II study of cetuximab in combination with carboplatin in patients with recurrent or metastatic nasopharyngeal carcinoma. J Clin Oncol 2005;23:3568-3576.

131. Zhang L, Zhang Y, Huang PY, et al. Phase II clinical study of gemcitabine in the treatment of patients with advanced nasopharyngeal carcinoma after the failure of platinum-based chemotherapy. Cancer Chemother Pharmacol 2008;61(1):33-38. Epub 2007 Mar 20.

132. Ngan RK, Yiu HH, Lau WH, et al. Combination gemcitabine and cisplatin chemotherapy for metastatic or recurrent nasopharyngeal carcinoma: report of a phase II study. Ann Oncol 2002;13(8):1252-1258.

133. Ma BB, Tannock IF, Pond GR, et al. Chemotherapy with gemcitabine-containing regimens for locally recurrent or metastatic nasopharyngeal carcinoma. Cancer 2002;95(12):2516-2523.

134. Posner MR, Ervin TJ, Miller D, et al. Incidence of hypothyroidism following multimodality treatment for advanced squamous-cell cancer of the head and neck. Laryngoscope 1984;94:451-454.

135. Adelstein DJ, Li Y, Adams GL, et al. An intergroup phase III comparison of standard radiation therapy and two schedules of concurrent chemoradiotherapy in patients with unresectable squamous cell head and neck cancer. J Clin Oncol 2003:21:92-98.

136. Lo TCM, Wiley AL Jr., Ansfield FJ, et al. Combined radiation therapy and 5-fluorouracil for advanced squamous cell carcinoma of the oral cavity and oropharynx: A randomized study. Am J Roentgenol 1976;126:229-235.

137. Sanchiz F, Milla A, Torner J, et al. Single fraction per day versus two fractions per day vs. radiochemotherapy in the treatment of head and neck cancer. Int J Radiation Oncol Biol Phys 1990;19:1347-1350.

138. Browman GP, Cripps C, Hodson DI, et al. Placebo-controlled randomized trial of infusional fluorouracil during standard radiotherapy in locally advanced head and neck cancer. J Clin Oncol 1994;12:2648-2653.

139. Smid L, Lesnicar H, Zakotnik B, et al. Radiotherapy combined with simultaneous chemotherapy with mitomycin $\mathrm{C}$ and bleomycin for inoperable head and neck cancer: Preliminary report. Int J Radiat Oncol Biol Phys 1995;32:769-775.

140. Bachaud J-M, Cohen-Jonathan E, Alzieu C, et al. Combined postoperative radiotherapy and weekly cisplatin infusion for locally advanced head and neck carcinoma: Final report of a randomized trial. Int J Radiat Oncol Biol Phys 1996;36:999-1004.

141. Merlano M, Benasso M, Corvo R, et al. Five-year update of a randomized trial of alternating radiotherapy and chemotherapy compared with radiotherapy alone in treatment of unresectable squamous cell carcinoma of the head and neck. J Natl Cancer Inst 1996;88:583-589.

142. Brizel DM, Albers ME Fisher SR, et al. Hyperfractionated irradiation with or without concurrent chemotherapy for locally advanced head and neck cancer. N Engl J Med 1998;338:1798-1804.

143. Wendt TG, Grabenbauer GG, Rodel CM, et al. Simultaneous Radiochemotherapy versus radiotherapy alone in advanced head and neck cancer: A randomized multicenter study. J Clin Oncol 1998;16:1318-1324.

144. Jeremic B, Shibamoto $Y$, Milicic N, et al. Hyperfractionated radiation therapy with or without concurrent low-dose daily cisplatin in 
locally advanced squamous cell carcinoma of the head and neck: A prospective randomized trial. J Clin Oncol 2000;18:1458-1464.

145. Munro AJ. An overview of randomised controlled trials of adjuvant chemotherapy in head and neck cancer. Br J Cancer 1995;71:83-91.

146. El-Sayed S, Nelson N. Adjuvant and adjunctive chemotherapy in the management of squamous cell carcinoma of the head and neck region: A meta-analysis of prospective and randomized trials. J Clin Oncol 1996;14:838-847.

147. Pignon JP, Bourhis J, Domenge $C$, et al on behalf of the $\mathrm{MACH}-\mathrm{NC}$ Collaborative Group. Chemotherapy added to locoregional treatment for head and neck squamous-cell carcinoma: Three metaanalyses of updated individual data. Lancet 2000;355:949-955.

148. Bourhis J, Amand C, Pignon J-P. Update of MACH-NC (MetaAnalysis of Chemotherapy in Head \& Neck Cancer) database focused on concomitant chemoradiotherapy. J Clin Oncol (Meeting Abstracts) 2004;22:5505.

149. Pignon JP, le Maître A, Bourhis J; MACH-NC Collaborative Group. Meta-analyses of chemotherapy in head and neck cancer (MACH-NC): an update. Int J Radiat Oncol Biol Phys 2007;69(2 Suppl):S112-S114.

150. Bonner JA, Harari PM, Giralt J, et al. Radiotherapy plus cetuximab for squamous-cell carcinoma of the head and neck. N Engl J Med 2006;354(6):567-578.

151. Mell LK, Weichselbaum RR. More on cetuximab in head and neck cancer. N Engl J Med 2007;357(21):2201-2202; author reply 2202-2203.

152. Garden AS, Harris J, Vokes EE, et al. Preliminary results of Radiation Therapy Oncology Group 97-03: A randomized phase II trial of concurrent radiation and chemotherapy for advanced squamous cell carcinomas of the head and neck. J Clin Oncol 2004;22:2856-2864.

153. Garden AS, Harris J, Voke EE, et al. Results of Radiation Therapy Oncology Group 97-03-A randomized phase II trial of concurrent radiation and chemotherapy for advanced squamous cell carcinomas of the head and neck: Long-term results and late toxicities. Int J Radiat Oncol Biol Phys 2007;69:S140.

154. Gibson MK, Li Y, Murphy B, et al. Randomized phase III evaluation of cisplatin plus fluorouracil versus cisplatin plus paclitaxel in advanced head and neck cancer (E1395): an intergroup trial of the Eastern Cooperative Oncology Group. J Clin Oncol 2005;23:3562-3567.

155. Forastiere AA, Metch B, Schuller DE, et al. Randomized comparison of cisplatin plus fluorouracil and carboplatin plus fluorouracil vs. methotrexate in advanced squamous-cell carcinoma of the head and neck: A Southwest Oncology Group study. J Clin Oncol 1992;10:1245-1251.

156. Burtness B, Goldwasser MA, Flood W, et al. Phase III randomized trial of cisplatin plus placebo compared with cisplatin plus cetuximab in metastatic/recurrent head and neck cancer: An Eastern Cooperative Oncology Group Study. J Clin Oncol 2005;23:8646-8654.

157. Bourhis J, Rivera F, Mesia R, et al. Phase I/II study of cetuximab in combination with cisplatin or carboplatin and fluorouracil in patients with recurrent or metastatic squamous cell carcinoma of the head and neck. J Clin Oncol 2006;24(18):2866-2872.

158. Herbst RS, Bunn PA, Jr. Targeting the epidermal growth factor receptor in non-small cell lung cancer. Clin Cancer Res 2003;9:5813-5824.

159. Herbst RS, Arquette M, Shin DM, et al. Phase II multicenter study of the epidermal growth factor receptor antibody cetuximab and cisplatin for recurrent and refractory squamous cell carcinoma of the head and neck. J Clin Oncol 2005;23:5578-5587.

160. Baselga J, Trigo JM, Bourhis J, et al. Phase II multicenter study of the antiepidermal growth factor receptor monoclonal antibody cetuximab in combination with platinum-based chemotherapy in patients with platinum-refractory metastatic and/or recurrent squamous cell carcinoma of the head and neck. J Clin Oncol 2005;23:5568-5577. 
161. Vermorken JB, Trigo J, Hitt R, et al. Open-label, uncontrolled, multicenter phase II study to evaluate the efficacy and toxicity of cetuximab as a single agent in patients with recurrent and/or metastatic squamous cell carcinoma of the head and neck who failed to respond to platinum-based therapy. J Clin Oncol 2007;25(16):2171-2177.

162. Vermorken J, Mesia R, Vega $\mathrm{V}$, et al. Cetuximab extends survival of patients with recurrent or metastatic SCCHN when added to first line platinum based therapy - Results of a randomized phase III (Extreme) study [abstract]. ASCO Annual Meeting Proceedings. J Clin Oncol 2007;25:6091.

163. Kim ES, Kies MS, Glisson BS, et al. Final results of a phase II study of erlotinib, docetaxel and cisplatin in patients with recurrent/metastatic head and neck cancer. [abstract]. ASCO Annual Meeting Proceedings. J Clin Oncol 2007;25:6013.

164. Hitt R, Irigoyen A, Nuñez J, et al. Phase Il study of combination cetuximab and weekly paclitaxel in patients with metastatic/recurrent squamous cell carcinoma of head and neck (SCCHN): Spanish Head and Neck Cancer Group (TTCC) [abstract]. ASCO Annual Meeting Proceedings. J Clin Oncol 2007;25:6012.

165. Jacobs C, Lyman G, Velez-Garcia E, et al. A phase III randomized study comparing cisplatin and fluorouracil as single agents and in combination for advanced squamous cell carcinoma of the head and neck. J Clin Oncol 1992;10:257-263.

166. Browman GP, Cronin L. Standard chemotherapy in squamous cell head and neck cancer: What we have learned from randomized trials. Semin Oncol 1994;21:311-319.

167. Clavel M, Vermorken JB, Cognetti F, et al. Randomized comparison of cisplatin, methotrexate, bleomycin and vincristine (CABO) vs. cisplatin and 5-fluorouracil (CF) versus cisplatin in recurrent or metastatic squamous cell carcinoma of the head and neck. Ann Oncol 1994;5:521-526.

\section{Recommended Reading}

Colletier PJ, Garden AS, Morrison WH, et al. Postoperative radiation for squamous cell carcinoma metastatic to cervical lymph nodes from an unknown primary site: Outcomes and patterns of failure. Head Neck 1998;20:674-681.

Datta NR, Choudry AD. Twice a day versus once a day radiation therapy in head and neck cancer. Int J Radiat Oncol Biol Phys 1989;17:132.

Davidson BJ, Spiro RH, Patel S, et al. Cervical metastases of occult origin: The impact of combined modality therapy. Am J Surg 1994;168:395-399.

Greven KM, Keys JW Jr, Williams DW 3rd, et al. Occult primary tumors of the head and neck; lack of benefit from position emission tomography imaging with 2-[F-18] fluoro-2-deoxy-D-glucose. Cancer 1999;114-118.

Jungehulsing M, Scheidhauer K, Damm M, et al. 2[F]-fluoro-2-deoxy-Dglucose position emission tomography is a sensitive tool for the detection of occult primary cancer (carcinoma of unknown primary syndrome) with head and neck lymph node manifestation. Otolaryngol Head Neck Surg 2000;123:294-301.

Martin $\mathrm{H}$, Morfit HM. Cervical lymph node metastasis as the first symptom of cancer. Surg Gynecol Obstet 1944;78:133-159.

Mendenhall WM, Mancuso AA, Parsons JT, et al. Diagnostic evaluation of squamous cell carcinoma metastatic to cervical lymph nodes from an unknown head and neck primary site. Head Neck 1998;20:739-744.

Pignon JP, Bourhis J, Domenge C, et al on behalf of the MACH-NC Collaborative Group. Chemotherapy added to locoregional treatment for head and neck squamous-cell carcinoma: Three meta-analyses of updated individual data. Lancet 2000;355:949-955.

Randall DA, Johnstone PA, Foss RD, et al. Tonsillectomy in diagnosis of the unknown primary tumor of the head and neck. Otolaryngol Head Neck Surg 2000;122:52-55. 
Shanta V, Krishnamurthi S. Combined bleomycin and radiotherapy in oral cancer. Clin Radiol 1980;31:617-620.

Spaulding MD, Fisher SG, Wolf GT, et al. Cooperative laryngeal cancer study group: Tumor response, toxicity, and survival after neoadjuvant organ-preserving chemotherapy for advanced laryngeal carcinoma. J Clin Oncol 1994;12:1592-1599.

Talmi YP, Wolf GT, Hazuka M, et al. Unknown primary of the head and neck. J Laryngol Otol 1996;110:353-356.

Weissler MC, Melin S, Sailer SL, et al. Simultaneous chemoradiation in the treatment of advanced head and neck cancer. Arch Otolaryngol Head Neck Surg 1992;188:806-810.

Wolf GT, Fisher SG. Effectiveness of salvage neck dissection for advanced regional metastases when induction chemotherapy and radiation are used for organ preservation. Laryngoscope 1992;102:934-939.

Wolf GT, Hong WK, Fisher SF. Neoadjuvant chemotherapy for organ preservation: Current status. Proceedings of the 4th International Conference on Head and Neck Cancer 1996;4:89-97. 